\title{
Review \\ Phytochemical Composition and Biological Activities of Scorzonera Species
}

\author{
Karolina Lendzion $\left.{ }^{1}{ }^{(}\right)$, Agnieszka Gornowicz ${ }^{1, *} \mathbb{C}$, Krzysztof Bielawski $^{2}$ and Anna Bielawska ${ }^{1}$ \\ 1 Department of Biotechnology, Medical University of Bialystok, Kilinskiego 1, 15-089 Bialystok, Poland \\ karolina.lendzion@umb.edu.pl (K.L.); aniabiel@umb.edu.pl (A.B.) \\ 2 Department of Synthesis and Technology of Drugs, Medical University of Bialystok, Kilinskiego 1, \\ 15-089 Bialystok, Poland; kbiel@umb.edu.pl \\ * Correspondence: agnieszka.gornowicz@umb.edu.pl; Tel.: +48-85-748-5742
}

Citation: Lendzion, K.; Gornowicz, A.; Bielawski, K.; Bielawska, A. Phytochemical Composition and Biological Activities of Scorzonera Species. Int. J. Mol. Sci. 2021, 22, 5128 https://doi.org/10.3390/ijms22105128

Academic Editor: Toshio Morikawa

Received: 16 April 2021

Accepted: 10 May 2021

Published: 12 May 2021

Publisher's Note: MDPI stays neutral with regard to jurisdictional claims in published maps and institutional affiliations.

Copyright: (c) 2021 by the authors. Licensee MDPI, Basel, Switzerland. This article is an open access article distributed under the terms and conditions of the Creative Commons Attribution (CC BY) license (https:// creativecommons.org/licenses/by/ $4.0 /)$.

\begin{abstract}
The genus Scorzonera comprises nearly 200 species, naturally occurring in Europe, Asia, and northern parts of Africa. Plants belonging to the Scorzonera genus have been a significant part of folk medicine in Asia, especially China, Mongolia, and Turkey for centuries. Therefore, they have become the subject of research regarding their phytochemical composition and biological activity. The aim of this review is to present and assess the phytochemical composition, and bioactive potential of species within the genus Scorzonera. Studies have shown the presence of many bioactive compounds like triterpenoids, sesquiterpenoids, flavonoids, or caffeic acid and quinic acid derivatives in extracts obtained from aerial and subaerial parts of the plants. The antioxidant and cytotoxic properties have been evaluated, together with the mechanism of anti-inflammatory, analgesic, and hepatoprotective activity. Scorzonera species have also been investigated for their activity against several bacteria and fungi strains. Despite mild cytotoxicity against cancer cell lines in vitro, the bioactive properties in wound healing therapy and the treatment of microbial infections might, in perspective, be the starting point for the research on Scorzonera species as active agents in medical products designed for miscellaneous skin conditions.
\end{abstract}

Keywords: Scorzonera; biological activity; herbal medicine; phytochemical composition; Asteraceae

\section{Introduction}

Scorzonera L. is a genus in the Cichorieae tribe of the Asteraceae family. It is spread mostly in central and southern parts of Europe, Eurasia, and Africa in arid areas [1,2]. Numerous species are endemic to Anatolia (Turkey) [1,3-11], Mongolia [12-15], and China [16-18] The genus comprises approximately 180-190 species [19], including S. hispanica, whose roots are a valued vegetable, with the taste similar to asparagus [20], and S. tau-saghyz (a species of interest in terms of obtaining natural rubber) [21]. Several Scorzonera species are a source of feed for farming animals in arid regions [15]. Typically, plants within the Scorzonera genus are perennial herbs characterized by the presence of a caudex or tuber. Biennial plants or dwarf subshrubs are rare [19]. Plants within the genus Scorzonera are reported to contain flavonoids [12,13,22-24], phenolic acid derivatives $[8,13,25,26]$, triterpenoids [18,23,27-31], sesquiterpenoids [14,17,20,32-34], dihydroisocoumarins [7,35-37], and other bioactive compounds. Scorzonera species have been commonly used as medicinal plants in European and Asian herbal therapy for ages. In Turkey, they are known as hemostatic agents, as well as, when used externally, as plasters in the process of wound healing [38]. The plants are also present in folk medicine as a remedy for hypertension, atherosclerosis, or kidney dysfunction [39].

This review aims to present the phytochemical composition of Scorzonera species, including the compounds characteristic for the genus, as well as novel compounds, which have not been previously isolated from Asteraceae. Extraction methods have been briefly summarized as well. A summary of available data regarding the use of Scorzonera in folk 
medicine has also been included. Phytochemical composition and ethnopharmacological reports lead to the third part of this paper, the assessment of biological activities of natural products (extracts, fractions, and pure compounds) obtained from species within the genus Scorzonera. To our best knowledge, this is the first comprehensive review of the current findings in the field of phytochemistry and bioactivity of Scorzonera species. The assessment of biological activity in vitro and in vivo is the first step in the development of new plantderived products and those play a substantial role in healthcare [40]. Some novel natural medicines are under clinical trials [41], others have been approved in therapy [42,43]. In this paper, a summary of the results of in vitro and in vivo studies has been made, as well as an attempt to evaluate their significance and therapeutic potential.

The search strategy for this review involved browsing results for terms 'Scorzonera' and 'biological activity' or 'bioactive', 'Scorzonera' and 'phytochemistry' 'phytochemical composition' in the following databases: Reaxys, PubMed, and ScienceDirect. The search was limited to the years 2000-2021, with three studies published before the year 2000 included in this review [44-46].

\section{Scorzonera in Traditional Medicine}

Genera within the family Asteraceae have been present in folk medicine across Europe, Asia, and northern Africa. That includes species within the Scorzonera genus, which are a significant part of Turkish traditional medicine in the therapy of arteriosclerosis, kidney disorders, wounds, rheumatism, but also as antidiabetic, antihypertensive, and antinociceptive medications [5]. The leaves of S. latifolia (Fisch and Mey.) DC., applied topically, act as plaster and prevent nausea. Turkish folk medicine uses latex obtained from S. latifolia to treat infertility and as an anthelmintic and pain-relieving medication $[3,6,38,47]$. Roots of $S$. tomentosa L. are believed to have hemostatic properties when ingested [38]. Aerial parts of S. laciniata L. are known as antipyretic, antipyogenic, antiatherosclerotic, antidiabetic, antirheumatic, and blood pressure-lowering agents in folk therapy [39]. Turkish folk medicine uses S. phaeopappa Boiss., S. sosnowskyii Lipsch., and S. mirabilis Lipsch. for headaches. S. mollis Biela is used as a diuretic and against kidney stones [48]. In Algerian traditional medicine, S. undulata ssp. deliciosa is a part of the treatment of snake bites [49]. Mongolian folk remedies for various ailments include Scorzonera species as well. There are reports for the use of $S$. pseudodivaricata Lipsch. as antipyretic in viral and bacterial infections, anti-diarrheal and diuretic agents, as well as for the treatment of lung edema and diseases caused by parasite infections. Aerial and subaerial parts of S. divaricata Turcz. are used to treat ulcers and stomach tumors. [14]. Leaves and shoots of S. divaricata are also present in the folk medicine of India in the therapy of jaundice [50]. Traditional Chinese medicine uses S. mongolica Maxim. root to reduce fever and treat carbuncle mastitis, as well as an antineoplastic agent [31]. Roots of Scorzonera hispanica L., currently cultivated and eaten as a vegetable, were formerly used in European folk medicine as a mucolytic agent in pulmonary diseases, appetite stimulator, and to defeat a cold. [14,32]. Tibetan folk medicine has used S. austriaca Willd. for the treatment of carbuncle, inflammation, and fever $[17,28]$. Scorzonera radiata Fisch. is a Mongolian traditional remedy for bacterial and viral infection-induced fever, poisonous ulcers, and as a lactation-inducing and diuretic agent [15]. In Libya, Scorzonera resedifolia L. is known as a folk medication for liver pain [51].

\section{Phytochemical Composition of Scorzonera Species}

Species within the Scorzonera genus are a source of flavonoid aglycones and glycosides, phenolic acids and their derivatives, lignans, triterpenoids, sesquiterpenoids, dihydroisocoumarins, bibenzyl derivatives, as well other compounds $[4,10,14,27,35,36,52-55]$.

\subsection{Scorzonera acuminata Boiss.}

The samples of Scorzonera acuminata aerial parts and roots were collected in the northern part of Anatolia, Turkey. In the study by Süntar et al. [9], plant samples were extracted with 
$20 \%$ aqueous methanol. The study by Bahadır-Acıkara et al. [27] includes a phytochemical analysis of $n$-hexane extracts of S. acuminata aerial parts and roots.

A $20 \%$ aqueous methanol extract of the aerial parts of S. acuminata was reported to contain chlorogenic acid, rutin, and cyranoside [9]. Another study reports the presence of $\alpha$-amyrin, lupeol, and lupeol acetate in the $n$-hexane extract [27].

In the aqueous methanol extract from the roots, chlorogenic acid and trace amounts of rutin were found [9]. An $n$-hexane extract contained lupeol, lupeol acetate, and $\alpha$-amyrin [27].

\subsection{Scorzonera aristata Rameond ex DC.}

Samples of $S$. aristata were collected in Northern Italy $[26,55,56]$. The leaves from the specimen in the study by Jehle et al. [56] were extracted with methanol and subsequently with a mixture of methanol, acetone and water $(3 / 1 / 1, v / v / v)$, the roots were first treated with the mixture of methanol, acetone, and water $(3 / 1 / 1, v / v / v)$, then were extracted with methanol. The subaerial parts of the samples in the study by Zidorn et al. [55] were mixed with a stock solution and sonicated with methanol, then HPLC was performed. A study by Granica and colleagues [26] involved extraction of aerial parts of the species with 50\% aqueous methanol and the HPLC analysis of obtained extracts.

From the aerial part extracts of the plant, flavonoids (rutin, isoorientin, and quercetin 3-O-glucoside) and caffeic acid derivatives (chlorogenic acid, 3,5-dicaffeoylquinic acid, 4,5-dicaffeoylquinic acid) were isolated [56]. In the 50\% aqueous methanol extract, the presence of chlorogenic acid, 4-O-caffeoylquinic acid, 1,5-O-dicaffeoylquinic acid, and rutin, as well as apigenin derivative and luteolin derivative [26].

From subaerial parts, 3,5-dicaffeoylquinic acid and caffeic acid methyl ester were isolated, along with the following triterpenes: lupeol, magnificol, and $3 \alpha$-hydroxyolean-5ene [56]. The presence of chlorogenic acid and 3,5-dicaffeoylquinic acid was previously reported in the study by Zidorn et al. [55].

\subsection{Scorzonera aucheriana DC.}

Samples of S. aucheriana were collected in central Turkey and aerial parts were extracted with methanol at room temperature [7,29].

Investigation on aerial parts of the plant led to the isolation of dihydroisocoumarins and dihydroisocoumarin derivatives (scorzopygmaecoside, scorzocreticoside II, isoscorzopygmaecoside, scorzoaucherioside I and II), quinic acid derivatives (3,5-O-dicaffeoylepi-quinic acid and 3,5-O-dicaffeoylquinic acid), and 3,4-dihydroxyphenyl caffeate [7]. In another study, chlorogenic acid derivatives (methyl 1-(2-methylcyclopropyl-1-carbonyloxy) chlorogenate and 3,4-bis[(3',4-dioxo-1', 3', 5', 6' -tetrahydrospiro[cyclohexa-2,5-diene-1, $4^{\prime}$ cyclopenta[c]-furan]-1'-yl)]chlorogenic acid), triterpenoids (taraxasterol, taraxasterol acetate, taraxasterol oleate, lupeol, lupeol acetate and ptiloepoxide) and $\beta$-sitosterol were isolated from the methanol extract of S. aucheriana aerial parts [29].

\subsection{Scorzonera austriaca Willd.}

Samples of S. austriaca were collected in the northeast [24] and central [17,33] parts of China.

Herbs of S. austriaca were extracted with $70 \%$ aqueous ethanol. The extract was reported to contain the following flavonoid glycosides and flavonoid glycoside derivatives: $5,7,4^{\prime}$ trihydroxyflavone 6-C-(2'I-O- $\beta$-D-glucopyranosyl $\beta$-D-glucopyranoside), 5,7,3' $4^{\prime}$-tetrahydroxyflavone 6-C-(2" -O- $\beta$-D-glucopyranosyl $\beta$-D-glucopyranoside), quercetin 3-O-rutinoside, $5,7,4^{\prime}$-trihydroxyflavone 6-C- $\beta$-D-glucopyranoside, $3^{\prime}$-methoxy-5,7, $4^{\prime}$-trihydroxyflavone $6-C$ $\beta$-D-glucopyranoside, 5,7,4'-trihydroxyflavone 8-C-(6" ${ }^{\prime \prime} \mathrm{O}$-trans-caffeoyl $\beta$-D-glucopyranoside and 5,7,3', $4^{\prime}$-tetrahydroxyflavone $8-C-\left(6^{\prime \prime}\right.$-O-trans-caffeoyl $\beta$-D-glucopyranoside [24].

Roots of the plant samples collected in central China were extracted with acetone and guaianolides (biguaiascorzolide A and biguaiascorzolide B) were isolated [17]. Before that discovery, in 2004, Li et al. [16] isolated a sesquiterpene lactone ( $3 \beta, 11 \alpha$-dihydroxy- $4 \beta$-methylguaia-10 (14)-en-12, $6 \alpha$-olide) from the acetone extract of $S$. austriaca roots. Other identified 
in the root acetone extract sesquiterpenoids were: scorzoaustriacoside, scorzoaustriacin, scorzoaustriacin 3-O- $\beta$-D-glucoside, 4-epi-dihydroestafiatol, 14-isovaleroxyscorzoaustricin, 14-isovaleroxyscorzoaustricin sulfate, zaluzanin $C$, glucozaluzanic $C$, dehydrocostus lactone, 11 $\beta, 13$-dihydrozaluzanin and diacetoxyisolippidiol [33]. In the study by Wu et al. from 2011 [28], the following compounds were isolated from the acetone root extract: oleanane-type triterpenes ( $3 \beta$-acetoxyglutin-5(10)-en-6-oxo, glutinol, $\beta$-amyrin-3-( $3^{\prime}$-methylbutanonate), $\beta$-amyrin 3-acetyl, $3 \beta$-acetyl-11 $\alpha, 12 \alpha$-oxidotaraxerol), ursane-type triterpenes ( $\alpha$-amyrin 3acetyl, $\alpha$-amyrin 3-acetyl-11-oxo, D-friedours-14-en-3 $\beta$-acetyl-11 $\alpha, 12 \alpha$-epoxy, taraxasterol, and $\psi$-taraxasteryl 3(3'-methyl-butanonate)), lupeol, (23Z)-cycloart-23-ene-3 $\beta$, 25-dihydroxy, 9 $\beta, 19$-cyclolanostane-24-en-3-oxo, and steroids ( $\beta$-sitosterol, $\beta$-stigmasterol, stigmast-4-en-3one, stigmast- $3 \beta, 5 \alpha, 6 \beta$-trihydroxy, and $\beta$-sitosterol 3 - $\beta$-D-glucoside).

\subsection{Scorzonera baetica (Boiss.) Boiss.}

Aerial parts of plant samples, collected in Spain, were extracted with $50 \%$ aqueous methanol at room temperature and the extracts were analyzed using HPLC.

The aerial part $50 \%$ methanol extract was reported to contain caffeoylquinic acid derivatives (3-O-caffeoylquinic acid, 4-O-caffeoylquinic acid, chlorogenic acid, 1,5-O-dicaffeoylquinic acid, 3,5-O-dicaffeoylquinic acid, 4,5-O-dicaffeoylquinic acid), flavonoid glycosides (orientin, isoorientin, vitexin, isovitexin, cyranoside), and flavonoid diglycosides [26].

\subsection{Scorzonera cana (C.A. Meyer) Hoffm. var. alpina (Boiss.) Chamb.}

Plant samples were collected in the north-central part of Turkey and extracted with $20 \%$ aqueous methanol at room temperature.

In the extract of the aerial parts, rutin and notable amounts of chlorogenic acid were reported present.

The presence of chlorogenic acid was detected in the root extract [9].

\subsection{Scorzonera cana (C.A. Meyer) Hoffm. var. jacquiniana (W. Koch) Chamb.}

Plant samples were collected in central Turkey $[9,27]$. Aerial and subaerial parts were separated and extracted with $n$-hexane [27] and 20\% aqueous methanol [9].

Triterpenoids present in the $n$-hexane extract of the aerial parts were taraxasteryl acetate, lupeol, lupeol acetate, and $\alpha$-amyrin [27]. Compounds present in the aqueous methanol extract were: chlorogenic acid, rutin, hyperoside, luteolin 7-glucoside, and trace amounts of apigenin [9].

In the root aqueous methanol extracts, chlorogenic acid was found. [9]. There were also reports on the presence of $\alpha$-amyrin. taraxasteryl acetate, lupeol, and lupeol acetate in the $n$-hexane extract [27].

\subsection{Scorzonera cinerea Boiss.}

Samples of $S$. cinerea collected in central Turkey were extracted with $n$-hexane at room temperature.

In the extract of the aerial parts of $S$. cinerea, the following triterpenoids were detected: lupeol, lupeol acetate, taraxasteryl acetate, $3 \beta$-hydroxy-fern-7-en-6-one-acetate, $\alpha$-amyrin, and olean-12-en-11-one-3-acetyl.

In the root extract of the plant, taraxasteryl acetate, lupeol, lupeol acetate, and $\alpha-$ amyrin were detected [27].

\subsection{Scorzonera cretica Willd.}

Samples of S. cretica were collected on Crete, Greece. The whole plant was subjected to extraction with dichloromethane and subsequently with methanol.

From the dichloromethane extract, dihydroisocoumarin (scorzocreticin), dihydroisocoumarins glycosides (scorzocreticoside I, scorzocreticoside II), and 3-O- $\beta$-D-glucopyranosylsitosterol were isolated. Triterpenoids (lupeol, lupeol acetate, lupenone, germanicol, german- 
icol acetate, germanicone, taraxasterol, taraxasterol acetate, oleanol, oleanol acetate) were isolated from the methanol extract of the plant [37].

\subsection{Scorzonera crispatula Boiss.}

Plant samples (aerial parts) were collected in England [44] and Spain. Samples from Spain were extracted at room temperature with a mixture of methanol and water $(1: 1, v / v)$ and subjected to HPLC [26]. Samples collected in England were extracted with ethanol [44].

In the $S$. crispatula aerial part hydromethanolic extract, the presence of caffeic acid derivatives (3-O-caffeoylquinic acid, 4-O-caffeoylquinic acid, chlorogenic acid, 1,5-O-dicaffeoylquinic acid. 3,5-O-dicaffeoylquinic acid and 4,5-O-dicaffeoylquinic acid), flavonoid aglycones (quercetin and luteolin) flavonoid C-glycosides (isoorientin and isovitexin), and several flavonoid diglycosides was reported [26]. In the ethanol extract of S. crispatula, luteolin and quercetin were detected.

\subsection{Scorzonera divaricata Turcz.}

Samples of S. divaricata were collected in Mongolia [14] and central China [34,57-59]. Aerial parts were extracted with methanol at room temperature [14,34], then with methanol at $65^{\circ} \mathrm{C}$ [34]. Subaerial parts were subjected to extraction with 95\% aqueous ethanol [57-59].

From the aerial part methanol extract, feruloylpodospermic acid A and feruloylpodospermic acid $B$ were isolated and the presence of known compounds (scopoletin, chlorogenic acid, isovitexin 4'-O-glucoside, isovitexin 2'-O-xyloside, kaempferol 3-O-rutinoside, apigenin) was detected [14]. The aerial part methanol extract was used to isolate the novel compounds sulfoscorzonin D and sulfoscorzonin E. Apart from sulfoscorzonins, the following compounds were isolated: benzoic acid derivatives (methyl-3,4-dihydroxybenzoate, $m$-hydroxybenzoic acid), coumarin derivatives (scopoletin, 7-hydroxycoumarin), flavonoid aglycones (diosmetin, luteolin, tricin, 7,3' , 4'-trihydroxyflavonol, 5,7-dihydroxy-8-methoxyflavone, 5,7-dihydroxy-6methoxyflavone), phenolic acid derivatives (trans-caffeic acid, trans-p-hydroxycoumaric acid, 4-hydroxy-3-methoxyphenyl ferulate), sesquiterpenoids (glucozaluzanin $C, 1 \beta, 4 \alpha$-dihydroxy$5 \alpha, 6 \beta, 7 \alpha, 11 \beta H$-eudermn-12,6-olide), steroids ((22E)-5 $\alpha, 8 \alpha$-epidioxyergosta-6,22-dien-3 $\beta$-ol, ergosta-3 $\beta, 5 \alpha, 6 \beta$-trialcohol, stigma-5-en-3-O- $\beta$-glucoside), triterpenoids (oleanolic acid and lup-20(29)-ene-3 $\beta, 28-$ diol), sacrolide A, and vomifoliol [34].

From the subaerial part ethanolic extract, the following compounds were isolated: phenolic acids and their derivatives ((-)-1,4-di-O-feruloyl-3-O-dihydrocaffeoylquinic acid, (-)-1-O-feruloyl-4-O-dihydrocaffeoylquinic acid (-)-3,5-di-O-feruloylquinic acid, (-)-1-Oferuloyl-3-O-dihydrocaffeoylquinic acid, (-)-1-O-feruloyl-5-O-dihydrocaffeoylquinic acid, 3-O-feruloylquinate, butyl 3-O-feruloylquinate, caffeic acid, dihydrocaffeic acid and its methyl, ethyl and n-butyl esters), triterpenoids (scorzodivaricin A, scorzodivaricin B and scorzodivaricin C, scorzodivaricin D, 23(Z)-3 $\beta$-acetoxy-25-hydroxy-tirucalla-7,23-diene, 23(Z)-3 $\beta, 25$-dihydroxy-tirucalla-7,23-diene, 23(Z)-3 $\beta, 25$-dihydroxy-tirucalla-7,23-diene, 20(R)-3 $\beta$-acetoxy21-hydroxy-24(31)-methylene-dammarane and oleanolic acid), sesquiterpenoids (sulfoscorzonin A, sulfoscorzonin B, sulfoscorzonin C, and 10(Z)-1-oxo-bisabola2,10-dien-13-al), steroids ( $5 \alpha, 8 \alpha$-epidioxy-(22E,24R)-ergosta-6,22-dien-3 $\beta$-ol, stigmast-4en-6 $\beta$-ol-3-one $6 \beta$-hydroxystigmastan-4-en-3-one, $3 \beta$-hydroxystigmast-5-en-7-one, 5,6 $\alpha$ epoxy- $5 \alpha$-stigmastan-3 $\beta$-ol, $7 \beta$-hydroxysitosterol, $7 \alpha$-hydroxysitosterol, $\beta$-sitosterol, and $\beta$-daucosterol), benzene derivatives (vanillin, vanillic acid $4-O-\beta$-D-glucoside, vanillic acid 1-O- $\beta$-D-glucopyranosyl ester, tachioside, syringic acid ethyl ester, and 3,4-dimenthoxy3'-hydroxy propiophenone), fatty acids (pinellic acid, linoleic acid, and palmitic acid), coumarin derivatives (scopolin and scopoletin), and a lignan (pinoresinol) [57-59].

\subsection{Scorzonera eriophora DC.}

Samples of S. eriophora aerial and subaerial parts were collected in Turkey and extracted at room temperature with $20 \%$ aqueous methanol [9] and $n$-hexane [27].

Chlorogenic acid was detected in aqueous methanol extracts of both aerial parts and roots. The aerial part extract was also reported to contain luteolin and luteolin 7 - 
glycoside [9]. The $n$-hexane extracts of aerial and subaerial parts both contained taraxasteryl acetate, lupeol, lupeol acetate Additionally, $3 \beta$-hydroxy-fern-7-en-6-one-acetate was reported to be present in the $n$-hexane root extract [27].

\subsection{Scorzonera graminifolia $L$.}

Aerial parts of the plant, collected in England, were extracted with ethanol and reported to contain quercetin and luteolin [44].

\subsection{Scorzonera hieraciifolia Hayek}

Samples of the plant were collected in central Turkey. Aerial and subaerial parts were separated, and aerial parts were extracted with ethanol at room temperature and fractioned. Then isolation of compounds was performed.

From the subaerial part ethanol extract, the following compounds were isolated: quinic acid derivatives (5-O-feruloyl quinic acid methyl ester, 1,5-di-O-feruloylquinic acid, chlorogenic acid methyl ester, 3-O-caffeoylquinic acid methyl ester, 1,3-di-O-caffeoylquinic acid methyl ester, 3,5-di-O-caffeoylquinic acid methyl ester, and 4,5-di-O-caffeoylquinic acid methyl ester), caffeic acid, and 3-(4'-hydroxyphenyl)-2-propenoic acid (4" ${ }^{\prime \prime}$-carboxyl)phenyl ester [8].

\subsection{Scorzonera hirsuta L.}

Samples of S. hirsuta aerial parts, collected from the University of Reading (Reading, UK), were extracted with alcohol and the extract was reported to contain flavonoid aglycones: kaempferol, luteolin, and quercetin [44].

\subsection{Scorzonera hispanica $L$.}

In the studies by Granica et al. [20,26], plant samples were collected in Germany, subaerial parts for the quantitative analyses were purchased in Austria and Warsaw. In the study by Zidorn et al. [32], plant samples from Belgium were used. In the study by Petkova [60], the plant was harvested in Bulgaria.

For the isolation and identification of major constituents of S. hispanica subaerial parts, maceration with ethyl acetate was carried out. The phenolic compounds in aerial and subaerial parts were quantified using a modification of a method described by Zidorn et al. [61] with a mixture of methanol/acetone/water (3:1:1) [20]. For the elucidation of inulin content, the roots were extracted with water via microwave-assisted extraction [60].

In the aerial part aqueous methanol extract, the following compounds were detected: flavonoid glycosides (isoorientin, hyperoside, isoquercitrin, miquelianin), luteolin di-Cglycoside ( $C$-hexoside, $C$-pentoside), quercetin, caffeic acid, and caffeic acid derivatives (chlorogenic acid, 4-O-caffeoylquinic acid 1,5-O-dicaffeoylquinic acid, 3,5-O-dicaffeoylquinic acid, 4,5-O-dicaffeoylquinic acid, 3-O-caffeoylquinic acid, 4-O-caffeoylquinic acid, 1,5-Odicaffeoylquinic acid, 3,5-O-dicafeoylquinic acid, 4,5-O-dicaffeoylquinic acid) [20,26].

From the methanol extract of $S$. hispanica subaerial parts, plugitone, ixerioside $D$, and 3-O-angeloyl-11 $\beta, 13$-dihydrodesacylcynaropicrin-8 $\beta$-D-glucoside were isolated [32].

Subaerial part ethyl acetate extract was reported to contain a lignan ((-)-syringaresinol), octadecadienoic acids (linoleic acid, 9-hydroxyocta-(10E,12E)-decadienoic acid, 8-13-oxo(9Z,11E)-octadecadienoic acid, 9-oxo-(10E,12Z)-octadecadienoic acid, 13-oxo-(9E,11E)-octadecadienoic acid, and 9-oxo-(10E,12E)-octadecadienoic acid), and sesquiterpenoids (1-oxo-bisabola(2,10E)-diene-12-carboxylic acid, 1-oxo-bisabola-(2,10E)-diene-12-ol, plitostemonol, puliglutone, 1-oxo-bisabola-(2,10E)-diene-12-carboxylic acid methyl ester, 2,9-epoxycurcumen-12-al, and ixerisoside D) [20].

Caffeic acid and caffeic acid derivatives (chlorogenic acid, 4-O-caffeoylquinic acid, 1,5-O-dicaffeoylquinic acid, 3,5-O-dicaffeoylquinic acid, and 4,5-O-dicaffeoylquinic acid) were identified in subaerial part samples as well [20]. Notable amounts of inulin (over 20\% of dry plant material) were identified in the roots of S. hispanica [60]. 


\subsection{Scorzonera humilis L.}

Samples of S. humilis were collected in Austria and subaerial parts of the plant were extracted with methanol [53-55].

From the methanol extract, tyrolobibenzyls were isolated via column chromatography [53-55]. Tyrolobibenzyls A, B, and C were isolated and identified in the study from 2000, together with a lignin - pinoresinol-1-yl $\beta$-D-glucopyranoside [54]. In the study from 2002, the structure of tyrolobibenzyl D was elucidated [53]. A year later, the structure of two novel tyrolobibenzyls ( $\mathrm{E}$ and $\mathrm{F}$ ) was identified and the presence of chlorogenic acid and 3,5-dicaffeoylquinic acid was detected [55].

\subsection{Scorzonera incisa DC.}

Samples of the plant were collected in Turkey and aerial and subaerial parts were extracted separately at room temperature with $20 \%$ aqueous methanol [5] and $n$-hexane [27].

The aerial part $n$-hexane extract was reported to contain triterpenes: lupeol, lupeol acetate, $\alpha$-amyrin, and taraxasteryl acetate [27]. Additionally, the presence of rutin, cyranoside, and chlorogenic acid was detected in the aqueous methanol extract [5].

In the root extracts, the presence of chlorogenic acid was detected in the aqueous methanol extract [5]. Triterpenoids (lupeol and lupeol acetate, taraxasteryl acetate, and olean-12-en-11-one-3-acetyl) were reported present in the $n$-hexane root extract $[5,27]$.

\subsection{Scorzonera judaica Eig.}

The roots of the plant were collected in Jordan and subsequently extracted with $n$ hexane, chloroform, a mixture of chloroform and methanol (9:1), and methanol. Then, the isolation of compounds was carried out.

From the chloroform root extract, $4 \alpha$-hydroxypinoresinol, hydrangenol, and scorzotomentosin were isolated.

The $\mathrm{CHCl}_{3}: \mathrm{MeOH}(9: 1)$ extract was reported to contain 3S-hydrangenol $4^{\prime}-\mathrm{O}-\alpha-\mathrm{L}-$ rhamnopyranosyl-( $1 \rightarrow 3)-\beta$-D-glucopyranoside, hydrangenol 4 '-O- $\beta$-D-apiofuranosyl-( $1 \rightarrow 6)$ $\beta$-D-glucopyranoside, 2-hydroxy-6-[2-(4-hydroxyphenyl)-2-oxo-ethyl]benzoic acid, $E$-3-(3,4dihydroxybenzylidene)-5-(3,4-dihydroxyphenyl)dihydrofuran-2-one, Z-3-(3,4-dihydroxybenzylidene)-5-(3,4-dihydroxyphenyl)-2(3H)-furanone, $\quad 4$-[ $\beta$-D-glucopyranosyl)hydroxy]pinoresinol, hydrangenol 8-O- $\beta$-D-glucopyranoside, hydramacrophyllol A, hydramacrophyllol B, $4 \alpha$-hydroxypinoresinol, hydrangenol 4'-O- $\beta$-D-glucopyranoside, thunberginol F, and hydrangenol.

From the methanol extract, the following compounds were isolated: 3S-hydrangenol 4'$O$ - $\alpha$-L-rhamnopyranosyl-( $1 \rightarrow 3)-\beta$-D-glucopyranoside, hydrangenol 4 ' $-O-\beta$-D-apiofuranosyl$(1 \rightarrow 6)-\beta$-D-glucopyranoside, thunberginol F 7-O- $\beta$-D-glucopyranoside, 2-hydroxy-6-[2-(4hydroxyphenyl)-2-oxo-ethyl]benzoic acid, 2-hydroxy-6-[2-(3,4-dihydroxyphenyl)-2-oxo-ethyl] benzoic acid, 2-hydroxy-6-[2-(3,4-dihydroxyphenyl-5-methoxy)-2-oxoethyl]benzoic acid, hydrangeic acid 4'-O- $\beta$-D-glucopyranoside, 4-[ $\beta$-D-glucopyranosyl)hydroxy]-pinoresinol, hydrangenol 8-O- $\beta$-D-glucopyranoside, and hydrangenol 4'-O- $\beta$-D-glucopyranoside [25].

\subsection{Scorzonera laciniata L. ssp. laciniata}

Plant samples were collected in the north-western [9,27] and eastern parts of Turkey. [22]. Aerial and subaerial parts were separated and extracted with 20\% aqueous methanol [9] and $n$-hexane [27]. In the study by Erden et al. [22], the extraction was carried out using methanol, a mixture of hexane and isopropyl alcohol $(3: 2, v / v)$, water, and a mixture of $\mathrm{HNO}_{3}: \mathrm{H}_{2} \mathrm{SO}_{4}: \mathrm{H}_{2} \mathrm{O}_{2}(10: 1: 1, v / v / v)$.

In the aerial part $20 \%$ aqueous methanol extract, chlorogenic acid and luteolin 7glucoside, as well as trace amounts of rutin were reported [9]. Myricetin, kaempferol, and trace amounts of morin and quercetin were present in the methanol extract. Phytosterols (ergosterol, stigmasterol, and $\beta$-sitosterol) and vitamins $\mathrm{D}$ and $\mathrm{K}$ were identified in the hexane/isopropyl alcohol $(3: 2, v / v)$ extract and notable amounts of potassium were identified in the extract obtained with the mixture of $\mathrm{HNO}_{3}: \mathrm{H}_{2} \mathrm{SO}_{4}: \mathrm{H}_{2} \mathrm{O}_{2}$ [22]. The presence of 
lupeol, lupeol acetate, taraxasteryl acetate, and $\alpha$-amyrin in the $n$-hexane aerial part extract has been reported as well [27].

Root aqueous methanol extract contains chlorogenic acid [9]. In the $n$-hexane extract of the roots, triterpenoids (taraxasteryl acetate, lupeol, lupeol acetate, and $\alpha$-amyrin) were detected [27].

\subsection{Scorzonera latifolia (Fisch. and Mey.) DC.}

Samples of S. latifolia were collected in eastern $[5,27]$ and central-eastern Turkey $[10,22]$. Aerial and subaerial parts were separated and extracted with $20 \%$ aqueous methanol [5], and $n$-hexane [27]. The study by Erden et al. [22] reports extraction with methanol, a mixture of hexane and isopropyl alcohol $(3: 2, v / v)$, water, and a mixture of $\mathrm{HNO}_{3}: \mathrm{H}_{2} \mathrm{SO}_{4}: \mathrm{H}_{2} \mathrm{O}_{2}$ $(10: 1: 1, v / v / v)$.

Aerial part methanol extract of S. latifolia was reported to contain flavonoid aglycones: myricetin, quercetin, kaempferol, and morin. Ergosterol, stigmasterol, and $\beta$-sitosterol were also reported present in the hexane:isopropyl alcohol $(3: 2, v / v)$ extract from the aerial parts of the plant, together with retinol and vitamins D, E, and K [22]. Triterpenoids identified in aerial part $n$-hexane extract were: taraxasteryl acetate, $3 \beta$-hydroxy-fern- 7 -en6-one-acetate, lupeol, lupeol acetate, and $\alpha$-amyrin [27]. The aqueous methanol extract from the aerial parts was reported to contain chlorogenic acid, hyperoside, and luteolin 7-glucoside [5].

In the roots, the following triterpenoids are reported to be present in the $n$-hexane extract: taraxasteryl acetate, lupeol, lupeol acetate, $\beta$-hydroxy-fern-7-en-6-one-acetate, and olean-12-en-11-one-3-acetyl [27]. Moreover, chlorogenic acid was detected in the 20\% aqueous methanol extract [5].

\subsection{Scorzonera mirabilis Lipsch.}

Samples of the plant were collected in the city of Van, eastern Turkey, aerial parts and roots were separated in extracted at room temperature with $n$-hexane.

Both aerial and subaerial part $n$-hexane extracts of $S$. mirabilis contain taraxasteryl acetate, lupeol, and lupeol acetate [27].

\subsection{Scorzonera mollis Bieb. ssp. szowitsii (DC.) Chamb.}

Plants samples were collected in north-central Turkey [5,27]. Aerial and subaerial parts were separated and extracted at room temperature using $n$-hexane [27] and 20\% aqueous methanol [5].

In the aqueous methanol extract of aerial parts, chlorogenic acid, rutin, hyperoside, and cyranoside were detected [5]. The presence of taraxasteryl acetate, lupeol, lupeol acetate, and $\alpha$-amyrin was reported in $n$-hexane extracts from both aerial and subaerial parts of the plant [27].

Aqueous methanol root extract was reported to contain chlorogenic acid [5].

\subsection{Scorzonera papposa DC.}

Samples were collected in Jordan. Aerial parts and roots were separated, dried, and sequentially macerated with $n$-hexane, chloroform, a mixture of chloroform and methanol (9:1), and methanol.

From the aerial part methanol extract, the following compounds were isolated: (6trans-p-coumaroyl)-3-O- $\beta$-D-glucopyranosyl-2-deoxy-D-riburonic acid, a mixture of (6-cis- $p$ coumaroyl)-3-O- $\beta$-D-glucopyranosyl-2-deoxy-D-riburonic acid and (6-cis- $p$-coumaroyl)-3-O$\beta$-D-glucopyranosyl-2-deoxy-D-ribono-c-lactone, (6-trans- $p$-coumaroyl)-3-O- $\beta$-D-glucopyranosyl-2-deoxy-D-riburonic acid methyl ester, (6-trans- $p$-coumaroyl)-3-O- $\beta$-D-glucopyranosyl-(5-acetyl)-2-deoxy-D-riburonic acid, isoorientin, orientin, isoschaftoside, and swertiajaponin. From the $\mathrm{CHCl}_{3}: \mathrm{MeOH}$ extract, (6-trans- $p$-coumaroyl)-3-O- $\beta$-D-glucopyranosyl-2deoxy-D-riburonic acid, a mixture of (6-cis- $p$-coumaroyl)-3-O- $\beta$-D-glucopyranosyl-2-deoxyD-riburonic acid and (6-cis- $p$-coumaroyl)-3-O- $\beta$-D-glucopyranosyl-2-deoxy-D-ribono-c-lac- 
tone, and (6-trans-p-coumaroyl)-3-O- $\beta$-D-glucopyranosyl-(5-acetyl)-2-deoxy-D-riburonic acid were isolated.

The root methanol extract was reported to contain thunberginol G [62].

\subsection{Scorzonera parviflora Jacq.}

Aerial and subaerial parts of the plant, collected in central Turkey, were separated and extracted at room temperature using $n$-hexane [27] and 20\% aqueous methanol [5].

Aerial part $n$-hexane extract of $S$. parviflora was reported to contain the following triterpenoids: taraxasteryl acetate, lupeol, and lupeol acetate [27]. The aqueous methanol extract contained chlorogenic acid, hyperoside, and cynaroside [5].

In the roots, chlorogenic acid was detected in the aqueous methanol extract [5]. Taraxasteryl acetate, lupeol, and lupeol acetate were the main components of the $n$-hexane extract [27].

\subsection{Scorzonera pseudodivaricata Lipsch.}

Samples for the analysis were collected in Mongolia. Aerial and subaerial parts of the plant were separated, then aerial parts were macerated with methanol at room temperature.

Aerial part extract of the plant is reported to contain isochlorogenic acid A, cynaroside, isovitexin $2^{\prime \prime}$-O-xyloside, luteolin, luteolin 5-O-glucoside, platyphylloside, scopoletin, scorzoneric acid and scorzonerin [14].

\subsection{Scorzonera pusilla Pall.}

In the aerial parts, collected in Reading (England), the presence of quercetin and luteolin was reported [44].

\subsection{Scorzonera pygmaea Sibth. and Sm.}

Plant samples (subaerial parts) were collected in Turkey. The dried and powdered subaerial parts were macerated in ethanol.

From the subaerial part methanol extract, the following compounds were isolated: 3,5-dicaffeoylquinic acid, chlorogenic acid, chlorogenic acid methyl ester, cudrabibenzyl A, scorzocreticoside I scorzocreticoside II, scorzonerol, scorzopygmaecoside, and thunberginol C [35].

\subsection{Scorzonera radiata Fisch.}

Samples of S. radiata aerial parts were collected in Mongolia and macerated with methanol at room temperature $[12,13,15]$.

From the aerial part methanol extract of $S$. radiata, scorzodihydrostibenes A-E were isolated [15]. Apart from that, the presence of 3,5-dicaffeoyl-epi-quinic acid, 3,5-dicaffeoylquinic acid, 4,5-dicaffeoylquinic acid, 5-p-coumaroylquinic acid (cis and trans), chlorogenic acid, isoorientin, kaempferol 3-O-rutinoside, macroantoin $\mathrm{F}$, macroantoin $\mathrm{G}$, quinic acid, rutin, and violanthin was detected [12], Moreover, scorzonerin A, scorzonerin B, and 4,5-dicaffeoylepi-quinic acid were isolated [13].

\subsection{Scorzonera suberosa C. Koch ssp. suberosa}

Samples of the plant were collected in the central part of Turkey. Aerial and subaerial parts were separated and extracted with $n$-hexane at room temperature [27]. In the study by Erden et al. [22], the solvents used for extraction were methanol, a mixture of hexane and isopropyl alcohol $(3: 2, v / v)$, water, and a mixture of $\mathrm{HNO}_{3}: \mathrm{H}_{2} \mathrm{SO}_{4}: \mathrm{H}_{2} \mathrm{O}_{2}(10: 1: 1, v / v / v)$.

Aerial parts and roots are reported to contain taraxasteryl acetate, lupeol, and lupeol acetate [27]. Myricetin, morin, and quercetin were found in the methanol extract, vitamins $\mathrm{D}$, E, and K, retinol, and phytosterols: $\beta$-sitosterol, ergosterol, and stigmasterol were detected in the hexane/isopropyl alcohol extract. Sodium and potassium were also reported present (extraction using the mixture of $\mathrm{HNO}_{3}: \mathrm{H}_{2} \mathrm{SO}_{4}: \mathrm{H}_{2} \mathrm{O}_{2}$ ) [22]. 


\subsection{Scorzonera sublanata Lipsch.}

Samples of S. sublanata were collected in Turkey and aerial parts and roots were separated [9,27]. The extraction was carried out using $n$-hexane [27] and $20 \%$ aqueous methanol [9].

Aerial part aqueous methanol extract contains chlorogenic acid and hyperoside [9]. In the $n$-hexane extract, the presence of lupeol, lupeol acetate, and taraxasteryl acetate was reported [27].

Root aqueous methanol extract was reported to contain chlorogenic acid [9]. The $n$-hexane extract is reported to contain taraxasteryl acetate, $3 \beta$-hydroxy-fern-7-en-6-oneacetate, lupeol, and lupeol acetate [27].

\subsection{Scorzonera tomentosa $L$.}

Samples of $S$. tomentosa were collected in Turkey $[5,27,36]$. Aerial parts and roots were separated and extracted using $n$-hexane [27], and 20\% aqueous methanol [5]. Subaerial parts were extracted with methanol at room temperature [36].

Aerial part $n$-hexane extract is reported to contain lupeol, lupeol acetate, and taraxasteryl acetate [27]. In the aqueous ethanol extract, chlorogenic acid, hyperoside, and cyranoside were detected [5].

In the roots, the presence of taraxasteryl acetate, $3 \beta$-hydroxy-fern-7-en-6-one-acetate, olean-12-en-11-one-3-acetyl, lupeol, lupeol acetate, and $\alpha$-amyrin was detected in the n-hexane extract [27]. The aqueous methanol extract was also reported to contain chlorogenic acid [5], ( \pm -scorzotomentosin, (-)-,scorzotomentosin, (-)-scorzotomentosin 4'-O- $\beta$ glucoside, $( \pm)$-scorzophtalide, scorzoerzincanin, $( \pm)$-hydrangenol, (-)-hydrangenol 4 '-Oglucoside, $( \pm)$-hydramacrophyllol A, and $( \pm)$-hydramacrophyllol B [36].

\subsection{Scorzonera trachysperma Guss.}

Aerial parts of $S$. trachysperma samples, collected in Italy, were extracted with 50\% aqueous methanol and subjected to HPLC.

The analysis revealed that the aerial part methanol:water extract contains chlorogenic acid, cis-chlorogenic acid, cryptochlorogenic acid, isochlorogenic acid A, isochlorogenic acid C, 3,5-dicaffeoylquinic acid, isoorientin, luteolin, and apigenin diglycosides, and luteolin [26].

\subsection{Scorzonera undulata ssp. alexandrina Boiss.}

Samples of the plant were collected in Algeria and the whole plant was macerated with petroleum ether.

Lupeol, 24-methylenecycloartanol, 3-O-(6-O-acetyl- $\beta$-D-glucopyranosyl)- $\beta$-sitosterol, daucosterol, and apigenin were isolated from $S$. undulata ssp. alexandrina whole plant petroleum ether extract [23].

\subsection{Scorzonera undulata ssp. deliciosa (Guss.) Marie}

Plant samples were collected in Algeria and subaerial parts were macerated in dichloromethane, then the isolation of compounds was performed.

From the roots of the plant, the following compounds were isolated: verbascoside, galangustin [49], cichoriin, $\beta$-amyrin acetate, $\beta$-sitosterol, stigmasterol methyl oleanate, ethyl ursolate [63].

\subsection{Scorzonera veratrifolia Fenzl.}

The samples used for the studies on S. veratrifolia were collected in eastern Turkey. The subaerial parts were separated from the aerial parts and extracted with methanol at room temperature [30,64].

Subaerial parts of $S$. veratrifolia contain the following triterpenes: $\alpha$-amyrin acetate, $\alpha$-amyrinone, $\beta$-amyrin acetate, $\beta$-amyrinone, $\beta$-amyrin, $\psi$-taraxasterol, $\psi$-taraxasterol acetate, fern-7-en-3-one, germanicol, germanicol acetate, germanicone, lupenone, lupeol, lu- 
peol acetate, taraxasterol, and taraxasterol acetate, as well as $\beta$-sitosterol [30]. The presence of chlorogenic acid, chlorogenic acid methyl ester, isochlorogenic acid A, cryptochlorogenic acid, 4,5-dicaffeoylquinic acid, together with scorzoveratrin and scorzoveratrozit has also been reported [64].

\subsection{Scorzonera villosa Scop. ssp. villosa}

Samples of S. villosa were collected in Slovenia. Aerial parts were extracted with 50\% aqueous methanol at room temperature and HPLC analysis was performed.

The extract of aerial parts was reported to contain 1,5-O-dicaffeoylquinic acid, 3,5-Odicafeoylquinic acid, 5-O-caffeoylquinic acid, chlorogenic acid, cryptochlorogenic acid, apigenin 7-O-glucuronide, apigenin di-C-glycoside, apigetrin, cyranoside, hyperoside, isochlorogenic acid $\mathrm{C}$, isoorientin, isoquercitrin, luteolin, luteolin 7-O-glucuronide, and vitexin [26].

Compounds present in aerial parts of species within the genus Scorzonera, together with the concentration in the dry plant matter and the solvent used in the process of extraction (if available in the literature) are listed in Table 1. The phytochemical composition of subaerial parts of species belonging to the genus, with concentrations and solvents used, is presented in Table 2. Compounds isolated from the whole plants are presented in Table 3. As was presented in Tables 1 and 2, subaerial parts of the species within the genus Scorzonera are reported to contain a greater diversity of triterpenoid ad phenolic acid derivatives. This could be explained by the fact that a larger range of solvents was used for the extraction of phytochemicals from subaerial parts. The aerial parts; however, are reported to contain notably more different flavonoids. This is an anticipated outcome because, as flavonoid compounds are involved in biochemical processes within the whole plant, they are significant for the activities related to exposure to external factors (e.g., UV radiation or attracting pollinators) $[65,66]$. Another reason is the fact that only aerial parts of Scorzonera species were thoroughly assessed for flavonoid content in a study by Granica et al. [26]. Based on the research included in this review, the steroid, coumarin, and dihydroisocoumarin content seems similar in both aerial and subaerial parts. The species that are most investigated in the greatest number of papers are $S$. divaricata $[14,34,57-59]$, S. hispanica $[20,26,32,45,46,60]$, S. latifolia $[3-6,27,47,67-71]$, and S. radiata $[12,13,15]$. 
Table 1. Compounds present in aerial parts of Scorzonera species.

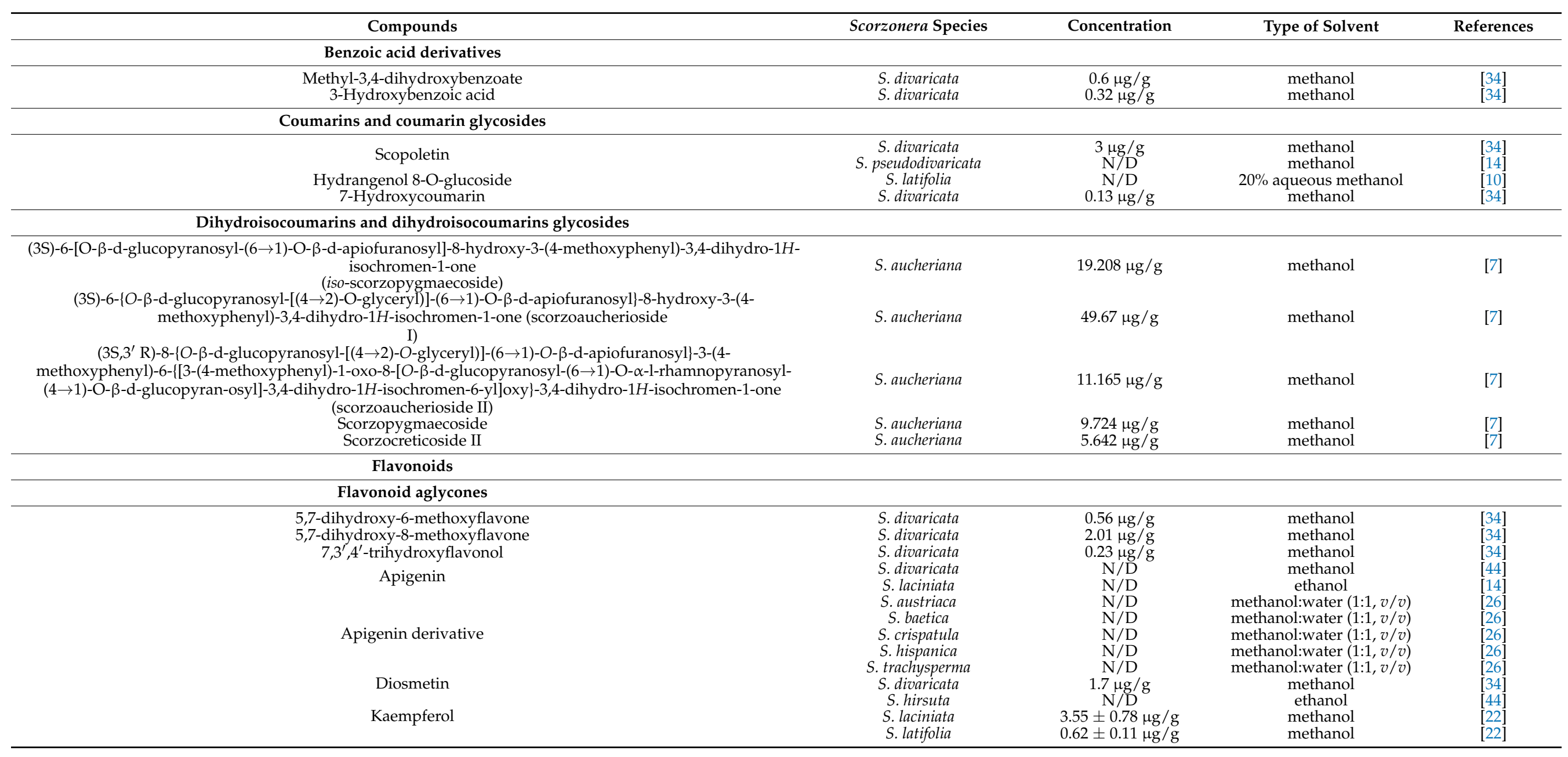


Table 1. Cont.

\begin{tabular}{|c|c|c|c|c|}
\hline Compounds & Scorzonera Species & Concentration & Type of Solvent & References \\
\hline $\begin{array}{l}\text { Luteolin } \\
\text { 2-(2,4-dihydroxyphenyl)-3,5,7-trihydroxychromen-4-one (morin) } \\
\text { Myricetin } \\
\text { Quercetin } \\
\text { Quercetin derivative } \\
\text { Tricin } \\
\text { Unknown flavonoid }\end{array}$ & $\begin{array}{c}\text { S. crispatula } \\
\text { S. divaricata } \\
\text { S. graminifolia } \\
\text { S. hirsuta } \\
\text { S. laciniata } \\
\text { S. mollis } \\
\text { S. pseudodivaricata } \\
\text { S. pussila } \\
\text { S. trachysperma } \\
\text { S. villosa } \\
\text { S. laciniata } \\
\text { S. latifolia } \\
\text { S. suberosa } \\
\text { S. laciniata } \\
\text { S. latifolia } \\
\text { S. suberosa } \\
\text { S. austriaca var. angustifolia } \\
\text { S. crispatula } \\
\text { S. graminifolia } \\
\text { S. hirsuta } \\
\text { S. hispanica } \\
\text { S. laciniata } \\
\text { S. latifolia } \\
\text { S. mollis } \\
\text { S. pussila } \\
\text { S. suberosa } \\
\text { S. aristata } \\
\text { S. austriaca } \\
\text { S. divaricata } \\
\text { S. hispanica } \\
\text { S. trachysperma } \\
\text { S. villosa }\end{array}$ & $\begin{array}{c}\text { N/D } \\
0.21 \mu g / g \\
\text { N/D } \\
\text { N/D } \\
\text { N/D } \\
\text { N/D } \\
\text { N/D } \\
\text { N/D } \\
\text { N/D } \\
\text { N/D } \\
0.17 \pm 0.01 \mu \mathrm{g} / \mathrm{g} \\
0.23 \pm 0.04 \mu \mathrm{g} / \mathrm{g} \\
0.91 \pm 0.83 \mu \mathrm{g} / \mathrm{g} \\
4.45 \pm 0.9 \mu \mathrm{g} / \mathrm{g} \\
16.16 \pm 0.92 \mu \mathrm{g} / \mathrm{g} \\
3.12 \pm 1.02 \mu \mathrm{g} / \mathrm{g} \\
\mathrm{N} / \mathrm{D} \\
\mathrm{N} / \mathrm{D} \\
\mathrm{N} / \mathrm{D} \\
\mathrm{N} / \mathrm{D} \\
\mathrm{N} / \mathrm{D} \\
0.17 \pm 0.01 \mu \mathrm{g} / \mathrm{g} \\
0.65 \pm 0.15 \mu \mathrm{g} / \mathrm{g} \\
\mathrm{N} / \mathrm{D} \\
\mathrm{N} / \mathrm{D} \\
6.54 \pm 16 \mu \mathrm{g} / \mathrm{g} \\
\mathrm{N} / \mathrm{D} \\
\mathrm{N} / \mathrm{D} \\
0.16 \mu \mathrm{g} / \mathrm{g} \\
\text { N/D } \\
\text { N/D } \\
\text { N/D }\end{array}$ & $\begin{array}{c}\text { methanol:water }(1: 1, v / v) \\
\text { methanol } \\
\text { ethanol } \\
\text { ethanol } \\
\text { ethanol } \\
\text { ethanol } \\
\text { methanol } \\
\text { ethanol } \\
\text { methanol:water }(1: 1, v / v) \\
\text { methanol:water }(1: 1, v / v) \\
\text { methanol } \\
\text { methanol } \\
\text { methanol } \\
\text { methanol } \\
\text { methanol } \\
\text { methanol } \\
\text { ethanol } \\
\text { methanol:water }(1: 1, v / v) \\
\text { ethanol } \\
\text { ethanol } \\
\text { methanol:water }(1: 1, v / v) \\
\text { methanol } \\
\text { methanol } \\
\text { ethanol } \\
\text { ethanol } \\
\text { methanol } \\
\text { methanol:water }(1: 1, v / v) \\
\text { methanol:water }(1: 1, v / v) \\
\text { methanol } \\
\text { methanol:water }(1: 1, v / v) \\
\text { methanol:water }(1: 1, v / v) \\
\text { methanol:water }(1: 1, v / v)\end{array}$ & $\begin{array}{l}{[26]} \\
{[34]} \\
{[44]} \\
{[44]} \\
{[44]} \\
{[44]} \\
{[14]} \\
{[44]} \\
{[26]} \\
{[26]} \\
{[22]} \\
{[22]} \\
{[22]} \\
{[22]} \\
{[22]} \\
{[22]} \\
{[44]} \\
{[26]} \\
{[44]} \\
{[44]} \\
{[26]} \\
{[22]} \\
{[22]} \\
{[44]} \\
{[44]} \\
{[22]} \\
{[26]} \\
{[26]} \\
{[34]} \\
{[26]} \\
{[26]} \\
{[26]}\end{array}$ \\
\hline \multicolumn{5}{|l|}{ Flavonoid $C$-glycosides } \\
\hline  & $\begin{array}{l}\text { S. austriaca } \\
\text { S. austriaca } \\
\text { S. austriaca } \\
\text { S. austriaca } \\
\text { S. austriaca } \\
\text { S. austriaca } \\
\text { S. radiata } \\
\text { S. latifolia } \\
\text { S. latifolia } \\
\text { S. tomentosa } \\
\text { S. radiata }\end{array}$ & $\begin{array}{l}10 \mu \mathrm{g} / \mathrm{g} \\
100 \mu \mathrm{g} / \mathrm{g} \\
15 \mu \mathrm{g} / \mathrm{g} \\
15 \mu \mathrm{g} / \mathrm{g} \\
15 \mu \mathrm{g} / \mathrm{g} \\
30 \mu \mathrm{g} / \mathrm{g} \\
\mathrm{N} / \mathrm{D} \\
\\
6.4 \mu \mathrm{g} / \mathrm{g} \\
\mathrm{N} / \mathrm{D} \\
\mathrm{N} / \mathrm{D} \\
7.334 \mu \mathrm{g} / \mathrm{g}\end{array}$ & $\begin{array}{c}70 \% \text { aqueous ethanol } \\
70 \% \text { aqueous ethanol } \\
70 \% \text { aqueous ethanol } \\
70 \% \text { aqueous ethanol } \\
70 \% \text { aqueous ethanol } \\
70 \% \text { aqueous ethanol } \\
\text { methanol } \\
\text { methanol } \\
20 \% \text { aqueous methanol } \\
20 \% \text { aqueous methanol } \\
\text { methanol }\end{array}$ & $\begin{array}{l}{[24]} \\
{[24]} \\
{[24]} \\
{[24]} \\
{[24]} \\
{[24]} \\
{[13]} \\
{[4]} \\
{[10]} \\
{[10]} \\
{[13]}\end{array}$ \\
\hline
\end{tabular}


Table 1. Cont.

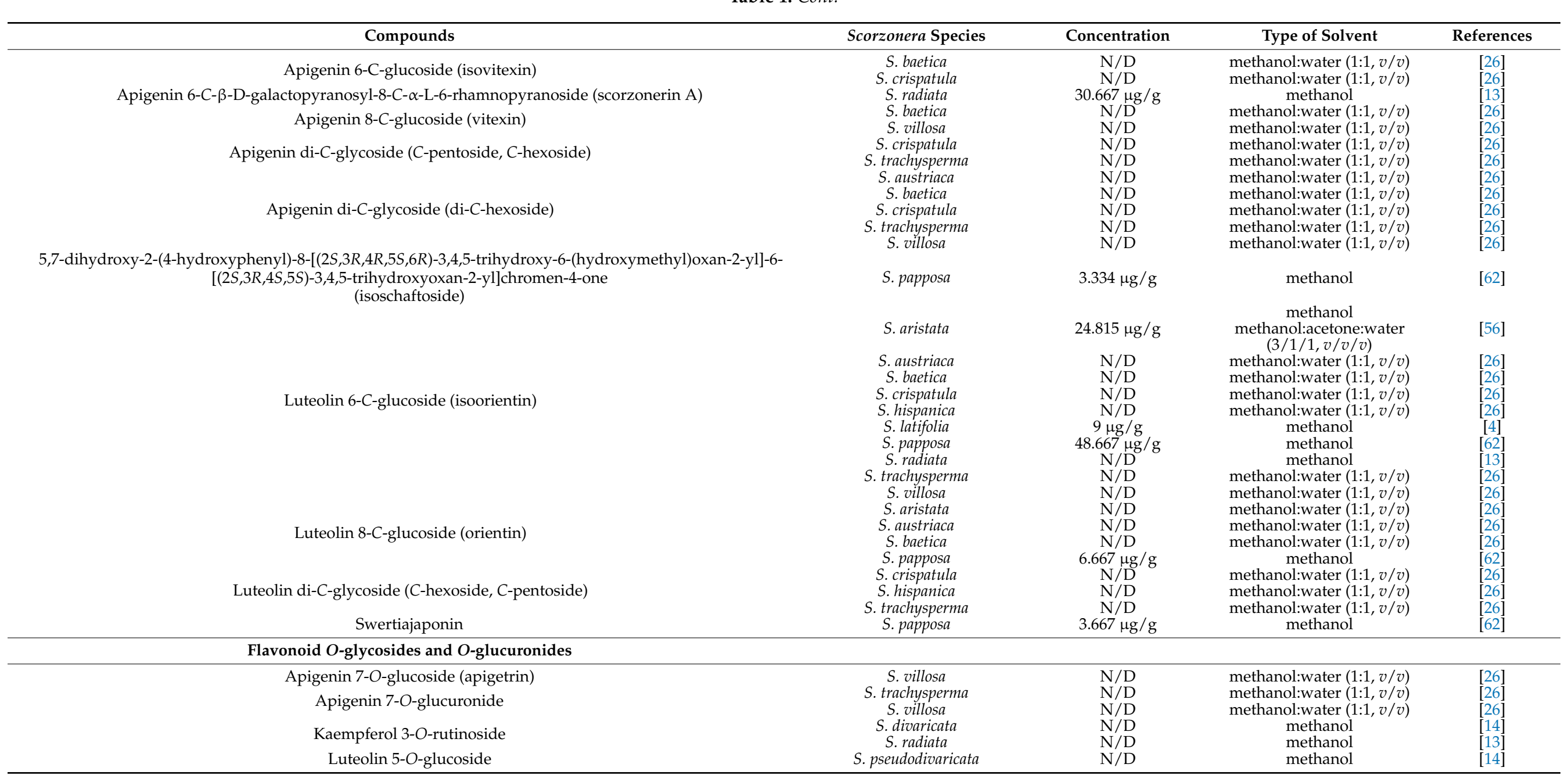


Table 1. Cont.

\section{Compounds}

Luteolin 7-O-glucoside (cynaroside)

Luteolin 7-O-glucuronide

Quercetin 3-O-arabinofuranoside (avicularin)

Quercetin 3-O-galactoside (hyperoside)

Quercetin 3-O-glucoside (isoquercitrin)

Quercetin 3-O-glucuronide (miquelianin)

Quercetin 3-O-rhamnoglucoside (rutin)

Quercetin 3-O- $\alpha$-rhamnopyranosyl- $(1 \rightarrow 6)-\beta$-D-galactopyranoside Quercetin 3-O- $\beta$-apiofuranosyl- $\left(1^{\prime \prime \prime} \rightarrow 2^{\prime \prime}\right)-\beta$-D-glucopyranoside

Quercetin 3-O- $\beta$-D-glucoside

Quercetin O-mallonylhexoside

\begin{tabular}{|c|c|c|c|}
\hline Scorzonera Species & Concentration & Type of Solvent & References \\
\hline S. acuminata & $9.583 \pm 0.203 \mu \mathrm{g} / \mathrm{mg}$ & $20 \%$ aqueous methanol & [9] \\
\hline S. baetica, & N/D & methanol:water $(1: 1, v / v)$ & [26] \\
\hline S. cinerea & $81.14 \pm 0.62 \mu \mathrm{g} / \mathrm{mg}$ & $20 \%$ aqueous methanol & [5] \\
\hline S. incisa & $12.08 \pm 0.1 \mu \mathrm{g} / \mathrm{mg}$ & $20 \%$ aqueous methanol & [5] \\
\hline S. latifolia & $629.23 \pm 3.53 \mu \mathrm{g} / \mathrm{mg}$ & $20 \%$ aqueous methanol & [5] \\
\hline S. mollis ssp. szowitsii & $107.43 \pm 0.09 \mu \mathrm{g} / \mathrm{mg}$ & $20 \%$ aqueous methanol & [5] \\
\hline $\begin{array}{l}\text { S. parviflora } \\
\text { Sseudodizoricata }\end{array}$ & $51.80 \pm 0.71 \mu \mathrm{g} / \mathrm{mg}$ & $20 \%$ aqueous methanol & [5] \\
\hline S. pseudodivaricata & $\mathrm{N} / \mathrm{D}$ & methanol & [14] \\
\hline $\begin{array}{l}\text { S. tomentosa } \\
\text { S. villosa }\end{array}$ & $47.81 \pm 0.50 \mu \mathrm{g} / \mathrm{mg}$ & $20 \%$ aqueous methanol & [5] \\
\hline S. trachysperma & $\begin{array}{l}\text { N/D } \\
\text { N/D }\end{array}$ & $\begin{array}{l}\text { methanol:water }(1: 1, v / v) \\
\text { methanol:water }(1: 1, v / v)\end{array}$ & \\
\hline S. villosa & N/D & $\begin{array}{l}\text { methanol:water }(1: 1, v / v) \\
\text { methanol:water }(1: 1, v / v)\end{array}$ & [26] \\
\hline S. austriaca & N/D & methanol:water $(1: 1, v / v)$ & [26] \\
\hline S. cinerea & $124.22 \pm 0.56 \mu \mathrm{g} / \mathrm{mg}$ & $20 \%$ aqueous methanol & [5] \\
\hline S. hispanica & $\begin{array}{l}11.41 \pm 0.05 \mathrm{mg} / \mathrm{g} \\
11.35 \pm 0.15 \mathrm{mg} / \mathrm{g}\end{array}$ & $\begin{array}{c}\text { methanol:acetone:water } \\
(3: 1 \cdot 1)\end{array}$ & [20] \\
\hline S. latifolia & $305.71 \pm 1.70 \mu \mathrm{g} / \mathrm{mg}$ & $20 \%$ aqueous methanol & [5] \\
\hline S. mollis ssp. szowitsii & $39.46 \pm 0.03 \mu \mathrm{g} / \mathrm{mg}$ & $20 \%$ aqueous methanol & [5] \\
\hline S. parviflora & $9.71 \pm 0.51 \mu \mathrm{g} / \mathrm{mg}$ & $20 \%$ aqueous methanol & [5] \\
\hline $\begin{array}{l}\text { S. tomentosa } \\
\text { S. villosa }\end{array}$ & $\begin{array}{c}94.54 \pm 0.33 \mu \mathrm{\mu} / \mathrm{mg} \\
\mathrm{N} / \mathrm{D}\end{array}$ & $\begin{array}{l}20 \% \text { aqueous methanol } \\
\text { methanol:water }(1: 1, v / v)\end{array}$ & {$\left[\begin{array}{l}{[5]} \\
{[26]}\end{array}\right.$} \\
\hline S. aristata & $40.37 \mu \mathrm{g} / \mathrm{g}$ & $\begin{array}{c}\text { methanol } \\
\text { methanol:acetone:water } \\
(3 / 1 / 1, v / v / v)\end{array}$ & [56] \\
\hline S. austriaca & N/D & methanol:water $(1: 1, v / v)$ & [26] \\
\hline S. hispanica & $\begin{array}{l}6.41 \pm 0.02 \mathrm{mg} / \mathrm{g} \\
6.91 \pm 0.01 \mathrm{mg} / \mathrm{g}\end{array}$ & $\begin{array}{c}\text { methanol:acetone:water } \\
(3 / 1 / 1, v / v / v)\end{array}$ & [20] \\
\hline S. villosa & N/D & methanol:water $(1: 1, v / v)$ & [26] \\
\hline S. hispanica & N/D & methanol:water $(1: 1, v / v)$ & [26] \\
\hline & $\begin{array}{l}13.87 \pm 0.10 \mathrm{mg} / \mathrm{g} \\
15.29 \pm 0.25 \mathrm{mg} / \mathrm{g}\end{array}$ & $\begin{array}{c}\text { methanol:acetone:water } \\
(3 / 1 / 1, v / v / v)\end{array}$ & [20] \\
\hline S. acuminata & $597.335 \pm 1.104 \mu \mathrm{g} / \mathrm{mg}$ & $\begin{array}{l}20 \% \text { aqueous methanol } \\
\text { methanol }\end{array}$ & [9] \\
\hline S. aristata & $36.667 \mu \mathrm{g} / \mathrm{g}$ & $\begin{array}{c}\text { methanol:acetone:water } \\
(3 / 1 / 1, v / v / v)\end{array}$ & [56] \\
\hline S. austriaca & $\mathrm{N} / \mathrm{D}$ & methanol:water $(1: 1, v / v)$ & [26] \\
\hline & $198.81 \pm 0.18 \mu \mathrm{g} / \mathrm{mg}$ & $20 \%$ aqueous methanol & [5] \\
\hline S. mollis ssp. szowitsii & $26.32 \pm 0.04 \mu \mathrm{g} / \mathrm{mg}$ & $20 \%$ aqueous methanol & [5] \\
\hline S. radiata & $\mathrm{N} / \mathrm{D}$ & methanol & [13] \\
\hline S. suberosa & $15.38 \pm 3.27 \mu \mathrm{g} / \mathrm{g}$ & methanol & [22] \\
\hline S. latifolia & $2.267 \mu \mathrm{g} / \mathrm{g}$ & methanol & [4] \\
\hline S. latifolia & $11.53 \mu \mathrm{g} / \mathrm{g}$ & methanol & [4] \\
\hline S. latifolia & $\mathrm{N} / \mathrm{D}$ & $20 \%$ aqueous methanol & ]10] \\
\hline S. tomentosa & $\mathrm{N} / \mathrm{D}$ & $20 \%$ aqueous methanol & {$[10]$} \\
\hline S. aristata & $\mathrm{N} / \mathrm{D}$ & methanol:water $(1: 1, v / v)$ & [26] \\
\hline S. hispanica & $\begin{array}{l}2.65 \pm 0.05 \mu \mathrm{g} / \mathrm{mg} \\
2.83 \pm 0.01 \mu \mathrm{g} / \mathrm{mg}\end{array}$ & $\begin{array}{c}\text { methanol:acetone:water } \\
(3 / 1 / 1, v / v / v)\end{array}$ & [20] \\
\hline
\end{tabular}


Table 1. Cont.

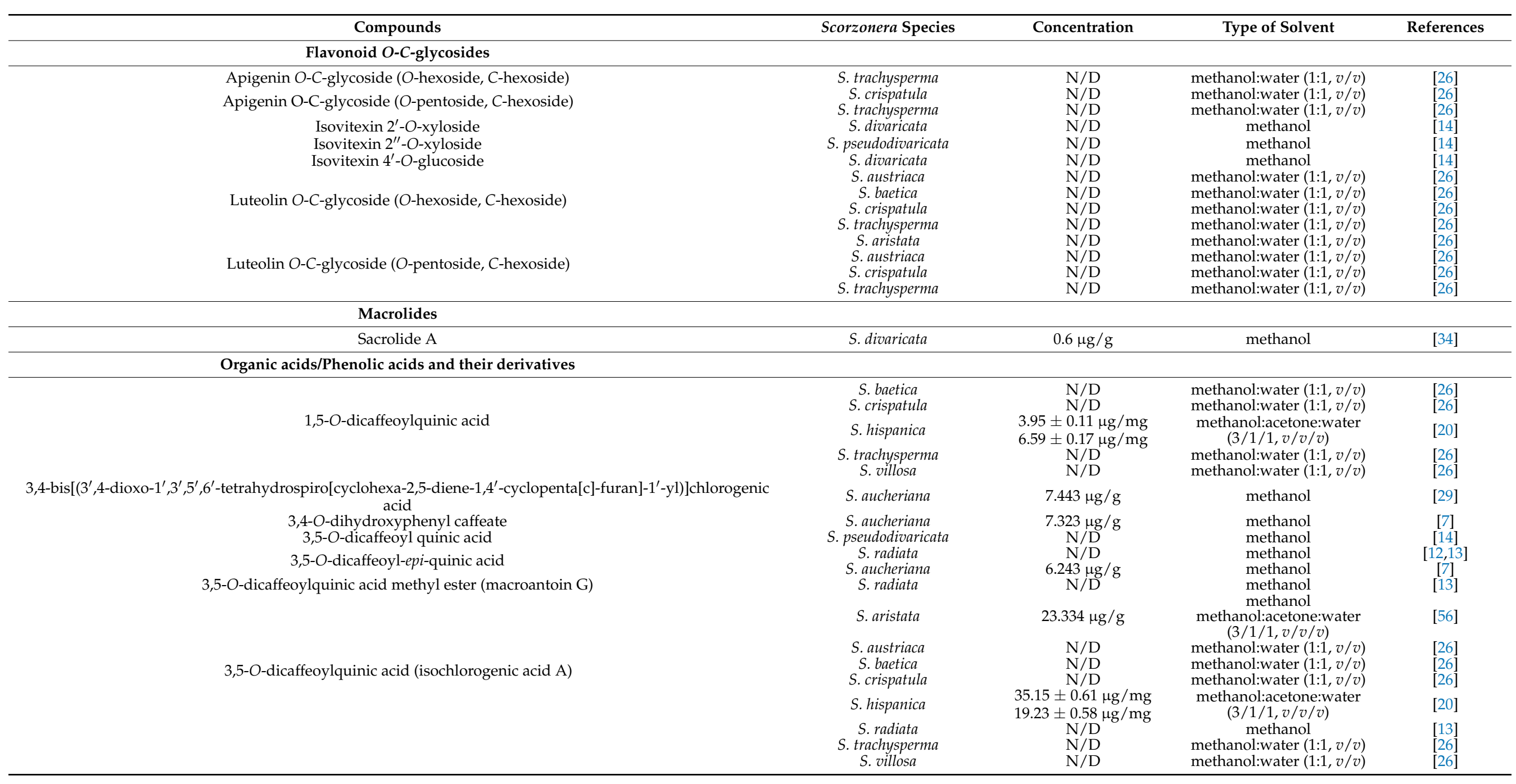


Table 1. Cont.

\section{Compounds}

3-O-caffeoylquinic acid (chlorogenic acid)

3-O-feruloyl-1,4-di-O-dihydrocaffeoylquinic acid (feruloylpodospermic acid B) 3-O-feruloyl-1,5-di-O-dihydrocaffeoylquinic acid (feruloylpodospermic acid A) ,5-dicaffeoyl-epi-quinic acid

4,5-dicaffeoyl-epi-quinic acid methyl ester (macroantoin F)

4,5-O-dicaffeoylquinic acid (isochlorogenic acid C)

4-hydroxy-3-methoxyphenyl ferulate

4-O-caffeoylquinic acid (cryptochlorogenic acid)

5-O-caffeoylquinic acid (cis-chlorogenic acid)

Scorzonera Species
S. acuminata
S. aristata
S. austriaca
S. baetica
S. cinerea
S. crispatula
S. divaricata
S. hispanica
S. incisa
S. latifolia
S. mollis ssp. szowitsii
S. parviflora
S. radiata
S. tomentosa
S. trachysperma
S. villosa
S. divaricata
S. divaricata
S. radiata
S. radiata
S. aristata
S. austriaca
S. baetica
S. crispatula
S. hispanica
S. radiata
S. trachysperma
S. villosa
S. divaricata
S. aristata
S. austriaca
S. baetica
S. crispatula
S. hispanica
S. trachysperma
S. villosa
S. baetica
S. crispatula
S. hispanica
S. trachysperma
S. villosa
ald

Concentration

$372.128 \pm 0.961 \mu \mathrm{g} / \mathrm{mg}$

$$
9.259 \mu \mathrm{g} / \mathrm{g}
$$$$
\mathrm{N} / \mathrm{D}
$$$$
266.51 \pm 1.51 \mu \mathrm{g} / \mathrm{mg}
$$$$
\mathrm{N} / \mathrm{D}
$$$$
85.49 \pm 1.49 \mu \mathrm{g} / \mathrm{mg}
$$

$85.49 \pm 1.49 \mu \mathrm{g} / \mathrm{mg}$
$75.83 \pm 1.01 \mu \mathrm{g} / \mathrm{mg}$

$569.19 \pm 1.62 \mu \mathrm{g} / \mathrm{mg}$

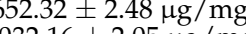

$444.77 \pm 2.78 \mu \mathrm{g} / \mathrm{mg}$$$
\text { N/D }
$$

$268.75 \pm 1.72 \mu \mathrm{g} / \mathrm{mg}$

N/D

$23.438 \mathrm{\mu g} / \mathrm{g}$

$82.031 \mu \mathrm{g} / \mathrm{g}$

$10.333 \mu \mathrm{g} / \mathrm{g}$
$\mathrm{N} / \mathrm{D}$

$13.33 \mu \mathrm{g} / \mathrm{g}$

$\mathrm{N} / \mathrm{D}$

N/D

$5.42 \pm 0.01 \mu \mathrm{g} / \mathrm{mg}$

$3.14 \pm 0.15 \mu \mathrm{g} / \mathrm{mg}$

N/D

N/D

$0.13 \mu \mathrm{g} / \mathrm{g}$

N/D

$\mathrm{N} / \mathrm{D}$
$\mathrm{N} / \mathrm{D}$
$\mathrm{N} / \mathrm{D}$

$2.99 \pm 0.03 \mu \mathrm{g} / \mathrm{mg}$

$3.99 \pm 0.04 \mu \mathrm{g} / \mathrm{mg}$

N/D

N/D

N/D

N/D

N/D
Type of Solvent

$20 \%$ aqueous methanol methanol

methanol:acetone:water

$(3 / 1 / 1, v / v / v)$

methanol:water $(1: 1, v / v)$

methanol:water $(1: 1, v / v)$

$20 \%$ aqueous methanol nol:water $(1: 1, v / v)$ methanol

methanol:acetone:water

$(3 / 1 / 1, v / v / v)$

$20 \%$ aqueous methanol

$20 \%$ aqueous methanol

$20 \%$ aqueous methanol

$$
\text { methanol }
$$

$20 \%$ aqueous methanol

methanol:water $(1: 1, v / v)$ methanol:water $(1: 1, v / v)$

$$
\text { methanol }
$$

methanol

methanol

$$
\text { methanol }
$$

methanol:acetone:water

$(3 / 1 / 1, v / v / v)$

methanol:water $(1: 1, v / v)$

methanol:water $(1: 1, v / v)$

methan.water $(1: 1,0 / 0)$

thanol:acetone:wa (3/1, $0 / 0 / \%)$

methanol:water $(1: 1, v / v)$

methanol:water $(1: 1, v / v)$
methanol:water $(1: 1, v / v)$

methanol

methanol:water $(1: 1, v / v)$

methanol:water $(1: 1, v / v)$

methanol:water $(1: 1, v / v)$

methanol:water $(1: 1, v / v)$

methanol:acetone:wate

ethanol:water $(1: 1, v / v)$

methanol:water $(1: 1, v / v)$

methanol:water $(1: 1, v / v)$

methanol:water $(1: 1, v / v)$

methanol:water $(1: 1, v / v)$

methanol:water $(1: 1, v / v)$
methanol:water $(1: 1, v / v)$
References

[9]

[56]

[26]

[5]

[20]

[5]

$[5]$
$[5]$
$[5]$
55 
Table 1. Cont.

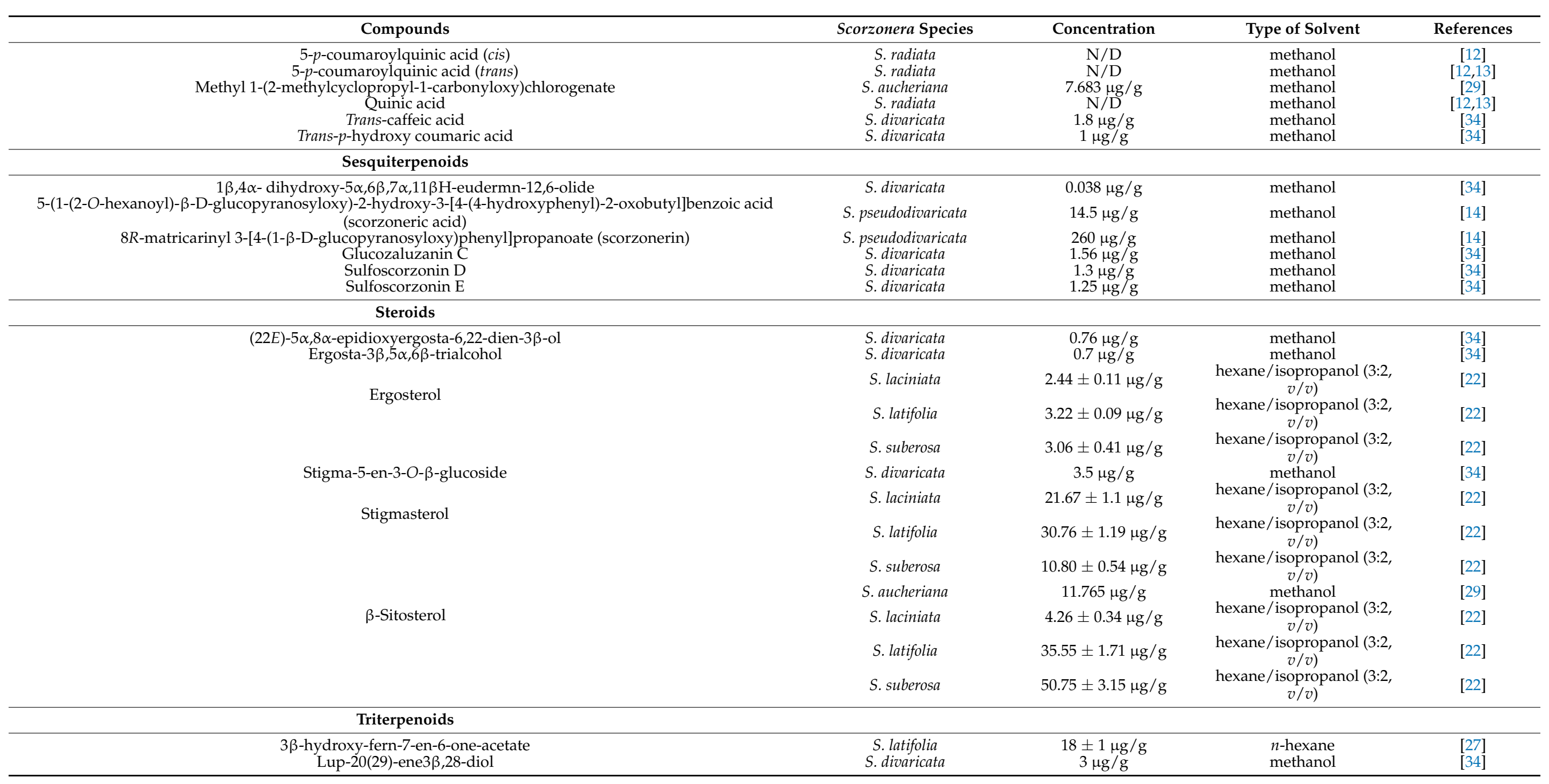


Table 1. Cont

Compounds

Lupeol

Lupeol acetate

Oleanolic acid Ptiloepoxide
Taraxasterol

Taraxasteryl acetate/Taraxasterol acetate

Taraxasterol oleat
Scorzonera Species

S. acuminata
S. aucherian

S. cana var. jacquiniana S. cinerea
S. eriophora

S. eriophora
S. incisa

S. laciniata ssp. laciniata S. latifolia

S. mirabilis
Sollis ssp. szowitsii

S. parviflora
S. suberosa ssp. suberosa

S. sublanata

S. tomentosa

S. acuminata

S. cana var. jacquiniana S. cinerea S. eriophora
S. incisa

S. laciniata ssp. laciniata S. latifolia
S. mirabilis

S. mirabilis
Sollis ssp. szowitsii S. parviflora

S. suberosa ssp. suberosa S. sublanata

S. tomentosa

S. divaricata

S. aucheriana

S. aucheriana

S. cana var. jacquiniana S. cinerea S. eriophora
S. incisa

S. laciniata ssp. laciniata S. latifolia
S. mirabilis

S. mollis ssp. szowitsii S. parviflora

S. suberosa ssp. suberosa

S. sublanata

S. tomentosa
S. aucheriana
Concentration

$327 \pm 5 \mu \mathrm{g} / \mathrm{g}$
$9.724 \mu \mathrm{g} / \mathrm{g}$

$932 \pm 2 \mu \mathrm{g} / \mathrm{g}$

$1174 \pm 16 \mu \mathrm{g} / \mathrm{g}$

$228 \pm 6 \mu \mathrm{g} / \mathrm{g}$

$1090 \pm 2 \mu \mathrm{g} / \mathrm{g}$

$932 \pm 2 \mu \mathrm{g} / \mathrm{g}$
$1538 \pm 1 \mu \mathrm{g} / \mathrm{g}$

$954 \pm 14 \mu \mathrm{g} / \mathrm{g}$

$321 \pm 1 \mu \mathrm{g} / \mathrm{g}$

$649 \pm 6 \mu \mathrm{g} / \mathrm{g}$

$169+1 \mu \mathrm{g} / \mathrm{g}$

$509 \pm 2 \mu \mathrm{g} / \mathrm{g}$

$67 \pm 1 \mu \mathrm{g} / \mathrm{g}$

$5.642 \mu \mathrm{g} / \mathrm{g}$

$535 \pm 4 \mu \mathrm{g} / \mathrm{g}$
$839 \pm 6 \mu \mathrm{g} / \mathrm{g}$

$368 \pm 1 \mu \mathrm{g} / \mathrm{g}$

$236 \pm 9 \mu \mathrm{g} / \mathrm{g}$

$892 \pm 2 \mu \mathrm{g} / \mathrm{g}$

$607 \pm 1 \mu \mathrm{g} / \mathrm{g}$

$998 \pm 13 \mu \mathrm{g} / \mathrm{g}$

$149 \pm 7 \mu \mathrm{g} / \mathrm{g}$

$594 \pm 5 \mu \mathrm{g} / \mathrm{g}$

$312 \pm 4 \mu \mathrm{g} / \mathrm{g}$
$302 \pm 1 \mu \mathrm{g} / \mathrm{g}$

$302 \pm 1 \mu \mathrm{g} / \mathrm{g}$

$11 \pm 1 \mu \mathrm{\mu g} / \mathrm{g}$
$1.5 \mu \mathrm{g} / \mathrm{g}$

$14.646 \mu \mathrm{g} / \mathrm{g}$

$30.972 \mu \mathrm{g} / \mathrm{g}$

$81 \pm 3 \mu \mathrm{g} / \mathrm{g}$

$81 \pm 3 \mu \mathrm{g} / \mathrm{g}$
$417 \pm 11 \mu \mathrm{g} / \mathrm{g}$

$417 \pm 11 \mu \mathrm{g} / \mathrm{g}$

$545 \pm 5 \mu \mathrm{g} / \mathrm{g}$
$280 \pm 10 \mu \mathrm{g} / \mathrm{g}$

$69 \pm 5 \mu \mathrm{g} / \mathrm{g}$

$1062 \pm 2 \mu \mathrm{g} / \mathrm{g}$

$262 \pm 728 \mu \mathrm{g} / \mathrm{g}$

$263 \pm 4 \mu \mathrm{g} / \mathrm{g}$

$535 \pm 4 \mu \mathrm{g} / \mathrm{g}$

$4981 \pm 2 \mu \mathrm{g} / \mathrm{g}$

$376 \pm 13 \mu \mathrm{g} / \mathrm{g}$
$36.255 \mu \mathrm{g} / \mathrm{g}$
Type of Solvent

$n$-hexane
methanol

n-hexane

$n$-hexane

$n$-hexane

$n$-hexane

$n$-hexane

$n$-hexane

$n$-hexane

$n$-hexane

$n$-hexane

$n$-hexane

$n$-hexane

$n$-hexane

$n$-hexane
methanol

$n$-hexane

$n$-hexane

$n$-hexane

$n$-hexane

$n$-hexane

$n$-hexane

$n$-hexane

$n$-hexane

$n$-hexane

$n$-hexane

$n$-hexane

n-hexane

methanol

methanol

methanol

methanol

n-hexane

$n$-hexane

$n$-hexane

$n$-hexane

$n$-hexane

$n$-hexane

$n$-hexane

$n$-hexane

$n$-hexane

$n$-hexane

$n$-hexane
methanol
References

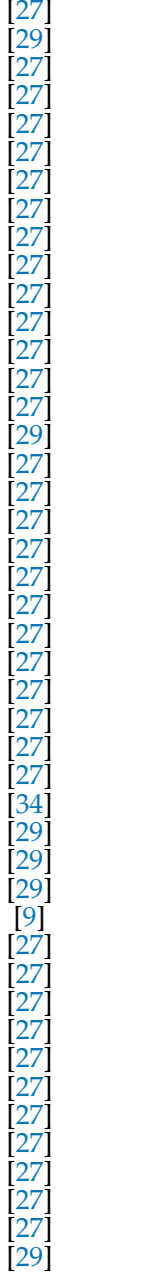


Table 1. Cont.

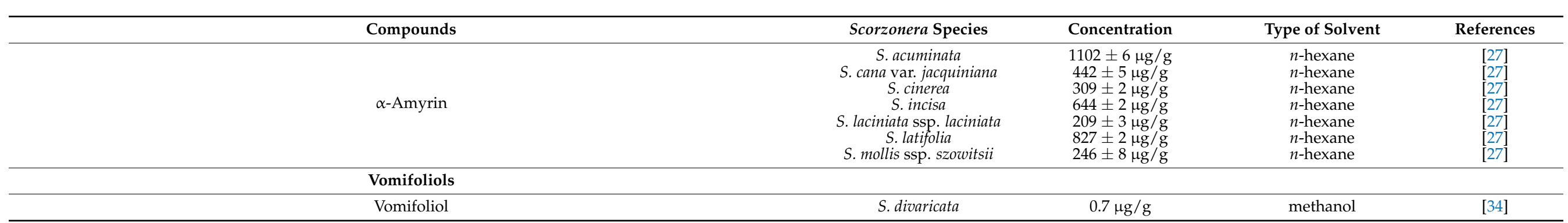

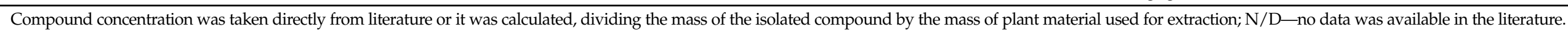

Table 2. Compounds present in subaerial parts of Scorzonera species.

\begin{tabular}{|c|c|c|c|c|}
\hline Compounds & Scorzonera Species & Concentration & Type of Solvent & References \\
\hline \multicolumn{5}{|l|}{ Coumarins and coumarin derivatives } \\
\hline Scopoletin & S. divaricata & $2.546 \mu \mathrm{g} / \mathrm{g}$ & $95 \%$ aqueous ethanol & [57] \\
\hline Scopolin & S. divaricata $\ldots$ & $16.364 \mu \mathrm{g} / \mathrm{g}$ & $95 \%$ aqueous ethanol & [57] \\
\hline Coumarin $O-\beta$-glycoside (cichoriin) & S. undulata ssp. deliciosa & $12.99 \mu \mathrm{g} / \mathrm{g}$ & dichloromethane & [63] \\
\hline Scorzonerol & $\begin{array}{l}\text { S. cana var. jacquiniana } \\
\text { S. pygmaea }\end{array}$ & $\begin{array}{l}6.667 \mu \mathrm{g} / \mathrm{g} \\
0.857 \mu \mathrm{g} / \mathrm{g}\end{array}$ & $\begin{array}{l}\text { methanol } \\
\text { ethanol }\end{array}$ & {$[32]$} \\
\hline \multicolumn{5}{|l|}{ Dihydroisocoumarins and dihydroisocoumarin glycosides } \\
\hline \multirow{2}{*}{ (-)-Hydrangenol 4'-O-glucoside } & S. tomentosa & $20.101 \mu \mathrm{g} / \mathrm{g}$ & methanol & [36] \\
\hline & S. judaica & $14.286 \mu \mathrm{g} / \mathrm{g}$ & $\begin{array}{l}\text { chloroform:methanol }(9: 1) \\
\text { methanol }\end{array}$ & [25] \\
\hline \multirow[t]{2}{*}{ (-)-Scorzotomentosin $4^{\prime}-O-\beta$-glucoside } & S. tomentosa & $158.29 \mu \mathrm{g} / \mathrm{g}$ & $\begin{array}{l}\text { methanol } \\
\text { methand.water }(8 \cdot 2)\end{array}$ & [36] \\
\hline & S. tomentosa & $82.915 \mu \mathrm{g} / \mathrm{g}$ & methanol & [36] \\
\hline$( \pm)$-Hydrangenol & S. judaica & $51.143 \mu \mathrm{g} / \mathrm{g}$ & $\begin{array}{l}\text { chloroform } \\
\text { chloroform:methanol (9:1) }\end{array}$ & [25] \\
\hline (3RS)-3,4-dihydro-3-(4-hydroxyphenyl)-8-methoxy-1H-2-benzopyran-1-one $(( \pm)$-scorzotomentosin) & $\begin{array}{l}\text { S. tomentosa } \\
\text { S. judaica }\end{array}$ & $\begin{array}{l}216.08 \mu \mathrm{g} / \mathrm{g} \\
8.714 \mu \mathrm{g} / \mathrm{g}\end{array}$ & $\begin{array}{l}\text { methanol } \\
\text { chloroform }\end{array}$ & {$[36]$} \\
\hline 8-O-[ $\alpha$-L-rhamnopyranosyl $(1 \rightarrow 6)-\beta$-D-glucopyranosyl] scorzocreticin (scorzocreticoside II) & S. pygmaea & $21.429 \mu \mathrm{\mu g} / \mathrm{g}$ & ethanol & {$[35]$} \\
\hline 8-O- $\beta$-D-glucopyranosylscorzocreticin (scorzocreticoside I) & S. pygmaea & $14.286 \mu \mathrm{g} / \mathrm{g}$ & $\begin{array}{l}\text { ethanol } \\
\text { chloroform:methanol }(9: 1)\end{array}$ & [35] \\
\hline Hydrangenol 4'-O- $\beta$-D-apiofuranosyl-( $1 \rightarrow 6)$ - $\beta$-D-glucopyranoside & S. judaica & $22.143 \mu \mathrm{g} / \mathrm{g}$ & $\begin{array}{l}\text { chloroform:methanol (9:1) } \\
\text { methanol }\end{array}$ & [25] \\
\hline 3S-hydrangenol 4'-O- $\alpha$-L-rhamnopyranosyl-(1 $\rightarrow 3)-\beta$-D-glucopyranoside & S. judaica & $48.571 \mu \mathrm{g} / \mathrm{g}$ & $\begin{array}{l}\text { chloroform:methanol (9:1) } \\
\text { methanol }\end{array}$ & [25] \\
\hline Scorzopygmaecoside & S. pygmaea & $14.286 \mu \mathrm{g} / \mathrm{g}$ & ethanol & [35] \\
\hline $\begin{array}{l}\text { Thunberginol C } \\
\text { Thunberginol F }\end{array}$ & $\begin{array}{l}\text { S. pygmaea } \\
\text { S. judaica }\end{array}$ & $4.714 \mu \mathrm{g} / \mathrm{g}$ & $\begin{array}{l}\text { ethanol } \\
\text { (a) }\end{array}$ & [35] \\
\hline $\begin{array}{l}\text { Thunberginol F } \\
\text { Thunberginol G }\end{array}$ & $\begin{array}{l}\text { S. judaica } \\
\text { S. papposa }\end{array}$ & $\begin{array}{l}7.429 \mu \mathrm{g} / \mathrm{g} \\
38.4 \mu \mathrm{g} / \mathrm{g}\end{array}$ & $\begin{array}{c}\text { chloroform:methanol (9:1) } \\
\text { methanol }\end{array}$ & {$[62]$} \\
\hline
\end{tabular}


Table 2. Cont

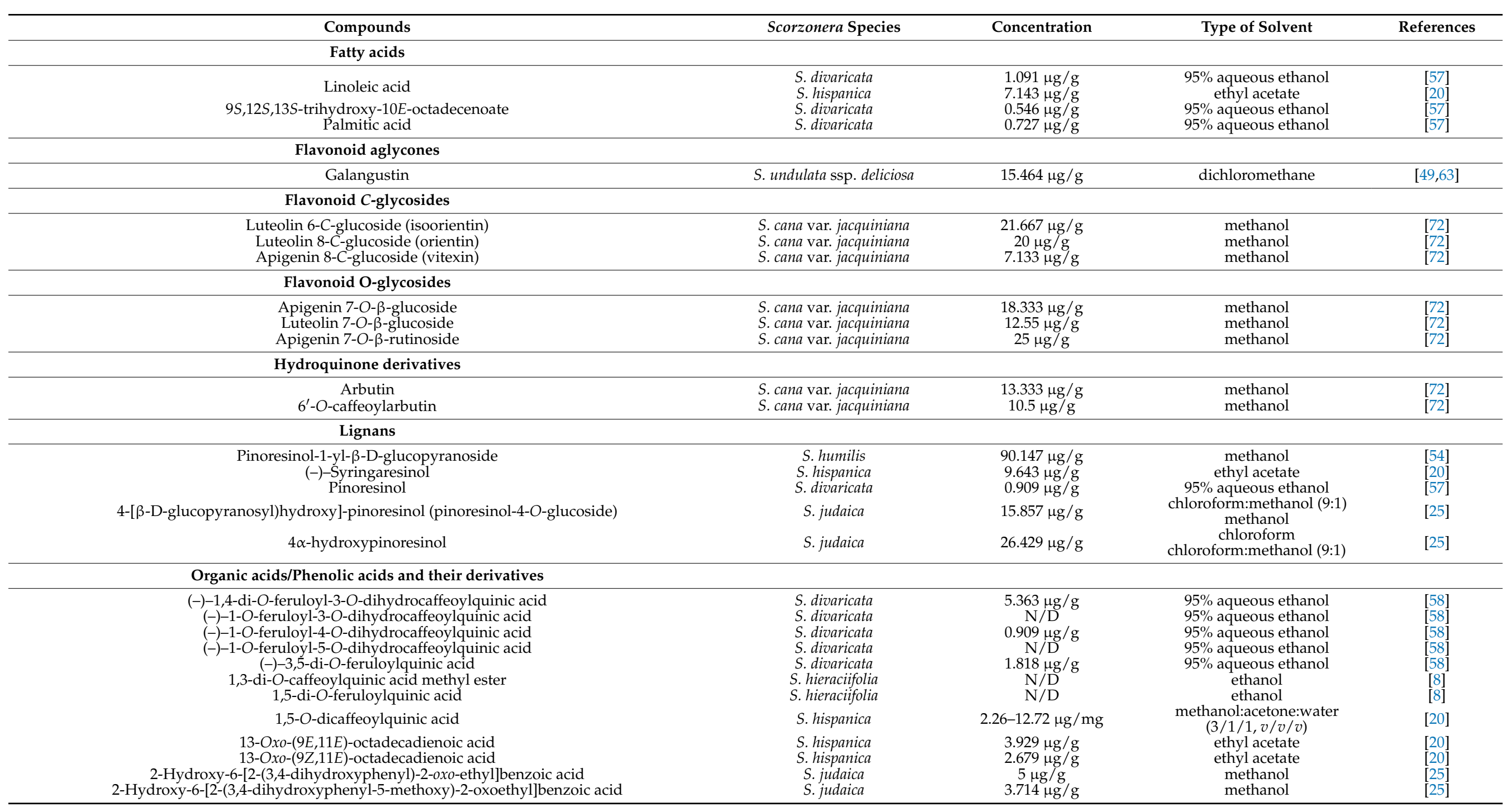


Table 2. Cont.

\section{Compounds}

2-Hydroxy-6-[2-(4-hydroxyphenyl)-2-oxo-ethyl]benzoic acid

3-(4'-Hydroxyphenyl)-2-propenoic acid (4'-carboxyl)-phenyl ester

3,5-di-O-caffeoylquinic acid

3,5-dicaffeoylquinic acid methyl ester (macroantoin G)

3-O-caffeoylquinic acid methyl ester

4,5-dicaffeoylquinic acid (isochlorogenic acid C)

4,5-di-O-caffeoylquinic acid methyl ester

4-Hydroxybenzoic acid 4-(6-O- $\alpha$-rhamnopyranosyl- $\beta$-glucopyranosyl) benzyl ester

4-O-caffeoylquinic acid (cryptochlorogenic acid)

5-O-feruloyl quinic acid methyl ester

9-Hydroxyocta-(10E,12E)-decadienoic acid

9-Oxo-(10E,12E)-octadecadienoic acid

Butyl 3-O-feruloylquinate

Caffeic acid

Caffeic acid methyl ester

Chlorogenic acid
Scorzon

Scorzonera Species

S. judaica
S. hieraciifolia
S. aristata
S. hispanica
S. humilis
S. latifolia
S. pygmaea
S. hier. jacquiiinoliana
S. hieraciifolia
S. hispanica
S. latifolia
S. veratrifolia
S. latifolia
S. hieraciifolia

S. cana var. jacquiniana

S. hispanica

S. hieraciifolia

S. hispanica

S. hispanica

S. hispanica

S. divaricata

S. hieraciifolia

S. hispanica

S. latifolia

S. aristata

S. cinerea

S. hispanica

S. humilis

S. incisa
S. latifolia

S. mollis ssp. szowitsii

S. parviflora

S. pygmaea

S. vomentosa

Concentration

$17.143 \mu \mathrm{g} / \mathrm{g}$

$\mathrm{N} / \mathrm{D}$

$13.735 \mu \mathrm{g} / \mathrm{g}$

$1.04-52.13 \mu \mathrm{g} / \mathrm{mg}$

$\mathrm{N} / \mathrm{D}$

$12.5 \mu \mathrm{g} / \mathrm{g}$
$3.214 \mu \mathrm{g} / \mathrm{g}$

$10 \mu \mathrm{g} / \mathrm{g}$

$\mathrm{N} / \mathrm{D}$

$2.46-4.59 \mu \mathrm{g} / \mathrm{mg}$ $2.5 \mu \mathrm{g} / \mathrm{g}$

$25.667 \mu \mathrm{g} / \mathrm{g}$ $25 \mu \mathrm{g} / \mathrm{g}$

$20.433 \mu \mathrm{g} / \mathrm{g}$

$0.52-0.93 \mu \mathrm{g} / \mathrm{mg}$

$\mathrm{N} / \mathrm{D}$
$\mathrm{N} / \mathrm{D}$
$1.071 \mu \mathrm{g} / \mathrm{g}$
$\mathrm{N} / \mathrm{D}$
$2.909 \mu \mathrm{g} / \mathrm{g}$

$2.909 \mu \mathrm{g} / \mathrm{g}$
$2.909 \mu \mathrm{g} / \mathrm{g}$

$\mathrm{N} / \mathrm{D}$

$0.13-2.47 \mu \mathrm{g} / \mathrm{g}$

$4.58 \mu \mathrm{g} / \mathrm{g}$

$56.627 \mu \mathrm{g} / \mathrm{g}$

$412.89 \pm 0.55 \mu \mathrm{g} / \mathrm{mg}$

$3.80-43.82 \mu \mathrm{g} / \mathrm{mg}$ N.D

$141.49 \pm 0.20 \mu \mathrm{g} / \mathrm{mg}$

$1246.78 \pm 3.20 \mu \mathrm{g} / \mathrm{mg}$

$159.25 \pm 0.24 \mu \mathrm{g} / \mathrm{mg}$

$509.96 \pm 6.64 \mu \mathrm{g} / \mathrm{mg}$

$3.43 \mu \mathrm{g} / \mathrm{g}$
$734.72 \pm 1.04 \mu \mathrm{g} / \mathrm{mg}$

\begin{tabular}{l}
$61.857 \mu \mathrm{g} / \mathrm{g}$ \\
\hline
\end{tabular}
Type of Solvent

References

chloroform:methanol (9:1)

$$
\begin{gathered}
\text { methanol } \\
\text { ethanol }
\end{gathered}
$$

methanol:acetone:water

$(3 / 1 / 1, v / v / v)$
methanol

ethanol:acetone:wate

$(3 / 1 / 1, v / v / v)$

methanol

methanol

ethanol

methanol

ethanol
ethanol

methanol:acetone:water

$(3 / 1 / 1, v / v / v)$

methanol

methano

ethanol

methanol

methanol:acetone:water

$(3 / 1 / 1, v / v / v)$

ethyl acetate

ethyl acetate

95\% aqueous ethano

$95 \%$ aqueous ethanol
$95 \%$ aqueous ethanol

aqueous eth
ethanol

methanol:acetone:water

$(3 / 1 / 1, v / v / v)$

methanol:acetone:water

$(3 / 1 / 1, v / v / v)$

$20 \%$ aqueous methanol

methanol:acetone:water

$(3 / 1 / 1, v / v / v)$
methanol

$20 \%$ aqueous methanol

$20 \%$ aqueous methano

$20 \%$ aqueous methano

$20 \%$ aqueous methanol

ethanol $20 \%$ aqueous methanol 
Table 2. Cont

\begin{tabular}{|c|c|c|c|c|}
\hline Compounds & Scorzonera Species & Concentration & Type of Solvent & References \\
\hline $\begin{array}{c}\text { Chlorogenic acid methyl ester } \\
\text { Cryptochlorogenic acid } \\
\text { Dihydrocaffeic acid } \\
\text { Dihydrocaffeic acid ethyl ester (ethyl dihydrocaffeate) } \\
\text { Dihydrocaffeic acid methyl ester (methyl dihydrocaffeate) } \\
\text { Dihydrocaffeic acid n-butyl ester (propyl dihydrocaffeate) } \\
\text { Hydrangeic acid 4'-O- } \beta \text { - } \beta \text {-D-glucopyenyld)dihydrofuran-2-one } \\
\text { Methyl 3-O-feruloylquinate } \\
\text { Protocatechuic acid } \\
\text { E-3-(3,4-dihydrosybenzylide } \\
\text { Z-3-(3,4-dihydroxybenzylidene)-5-(3,4-dihydroxyphenyl)-2(3H)-furanone }\end{array}$ & $\begin{array}{l}\text { S. hieraciifolia } \\
\text { S. latifolia } \\
\text { S. pygmaea } \\
\text { S. veratrifolia } \\
\text { S. veratrifolia } \\
\text { S. divaricata } \\
\text { S. divaricata } \\
\text { S. divaricata } \\
\text { S. divaricata } \\
\text { S. judaica } \\
\text { S. judaica } \\
\text { S. divaricata } \\
\text { S. cana var. jacquiniana } \\
\text { S. judaica }\end{array}$ & $\begin{array}{c}\mathrm{N} / \mathrm{D} \\
3.75 \mu \mathrm{g} / \mathrm{g} \\
10 \mu \mathrm{g} / \mathrm{g} \\
31.667 \mu \mathrm{g} / \mathrm{g} \\
11 \mu \mathrm{g} / \mathrm{g} \\
1.091 \mu \mathrm{g} / \mathrm{g} \\
1.091 \mu \mathrm{g} / \mathrm{g} \\
0.909 \mu \mathrm{g} / \mathrm{g} \\
7.273 \mu \mathrm{g} / \mathrm{g} \\
9.714 \mu \mathrm{g} / \mathrm{g} \\
3.857 \mu \mathrm{g} / \mathrm{g} \\
0.727 \mu \mathrm{g} / \mathrm{g} \\
8.5 \mu \mathrm{g} / \mathrm{g} \\
4.571 \mu \mathrm{g} / \mathrm{g}\end{array}$ & $\begin{array}{c}\text { ethanol } \\
\text { methanol } \\
\text { ethanol } \\
\text { methanol } \\
\text { methanol } \\
95 \% \text { aqueous ethanol } \\
95 \% \text { aqueous ethanol } \\
95 \% \text { aqueous ethanol } \\
95 \% \text { aqueous ethanol } \\
\text { chloroform:methanol (9:1) } \\
\text { methanol } \\
\text { 95\% aqueous ethanol } \\
\text { methanol } \\
\text { chloroform:methanol (9:1) }\end{array}$ & $\begin{array}{l}{[8]} \\
{[67]} \\
{[35]} \\
{[64]} \\
{[64]} \\
{[57]} \\
{[57]} \\
{[57]} \\
{[57]} \\
{[25]} \\
{[25]} \\
{[58]} \\
{[72]} \\
{[25]}\end{array}$ \\
\hline \multicolumn{5}{|l|}{ Phtalides } \\
\hline $\begin{array}{l}\text { (3RS)-3-[(SR)-hydroxy(4-hydroxyphenyl)-methyl]-7-methoxy-2-benzofuran-1(3H)-one } \\
(( \pm) \text {-scorzophthalide) }\end{array}$ & S. tomentosa & $7.538 \mu \mathrm{g} / \mathrm{g}$ & methanol & [36] \\
\hline $\begin{array}{c}( \pm) \text {-hydramacrophyllol A } \\
( \pm) \text {-hydramacrophyllol B } \\
( \pm) \text {-3-(4-hydroxybenzyl)-7-hydroxyphthalide (scorzoveratrin) } \\
\text { Scorzoveratrin } 4 \text {-O- } \beta \text {-glucoside } \\
\text { 3-(4- } \beta \text {-glucopyranosyloxybenzyl)-7-methoxyphthalide (scorzoveratrozit) }\end{array}$ & $\begin{array}{l}\text { S. judaica } \\
\text { S. tomentosa } \\
\text { S. tomentosa } \\
\text { S. judaica } \\
\text { S. latifolia } \\
\text { S. veratrifolia } \\
\text { S. latifolia } \\
\text { S. latifolia } \\
\text { S. veratrifolia }\end{array}$ & $\begin{array}{l}3.571 \mu \mathrm{g} / \mathrm{g} \\
31.91 \mu \mathrm{g} / \mathrm{g} \\
43.97 \mu \mathrm{g} / \mathrm{g} \\
6.429 \mu \mathrm{g} / \mathrm{g} \\
25.83 \mu \mathrm{g} / \mathrm{g} \\
65 \mu \mathrm{g} / \mathrm{g} \\
8.833 \mu \mathrm{g} / \mathrm{g} \\
50 \mu \mathrm{g} / \mathrm{g} \\
333.333 \mu \mathrm{g} / \mathrm{g}\end{array}$ & $\begin{array}{l}\text { chloroform:methanol (9:1) } \\
\text { methanol } \\
\text { methanol } \\
\text { chloroform:methanol (9:1) } \\
\text { methanol } \\
\text { methanol } \\
\text { methanol } \\
\text { methanol } \\
\text { methanol }\end{array}$ & $\begin{array}{l}{[25]} \\
{[36]} \\
{[36]} \\
{[25]} \\
{[67]} \\
{[64]} \\
{[67]} \\
{[67]} \\
{[64]}\end{array}$ \\
\hline \multicolumn{5}{|l|}{ Polysaccharides } \\
\hline Inulin & S. hispanica & $226.4 \mu \mathrm{g} / \mathrm{g}$ & water & [60] \\
\hline \multicolumn{5}{|l|}{ Sesquiterpene lactones } \\
\hline $\begin{array}{l}\text { (3aS,6aR,8S,9aR,9bS)-3,6,9-trimethylidene-8-[(2R,3R,4S,5S,6R)-3,4,5-trihydroxy-6- } \\
\text { (hydroxymethyl)oxan-2-yl]oxy-3a,4,5,6a,7,8,9a,9b-octahydroazuleno[4,5-b]furan-2-one } \\
\text { (glucozaluzanin C) }\end{array}$ & S. austriaca & $\mathrm{N} / \mathrm{D}$ & acetone & [33] \\
\hline $\begin{array}{c}(3 a S, 6 a R, 8 S, 9 a R, 9 b S) \text {-8-hydroxy-3,6,9-trimethylidene- } 3 a, 4,5,6 a, 7,8,9 a, 9 b \text {-octahydroazuleno[4,5-b]furan- } \\
\text { 2-one (zaluzanin } \\
\text { C) }\end{array}$ & S. austriaca & $\mathrm{N} / \mathrm{D}$ & acetone & [33] \\
\hline $\begin{array}{l}\text { (3aS,6aR,9aR,9bS)-3,6,9-trimethylidene-3a,4,5,6a,7,8,9a,9b-octahydroazuleno[4,5-b]furan-2-one } \\
\text { (dehydrocostus lactone) }\end{array}$ & S. austriaca & $\mathrm{N} / \mathrm{D}$ & acetone & [33] \\
\hline $\begin{array}{c}\text { 11 } 1,13 \text {-dihydrozaluzanin C } \\
\text { 14-isovaleroxyscorzoaustricin } \\
\text { 14-isovaleroxyscorzoaustricin sulfate } \\
\text { 4-epi-dihydroestafiatol } \\
\text { Biguaiascorzolide A } \\
\text { Biguaiascorzolide B } \\
\text { Diacetoxyisolippidiol }\end{array}$ & $\begin{array}{l}\text { S. austriaca } \\
\text { S. austriaca } \\
\text { S. austriaca } \\
\text { S. austriaca } \\
\text { S. austriaca } \\
\text { S. austriaca } \\
\text { S. austriaca }\end{array}$ & $\begin{array}{l}\mathrm{N} / \mathrm{D} \\
4.286 \mu \mathrm{g} / \mathrm{g} \\
7.143 \mu \mathrm{g} / \mathrm{g} \\
4.286 \mu \mathrm{g} / \mathrm{g} \\
5.714 \mu \mathrm{g} / \mathrm{g} \\
0.857 \mu \mathrm{g} / \mathrm{g} \\
\mathrm{N} / \mathrm{D}\end{array}$ & $\begin{array}{l}\text { acetone } \\
\text { acetone } \\
\text { acetone } \\
\text { acetone } \\
\text { acetone } \\
\text { acetone } \\
\text { acetone }\end{array}$ & $\begin{array}{l}{[33]} \\
{[33]} \\
{[33]} \\
{[33]} \\
{[17]} \\
{[17]} \\
{[33]}\end{array}$ \\
\hline
\end{tabular}


Table 2. Cont

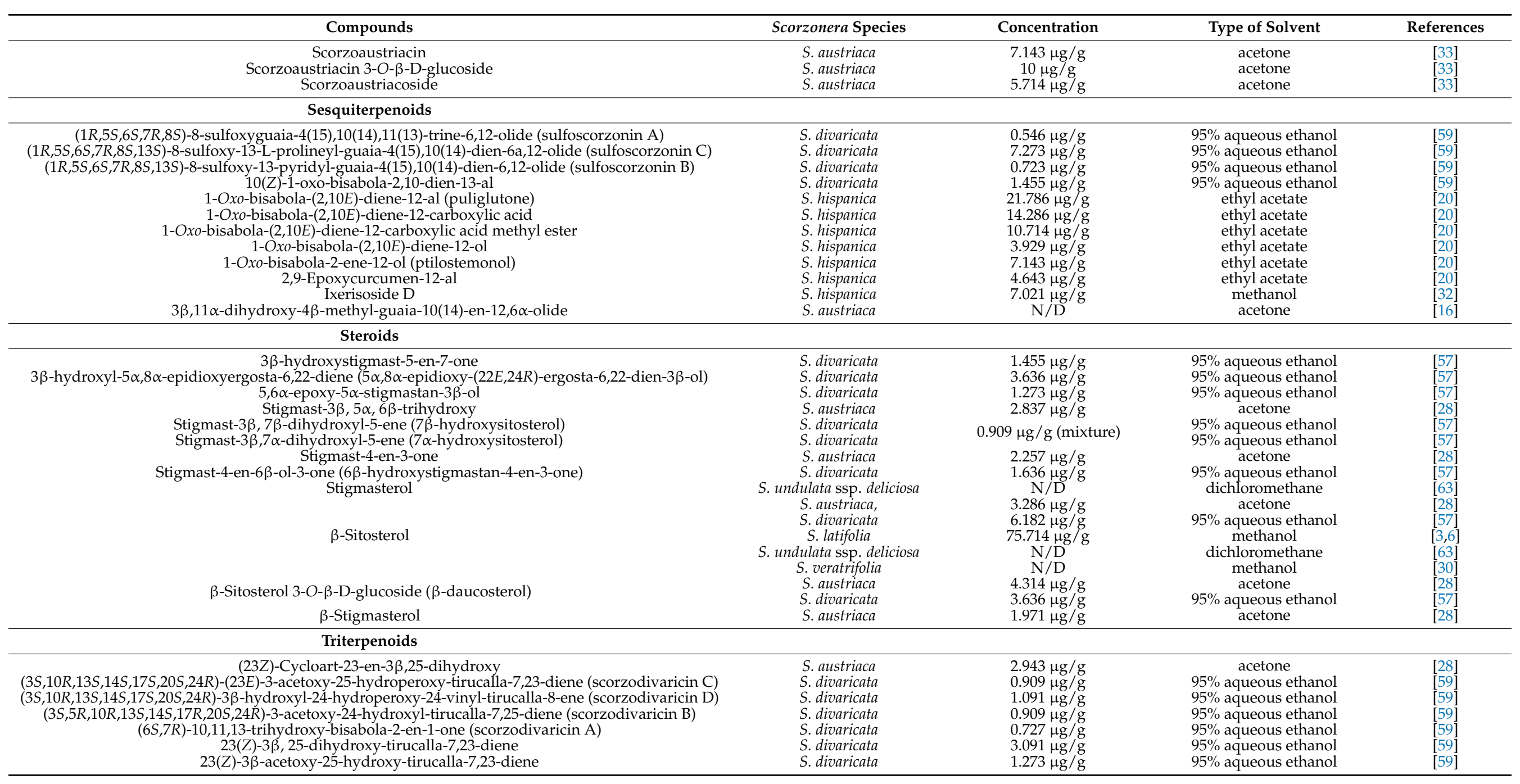


Table 2. Cont

\section{Compounds}

$3 \alpha$-hydroxyolean-5-ene

$3 \beta$-acetoxyglutin-5(10)-en-6-oxo

$3 \beta$-acetyl-11 $\alpha, 12 \alpha$-oxidotaraxerol

3- $\beta$-hydroxy-fern-7-en-6-one-acetate

3- $\beta$-hydroxy-fern-8-en-7-one-acetate $9 \beta, 19$-cyclolanostane- 24 -en-3-oxo

Amyrin $\beta$-acetate 4-en-3 $\beta$-acetyl-11 $\alpha, 12 \alpha$-epoxy Fern-7-en-3-one
-

Fern-7-en-3- $\beta$-one

Germanicol acetate

Germanicone

Glutinol

Lupenone

Lupeol
Scorzonera Species

S. aristata

S. austriaca

S. austriaca

S. cinerea
S. eriophora

S. latifolia

S. sublanata

S. tomentosa
S. latifolia

S. Laustrifiaca

S. undulata ssp. deliciosa

S. austriaca

S. veratrifolia
S. latifolia

S. latitifolia

S. veratrifolia

S. veratrifolia,

S. veratrifolia

S. austriaca

S. acuminata

S. aristata

S. austriaca

S. cana var. jacquiniana S. cinerea S. eriophora
S. incisa

S. laciniata ssp. laciniata S. latifolia

S. mollis ssp. szowitsii

S. parviflora

S. suberosa ssp. suberosa S. sublanata S. tomentosa
Concentration

Type of Solvent

References

$$
\begin{gathered}
19.036 \mu \mathrm{g} / \mathrm{g} \\
4.086 \mu \mathrm{g} / \mathrm{g} \\
3.3171 \mu \mathrm{g} / \mathrm{g} \\
65 \pm 1 \mu \mathrm{g} / \mathrm{g} \\
20 \pm 1 \mu \mathrm{g} / \mathrm{g} \\
50 \pm 1 \mu \mathrm{g} / \mathrm{g} \\
7.143 \mu \mathrm{g} / \mathrm{g} \\
35 \pm 1 \mu \mathrm{g} / \mathrm{g} \\
47 \pm 1 \mu \mathrm{g} / \mathrm{g} \\
\mathrm{N} / \mathrm{D} \\
7.314 \mu \mathrm{g} / \mathrm{g} \\
10.103 \mu \mathrm{g} / \mathrm{g} \\
2.229 \mu \mathrm{g} / \mathrm{g} \\
\mathrm{N} / \mathrm{D} \\
6.714 \mu \mathrm{g} / \mathrm{g} \\
32.143 \mu \mathrm{g} / \mathrm{g} \\
\mathrm{N} / \mathrm{D} \\
\mathrm{N} / \mathrm{D} \\
\mathrm{N} / \mathrm{D} \\
4.4861 \mu \mathrm{g} / \mathrm{g} \\
\mathrm{N} / \mathrm{D} \\
512 \pm 1 \mu \mathrm{g} / \mathrm{g} \\
\mathrm{N} / \mathrm{D} \\
\mathrm{N}
\end{gathered}
$$

$2.7431 \mu \mathrm{g} / \mathrm{g}$

$932 \pm 2 \mu \mathrm{g} / \mathrm{g}$

$1073 \pm 6 \mu \mathrm{g} / \mathrm{g}$

$244 \pm 7 \mu \mathrm{g} / \mathrm{g}$

$447 \pm 2 \mu \mathrm{g} / \mathrm{g}$

$447 \pm 2 \mu \mathrm{g} / \mathrm{g}$

$224 \pm 1 \mu \mathrm{g} / \mathrm{g}$

$282 \pm 11 \mu \mathrm{g} / \mathrm{g}$

$132 \pm 4 \mu \mathrm{g} / \mathrm{g}$

$342 \pm 4 \mu \mathrm{g} / \mathrm{g}$

$415 \pm 1 \mu \mathrm{g} / \mathrm{g}$

$564 \pm 2 \mu \mathrm{g} / \mathrm{g}$ methanol:acetone:water

$(3 / 1 / 1, v / v / v)$

[56]

acetone

acetone

$n$-hexane

n-hexane

n-hexane

methanol

n-hexane

methanol

methano

dichloromethane

acetone

methano

methano

methano

methano

methano

acetone

methanol

$n$-hexane

methanol:acetone:wate

$(3 / 1 / 1, v / v / v)$

methanol

acetone
$n$-hexane

$n$-hexane

$n$-hexane

$n$-hexane

$n$-hexane

$n$-hexane
$n$-hexane

$n$-hexane

$n$-hexane

$n$-hexane

$n$-hexane

n-hexane

n-hexane

$n$-hexane
methanol 
Table 2. Cont.

Compounds

Lupeol acetate

Magnificol

Methyl oleanate

Olean-12-en-11-one-3-acetyl

Oleanolic acid

Taraxasterol

Taraxasterol acetate/Taraxasteryl acetate

Taraxasteryl myristate

Urs-12-en-11-one-3-acetyl

$\alpha$-Amyrin

\begin{tabular}{|c|c|c|c|}
\hline Scorzonera Species & Concentration & Type of Solvent & References \\
\hline S. acuminata & $297 \pm 1 \mu \mathrm{g} / \mathrm{g}$ & $n$-hexane & [27] \\
\hline S. cana var. jacquiniana & $4273 \pm 12 \mu \mathrm{g} / \mathrm{g}$ & $n$-hexane & [27] \\
\hline S. cinerea & $3645 \pm 8 \mu \mathrm{g} / \mathrm{g}$ & $n$-hexane & [27] \\
\hline S. eriophora & $2195 \pm 7 \mu \mathrm{g} / \mathrm{g}$ & $n$-hexane & [27] \\
\hline S. incisa & $736 \pm 10 \mu \mathrm{g} / \mathrm{g}$ & $n$-hexane & [27] \\
\hline S. laciniata ssp. laciniata & $3212 \pm 13 \mu \mathrm{g} / \mathrm{g}$ & $n$-hexane & [27] \\
\hline S. latifolia & $2261 \pm 94 \mu \mathrm{g} / \mathrm{g}$ & $n$-hexane & [27] \\
\hline S. mirabilis & $1356 \pm 2 \mu \mathrm{g} / \mathrm{g}$ & $n$-hexane & [27] \\
\hline S. mollis ssp. szowitsii & $1244 \pm 1 \mu \mathrm{g} / \mathrm{g}$ & $n$-hexane & [27] \\
\hline S. parviflora & $711 \pm 3 \mu \mathrm{g} / \mathrm{g}$ & $n$-hexane & [27] \\
\hline S. suberosa ssp. suberosa & $1261 \pm 5 \mu \mathrm{g} / \mathrm{g}$ & $n$-hexane & [27] \\
\hline S. sublanata & $3920 \pm 8 \mu \mathrm{g} / \mathrm{g}$ & $n$-hexane & [27] \\
\hline S. tomentosa & $2502 \pm 7 \mu \mathrm{g} / \mathrm{g}$ & $n$-hexane & [27] \\
\hline S. veratrifolia & $\mathrm{N} / \mathrm{D}$ & methanol & [30] \\
\hline S. aristata & $\mathrm{N} / \mathrm{D}$ & $\begin{array}{c}\text { methanol:acetone:water } \\
(3 / 1 / 1, v / v / v)\end{array}$ & [56] \\
\hline S. undulata ssp. deliciosa & N/D & $\begin{array}{l}\text { methanol } \\
\text { dichloromethane }\end{array}$ & [63] \\
\hline S. undulata ssp. deliciosa & $\mathrm{N} / \mathrm{D}$ & dichloromethane & [63] \\
\hline S. cinerea & $115 \pm 1 \mu \mathrm{g} / \mathrm{g}$ & $n$-hexane & [27] \\
\hline S. incisa & $151 \pm 1 \mu \mathrm{g} / \mathrm{g}$ & $n$-hexane & [27] \\
\hline S. latifolia & $8 \mu \mathrm{g} / \mathrm{g}$ & methanol & [47] \\
\hline & $135 \pm 1 \mu \mathrm{g} / \mathrm{g}$ & $n$-hexane & [27] \\
\hline S. tomentosa & $187 \pm 1 \mu \mathrm{g} / \mathrm{g}$ & $n$-hexane & [27] \\
\hline S. divaricata & $1.818 \mu \mathrm{g} / \mathrm{g}$ & $95 \%$ aqueous ethanol & [59] \\
\hline S. austriaca & $3.371 \mu \mathrm{g} / \mathrm{g}$ & acetone & [28] \\
\hline S. veratrifolia & N/D & methanol & [30] \\
\hline S. cana var. jacquiniana & $719 \pm 3 \mu \mathrm{g} / \mathrm{g}$ & $n$-hexane & [27] \\
\hline S. cinerea & $2171 \pm 6 \mu \mathrm{g} / \mathrm{g}$ & $n$-hexane & [27] \\
\hline S. eriophora & $3212 \pm 17 \mu \mathrm{g} / \mathrm{g}$ & $n$-hexane & [27] \\
\hline S. incisa & $1191 \pm 5 \mu \mathrm{g} / \mathrm{g}$ & $n$-hexane & [27] \\
\hline S. laciniata ssp. laciniata & $276 \pm 3 \mu \mathrm{g} / \mathrm{g}$ & $n$-hexane & [27] \\
\hline S. latifolia, & $4201 \pm 16 \mu \mathrm{g} / \mathrm{g}$ & $n$-hexane & [27] \\
\hline S. mirabilis & $2099 \pm 4 \mu \mathrm{g} / \mathrm{g}$ & $n$-hexane & [27] \\
\hline S. mollis ssp. szowitsii & $3791 \pm 14 \mu \mathrm{g} / \mathrm{g}$ & n-hexane & [27] \\
\hline S. parviflora & $811.96 \pm 4 \mu \mathrm{g} / \mathrm{g}$ & $n$-hexane & [27] \\
\hline S. suberosa ssp. suberosa & $2340 \pm 6 \mu \mathrm{g} / \mathrm{g}$ & $n$-hexane & [27] \\
\hline S. sublanata & $4981 \pm 2 \mu \mathrm{g} / \mathrm{g}$ & $n$-hexane & [27] \\
\hline S. tomentosa & $3168 \pm 12 \mu \mathrm{g} / \mathrm{g}$ & $n$-hexane & [27] \\
\hline S. veratrifolia & N/D & methanol & [30] \\
\hline S. latifolia & $142.85 \mu \mathrm{g} / \mathrm{g}$ & methanol & [6] \\
\hline S. latifolia & N/D & methanol & [10] \\
\hline S. acuminata & $1646 \pm 10 \mu \mathrm{g} / \mathrm{g}$ & $n$-hexane & [27] \\
\hline S. cana var. jacquiniana & $920 \pm 11 \mu \mathrm{g} / \mathrm{g}$ & n-hexane & [27] \\
\hline S. cinerea & $3221 \pm 13 \mu \mathrm{g} / \mathrm{g}$ & $n$-hexane & [27] \\
\hline S. laciniata ssp. laciniata & $146 \pm 4 \mu \mathrm{g} / \mathrm{g}$ & n-hexane & [27] \\
\hline S. mollis ssp. szowitsii & $609 \pm 6 \mu \mathrm{g} / \mathrm{g}$ & $n$-hexane & [27] \\
\hline S. tomentosa & $969 \pm 11 \mu \mathrm{g} / \mathrm{g}$ & $n$-hexane & [27] \\
\hline
\end{tabular}


Table 2. Cont.

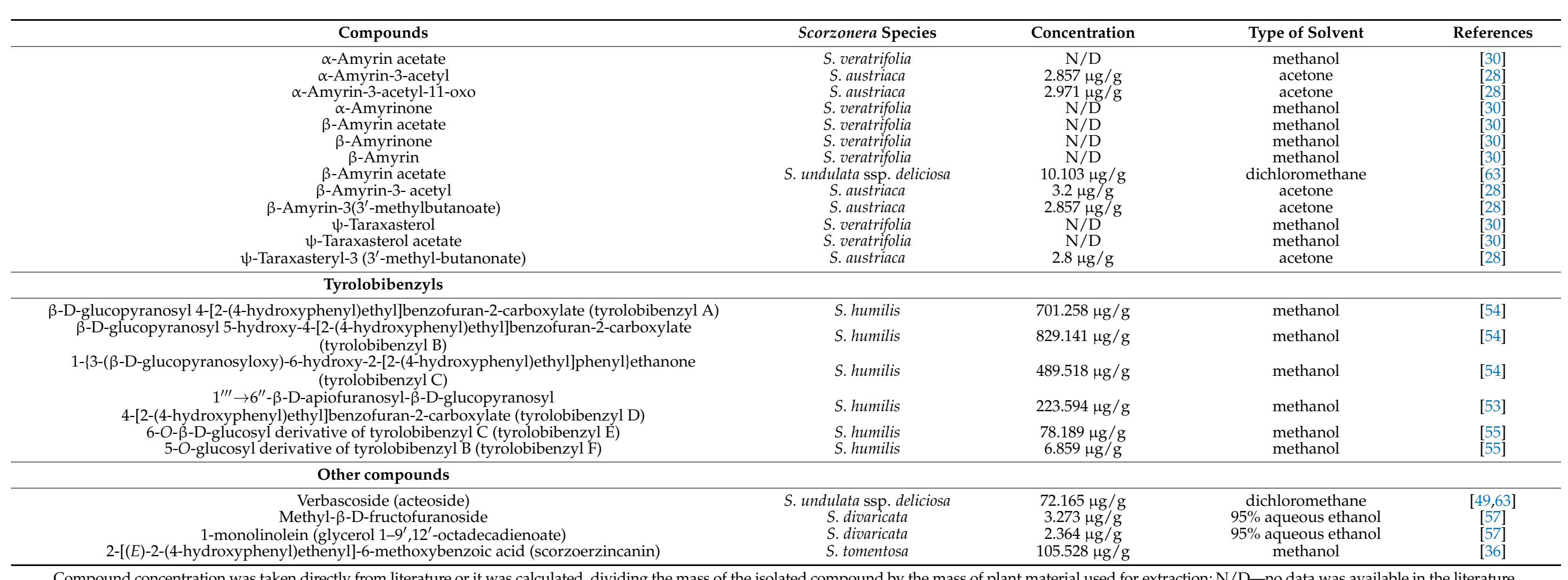




Table 3. Compounds isolated from Scorzonera whole plants.

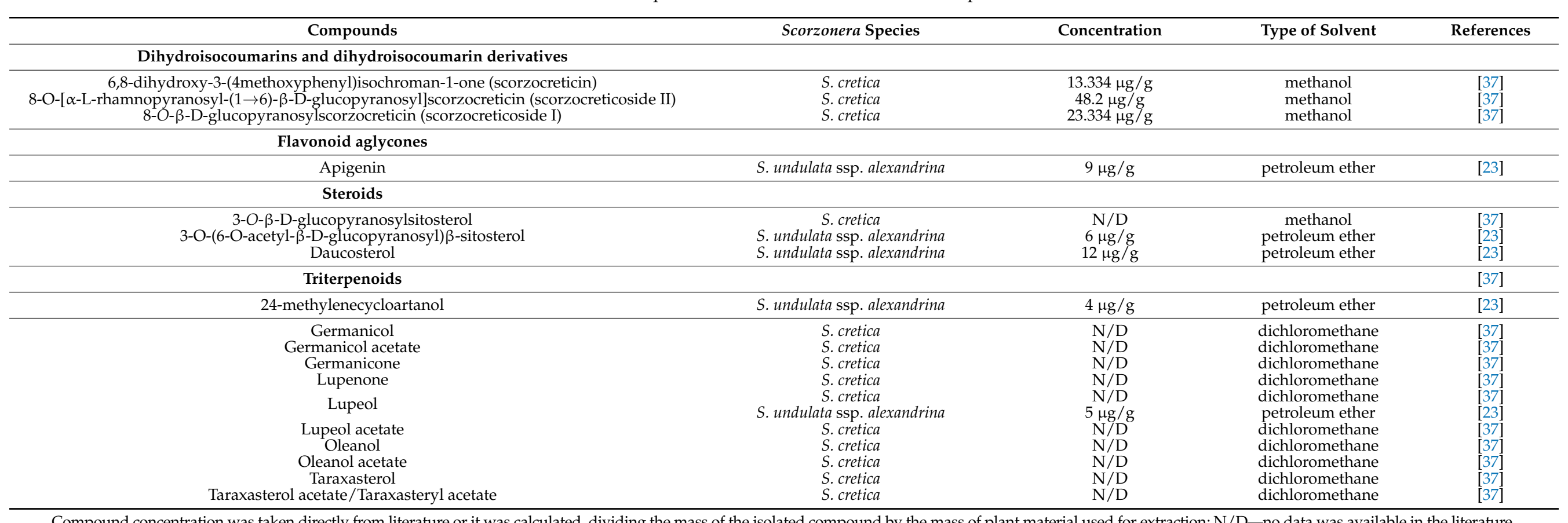






\section{Biological Activity}

The biological activity of species within the Scorzonera genus is the subject of research due to their presence in folk medicine in Eurasia and northern Africa. In Mongolia, S. divaricata and S. pseudodivaricata play a significant role in herbal therapy. S. divaricata is used to treat fever and poisonous ulcers or even malignant stomach neoplasia. S. pseudodivaricata is a folk remedy for digestive problems, parasites, or lung edema [14].

Species that belong to the Scorzonera genus are reported to be the source of numerous bioactive compounds. Researchers evaluate their potential as antioxidant [58,59,62,74], anti-inflammatory $[27,68,75]$, and pain-relieving agents [6,70], as well as their cytotoxicity against cancer cell lines $[20,28,59]$ and wound healing properties $[4,5]$. Biological activities of Scorzonera species in vitro are summarized in Figure 1.
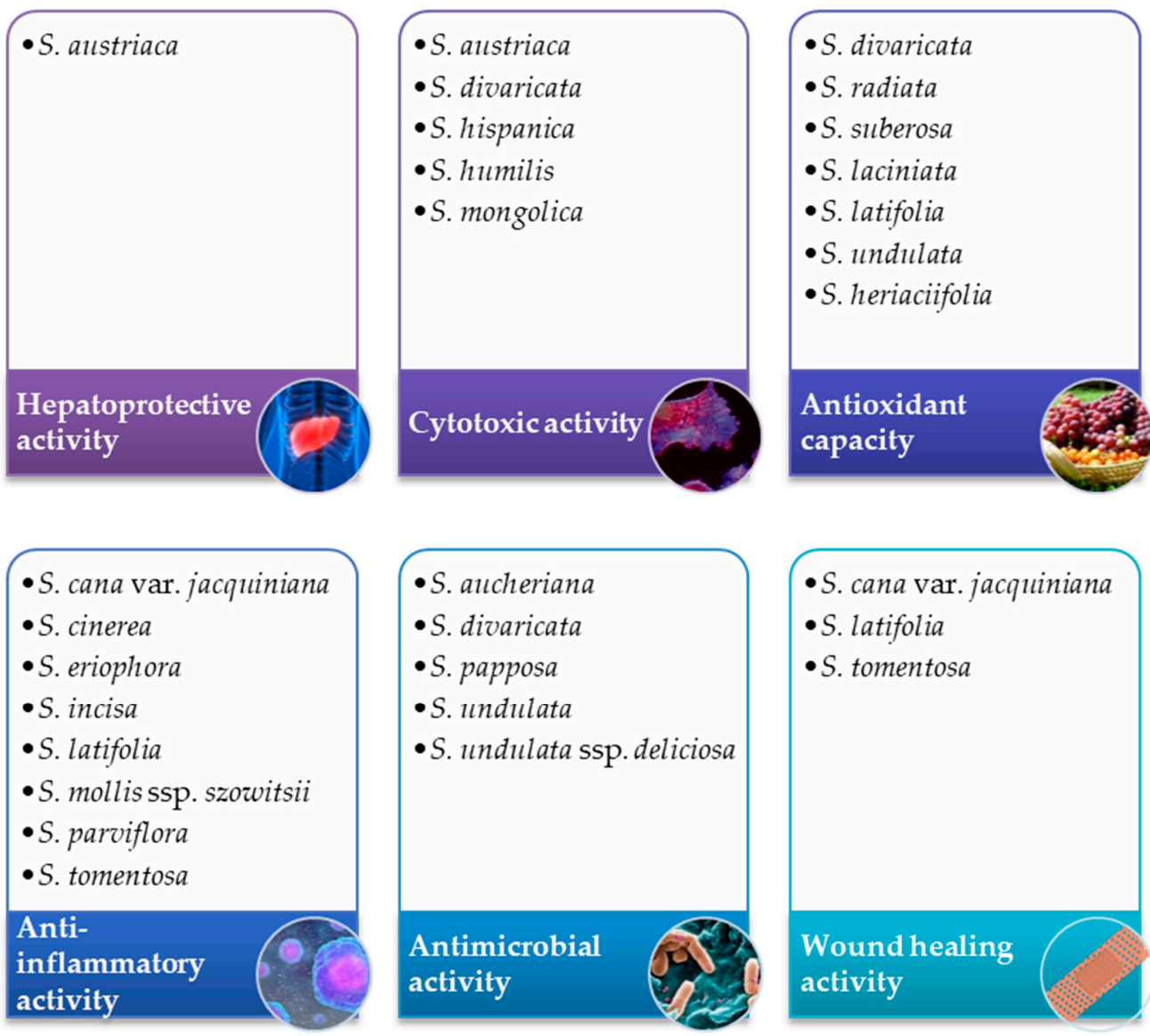

Figure 1. Biological activity of species within the genus Scorzonera evaluated in vitro. Species were assigned to sections according to their activity investigated in in vitro tests, described in Sections 4.1-4.7.

\subsection{Cytotoxic Activity}

Cytotoxicity is the primary characteristic of compounds and substances in terms of their qualification as therapeutic agents and is the toxicity that a certain factor causes in live cells [76]. High cytotoxicity against rapidly dividing cancer cells in vitro is the basis for further research on their bioactivity (e.g., necrosis, autophagy, or apoptosis induction), low cytotoxicity on the other hand is desired in the development of drugs that are not intended to induce death in cells. In this review, the cytotoxicity of extracts and compounds obtained and isolated from Scorzonera species against cancer cell lines was presented.

The first reported attempt to evaluate the antineoplastic activity of Scorzonera species in vitro was made in 2000 by Zidorn and colleagues [54]. The biological activity of compounds isolated from a methanol extract from S. humilis subaerial parts (together with newly isolated tyrolobibenzyls) was assessed and none influenced the DNA biosynthesis 
in the GTB and HL60 human leukemia cell lines at the concentration range of $0.25-4.00 \mu \mathrm{M}$. In their further research, tyrolobibenzyls D was isolated from the extract. It was assayed for cytotoxicity against the P388 (mouse leukemia) cell line along with previously obtained tyrolobibenzyls A-C and their peracetyl derivatives. In the assay, only Tylorobibenzyl $\mathrm{D}$ exhibited low cytotoxic activity with $\mathrm{IC}_{50}$ (half-maximal inhibitory concentration) of $25 \mu \mathrm{g} / \mathrm{mL}$. The cytotoxicity of crude extracts was assayed as well and no activity was observed up to the point where the concentration reached $0.5 \mathrm{mg} / \mathrm{mL}$. The EtOAc fraction of the crude extract exhibited cytotoxic properties with $\mathrm{IC}_{50}$ value at the concentration of $95 \mu \mathrm{g} / \mathrm{mL}$. In the research, the antimicrobial activity of tyrolobibenzyls and their derivatives was evaluated but none was active against neither bacteria nor fungi. The DPPH assay did not reveal any significant radical scavenging properties of tyrolobibenzyls and the compounds were able to inhibit the activity of COX-1 (cyclooxygenase 1) to an insignificant degree [53].

In the study from 2009, Wang and colleagues [31] obtained two triterpene fatty esters: erythrodiol and $3 \beta$-tetradecanoyl erythrodiol from a methanol extract of Scorzonera mongolica whole plants. The isolated compounds were then assayed for their cytotoxicity towards 3 cancer cell lines (P388 mouse leukemia cell line, A549 human lung cancer cell line, and Bel-7402 human hepatocellular carcinoma cell line) and both esters exhibited cytotoxic activity against A549 lung cancer cells (in the concentration of $50 \mu \mathrm{g} / \mathrm{mL}$, the compounds induced the cell growth inhibition by $66.8 \%$ and $69.8 \%$ ).

Two out of five congeners (Scorzodihydrostilbenes A and B) isolated from the methanol extract from the aerial parts of Scorzonera radiata Fisch. were tested in the MTT cytotoxicity assay but at a concentration of $10 \mu \mathrm{g} / \mathrm{mL}$, neither displayed cytotoxic activity toward mouse lymphoma cell line (L5175Y) [15]. The concentration $(10 \mu \mathrm{g} / \mathrm{mL})$ converted to $\mu \mathrm{M}$ is $21.53 \mu \mathrm{M}$ for Scorzodihydrostilbene A and $20.90 \mu \mathrm{M}$ for Scorzodihydrostilbene B. When compared to a study from 2007, dihydrostilbenes isolated from a Bulbophyllum odoratissimum Lindl., low toxicity of Scorzonera radiata Fisch. is even more notable, compounds in the mentioned study were toxic towards SGC-7901 (human gastric cancer), KB (nasopharyngeal carcinoma), and HT-1080 (fibrosarcoma) cell lines with $\mathrm{IC}_{50}$ values of 5.50-9.20 $\mu \mathrm{M}$ for SGC-7901 and KB lines and 25.50-40 $\mu \mathrm{M}$ for HT-1080 line [77].

A screening study on cytotoxic activity of several species of Asteraceae genus present in Hungary, including Scorzonera austriaca Willd, was carried out in 2009. In a cytotoxicity assay, the most active against human cell lines: A431 (skin epidermoid carcinoma), HeLa (cervix adenocarcinoma), and MCF-7 (breast adenocarcinoma) was a chloroform root extract of $S$. austriaca $\mathrm{IC}_{50}$ values of the extract were: $4.71 \mu \mathrm{g} / \mathrm{mL}$ for A431 line, $6.42 \mu \mathrm{g} / \mathrm{mL}$ for HeLa line and $5.52 \mu \mathrm{g} / \mathrm{mL}$ for MCF-7 line. It was more active than the extracts obtained using other solvents, as well as leaf extracts from the same plant-at a concentration of $10 \mu \mathrm{g} / \mathrm{mL}$ the antiproliferative activity of $S$. austriaca root chloroform was at $86.32 \%$ for A431 cell line, $77.27 \%$ for HeLa cell line, and $83.79 \%$ for MCF-7 cell line. Other S. austriaca extracts obtained in the study did not influence the proliferation of those cell lines by more than $48.11 \%$ [78]. Those results can be compared to a 2018 research, in which leaf chloroform extract from another species within the Asteraceae family, Anvillea garcinii (Burm.f.) DC., exhibited antiproliferative properties against MCF-7 and HeLa cell lines with $\mathrm{IC}_{50}$ of $24.50 \mu \mathrm{g} / \mathrm{mL}$ for MCF-7 and $12 \mu \mathrm{g} / \mathrm{mL}$ for HeLa [79]. Another Asteraceae family member, Pulicaria undulata (Forssk.) Oliver., was evaluated as a potential source of cytotoxic agents. Whole plant chloroform extract turned out to have cytotoxic properties with the IC $_{50}$ value of $16.4 \mu \mathrm{g} / \mathrm{mL}$ for MCF-7 cell line, $3.01 \mu \mathrm{g} / \mathrm{mL}$ for HepG2, and $7.4 \mu \mathrm{g} / \mathrm{mL}$ for HCT-116 cell lines. Those values were compared to cisplatin used as a positive control in the study $\left(\mathrm{IC}_{50}\right.$ of cisplatin was 3.68-4.51 $\mathrm{g} / \mathrm{mL}$ ) [80]. However, in the study from 2011, Bader et al. isolated nine new phenolic compounds and nine known phenolic derivatives from Scorzonera judaica root extracts. The newly obtained compounds were assayed for their cytotoxic activity toward human lymphocyte T cells, as well as MCF-7 and HeLa cell lines. Compounds did not exhibit cytotoxic activity in concentrations below $100 \mu \mathrm{M}$ [25]. 
Zhu and colleagues [17] discovered that two new dimeric guaianolides (biguaiascorzolides A and B) are present in an acetone extract from Scorzonera austriaca roots. In the study, biguaianoscorzolide A was acetylated and the derivative's cytotoxicity against adriamycin-resistant myelogenous leukemia (K562/ADM) cell line and gastric carcinoma (BGC-823) cell line was measured. The compound's activity towards K562/ADM cells $\left(\mathrm{IC}_{50}=39.8 \mu \mathrm{M}\right)$ was more significant than towards the MGC-803 cell line $\left(\mathrm{IC}_{50}>100 \mu \mathrm{M}\right)$, which suggests that the compound's cytotoxic activity might depend on the type of tumor cells. In a continuation of the study, Zhu and colleagues [33] elucidated the presence of six sesquiterpene lactones in acetone and ethanol S. austriaca root extracts (scorzoaustriacoside, scorzoaustriacin, scorzoaustriacin 3-O- $\beta$-D-glucoside, 4-epi-dihydroestafiatol, 14-isovaleroxyscorzoaustricin, and 14-isovaleroxyscorzoaustricin sulfate). The cytotoxic activity against four cancer cell lines (K562, K562/ADM, BGC-823, and Hep-G2) of scorzoaustriacin, scorzoaustriacin 3-O- $\beta$-D-glucoside, 14 -isovaleroxyscorzoaustricin, and 14isovaleroxyscorzoaustricin sulfate was assayed and only scorzoaustriacin was reported cytotoxic towards K562 (human myelogenous leukemia) cell line $\left(\mathrm{IC}_{50}=11.3 \mu \mathrm{M}\right)$.

In the study by Granica et al. [20], (-)-syringaresinol was isolated from the ethyl acetate extract of Scorzonera hispanica subaerial parts. It was then reported that (-)-syringaresinol was cytotoxic towards NCI and MMS- 1 myeloma cell lines and exhibited moderate activity against SW-480 colon cancer cells. Moreover, the compound's cytotoxicity was reported in peripheral blood mononuclear cells. In the previous research on (-)-syringaresinol, it was reported to exhibit the ability to induce apoptosis and arrest the $G_{1}$ phase in the HL-60 human leukemia cell line [81]. Jeong et al. [82] found out that (-)-syringaresinol inhibits P-glycoprotein in MCF-7/ADR human breast cancer cell line and enhances the cytotoxic activity of daunomycin.

In a phytochemical study on Scorzonera divaricata root ethanol extract, one of the isolated compounds, a tirucallane terpene $((3 S, 5 R, 10 R, 13 S, 14 S, 17 R, 20 S, 24 R)-3$-acetoxy-24hydroxyl-tirucalla-7,25-dienem, named scorzodivaricin B), exhibited a cytotoxic activity towards HeLa, HepG2, HL60 and SMMC-7721 (human cervical cancer, human liver cancer human leukemia cancer and hepatocellular carcinoma, accordingly) cell lines with $\mathrm{IC}_{50}$ values between $24.4 \pm 3.6 \mu \mathrm{M}$ and $66.7 \pm 5.2 \mu \mathrm{M}$. Cisplatin used as a positive control in the study exhibited higher activity with $\mathrm{IC}_{50}$ within the range of $7.45 \pm 0.9-12.8 \pm 2.4 \mu \mathrm{M}$ [59].

$\mathrm{Wu}$ and colleagues [34] evaluated five compounds isolated from Scorzonera divaricata aerial parts for their potential activity against cancer cell lines (HepG2, HeLa, and K562). Sulfoscorzonin E exhibited cytotoxicity similar to 5-FU. IC 50 values of sulfoscorzonin $\mathrm{E}$ were $4.21 \mu \mathrm{g} / \mathrm{mL}(10.59 \mu \mathrm{M})$ for HepG2, $8.15 \mu \mathrm{g} / \mathrm{mL}(20.5 \mu \mathrm{M})$ for HeLa, and $6.53 \mu \mathrm{g} / \mathrm{mL}$ $(16.43 \mu \mathrm{M})$ for K562 cell line. Sacrolide A was active against HeLa and HepG2 cells $\left(\mathrm{IC}_{50}=6.28 \mu \mathrm{g} / \mathrm{mL}=20.16 \mu \mathrm{M}\right.$ for HeLa and $3.56 \mu \mathrm{g} / \mathrm{mL}=11.43 \mu \mathrm{M}$ for HepG2 lines). In the study, sulfoscorzonin $\mathrm{D}$, glucozaluzanin $\mathrm{C}$, and $1 \beta, 4 \alpha$-dihydroxy- $5 \alpha, 6 \beta, 7 \alpha, 11 \beta H$ eudermn-12,6-olide did not exhibit any significant cytotoxic activity at the concentration below $80 \mu \mathrm{g} / \mathrm{mL}(141.92-298.32 \mu \mathrm{M})$.

\subsection{Anti-Inflammatory Activity}

For centuries, suppression of inflammatory response has been an observed effect of various medicinal plants. Ethnopharmacological reports give examples of plants extracts able to combat the process of inflammation in human bodies and; therefore, novel plantderived products are investigated for their anti-inflammatory activity [83].

A study carried out by Bahadır-Ackkara et al. in 2018 [27] revealed that $n$-hexane extracts from roots and aerial parts of eleven Scorzonera species (S. acuminata, S. cinerea, S. eriophora, S. incisa, S. latifolia, S. mirabilis, S. mollis ssp. szowitsii, S. parviflora, S. suberosa ssp. suberosa and $S$. tomentosa) contained significant amounts of triterpenes, including taraxasteryl acetate, lupeol, and lupeol acetate. In general, root extracts were notably richer in analyzed triterpenes, except for lupeol, whose concentration in aerial parts of S. incisa, $S$. latifolia, S. mirabilis, S. parviflora, and S. suberosa was approximately three to seven times higher than in root extracts (approximately $0.9-1.5 \mathrm{mg} / \mathrm{g}$ ) . The results from the study 
correlate with previously observed anti-inflammatory and pain-relieving properties of lupeol $[84,85]$.

The evaluation of the anti-inflammatory properties of Scorzonera pygmaea subaerial parts was conducted in 2018 by measuring COX (cyclooxygenase) inhibition. The inhibitory activity of ethanol extract and its fractions against COX-1 and COX-2 (cyclooxygenase 2) was low [35]. That is on the contrary to the study of Bahadır Ac1kara et al. from 2015 [10], where it has been observed that extracts from other Scorzonera species (S. cana var. jacquiniana, S. cinerea, S. eriophora, S. incisa, S. latifolia, S. mollis ssp. szowitsii, S. parviflora, and $S$. tomentosa) have an inhibitory effect on pro-inflammatory cytokines (TNF$\alpha$ (tumor necrosis factor $\alpha$ ) and IL-1 $\beta$ (interleukin $1 \beta)$ ) production and NF-kB (nuclear factor kappa B) nuclear translocation in macrophages. However, it might suggest that the anti-inflammatory activity of $S$. pygmaea could be evaluated by the measurement of the inhibitory activity against pro-inflammatory cytokines, as they induce COX production [35].

\subsection{Analgesic Activity}

Pain is an experience known to nearly every animal. There are cases where pain requires medical intervention. Although pain may indicate injury of organs, nervous systemderived pain should be relieved beforehand to prevent the deterioration of the quality of the patient's life. Morphine, a well-known analgesic, was isolated from opium 200 years ago [86]. Nowadays other natural products are assayed for their pain-relieving activity.

Scorzonera latifolia is a plant endemic to Turkey, whose roots are used as a pain-reducing and anthelmintic agent in Turkish folk medicine [4]. An in vivo study on the properties of a methanol extract from S. latifolia roots showed that $n$-hexane, chloroform, ethyl acetate, $n$-butanol, and water fractions indeed exhibit analgesic activity on mice in the dose of $50 \mathrm{mg} / \mathrm{kg}$ in the tail-flick test. Taraxasteryl mirystate and taraxasteryl acetate present in one of the extracts were active in the dose of $10 \mathrm{mg} / \mathrm{kg}$ in both writhing test and flick-tail test. The general antinociceptive properties of S. latifolia are reported to be significant. Such outcome of the study can be a result of the synergy of the extract's components [6]. The study was extended to four Scorzonera species in 2012. It was then reported that $S$. tomentosa, S. latifolia, and S. mollis ssp. szowitsii all possess analgesic properties in the writhing test and tail-flick test (the dose was $100 \mathrm{mg} / \mathrm{kg}$ ) [70].

\subsection{Hepatoprotective Activity}

The liver plays a significant role in the metabolism and detoxication of the human body. Because of its importance, liver diseases are one of the greatest threats to people's lives. Herbal medicine has used plants as preventive agents for hepatic problems for ages [87]. Based on that knowledge, in vitro and in vivo investigations are conducted to assess the hepatoprotective potential of plants, including the ones with the Scorzonera genus.

\subsubsection{In Vitro Assays}

A study from 2016 investigated the hepatoprotective properties of Scorzonera austriaca. The plant is used in folk medicine to treat hepatitis B in China. Xie et al. [24] isolated flavonoid glycosides and their derivatives from Scorzonera austriaca herb ethanolic extract. Having measured the concentration of ALT (alanine aminotransferase) in $\mathrm{CCl}_{4}$-treated rat hepatocytes, it was reported that two flavonoid glycoside derivatives, 5,7,41-trihydroxyflavone 8-C-(6"-O-trans-caffeoyl $\beta$-D-glucopyranoside) and 5,7,31,41-tetrahydroxyflavone 8-C-(6"-Otrans-caffeoyl $\beta$-D-glucopyranoside), present in herbs of $S$. austriaca have hepatoprotective properties. That conclusion confirmed the validity of the use of the plant in the treatment of hepatitis B in the traditional medicine of China.

\subsubsection{In Vivo Assays}

The in vivo assays of a Scorzonera alexandrina hydroethanolic extract from aerial and subaerial parts revealed that the extract caused a reduction in glucose concentration in 
rat's blood, as well as the ALT, and total protein levels in doses of 200 and $400 \mathrm{mg} / \mathrm{kg}$. The extract also exhibited hepatoprotective, and anti-ulcerogenic effects in rats [75].

Hepatoprotective activities of the roots of several Scorzonera species (S. cana var. jacquiniana, S. latifolia, S. mollis ssp. szowitsii, S. parviflora, S. tomentosa), together with compounds isolated from the $S$. latifolia root extract (chlorogenic acid, hydrangenol-8-O- $\beta$ glucoside, and scorzotomentosin- $4^{\prime}-\mathrm{O}-\beta$-glucoside) were evaluated in a preclinical in vivo study from 2017. The tests were aiming to elucidate the extract's influence on counteracting $\mathrm{CCl}_{4}$-induced liver damage in rats. Although the influence of the extract and compounds on the ALT and AST (aspartate transaminase) levels was insignificant, the histological condition of animal livers was notably better in most samples (except for hydrangenol-8-O$\beta$-glucoside and scorzotomentosin- $4^{\prime}-O-\beta$-glucoside - treated groups). What is interesting in terms of future clinical research is the fact that chlorogenic acid was the most active compound in the treatment of acute carbon tetrachloride-induced liver toxicity [73].

\subsection{Antimicrobial Activity}

Folk medicine has been treating microbial infections for centuries. Along with the decrease in bacteria's susceptibility to antibiotics, the need for novel antimicrobial drugs is increasing. Plants have been a source of folk medications in the treatment of infectious diseases before the concept of infectious agents emerged [88]. The activity against microbes could also be used to substitute synthetic additives in food to prevent foodborne diseases induced by pathogenic bacteria [89] Several species within the genus Scorzonera have been investigated as a source of products with antimicrobial potential.

Volatile oil distilled from aerial parts of Scorzonera undulata ssp. deliciosa was assessed as an antibacterial agent against Gram-positive (Staphylococcus aureus, Staphylococcus epidermidis, and Micrococcus luteus) and Gram-negative (Salmonella typhimurium, Escherichia coli, and Pseudomonas aeruginosa) bacteria strains and it was reported more active towards Grampositive strains with MIC (minimal inhibitory concentration) values of $0.5 \mathrm{mg} / \mathrm{mL}$ for $S$. aureus and M. luteus and $0.8 \mathrm{mg} / \mathrm{mL}$ for S. epidermidis, S. typhimurium, and E. coli. MBC (minimal bactericidal concentration) was not determined for any Gram-negative strain. The authors suggest that greater activity against Gram-positive bacteria strains could be caused by easier penetration through the lipophilic cell membranes by hydrophobic ingredients of the oil. Unfortunately, no reference compound was assessed together with the oil, thus it is difficult to compare those results with any known antibacterial substances [90].

Antibacterial properties of $S$. undulata were assayed in 2010 by Abdelkader and colleagues [91]. The study showed that ethyl acetate fraction of the aerial part methanol extract exhibited antibacterial properties against $P$. aeruginosa, S. aureus, E. faecalis, $C$. freundei, and $P$. mirabilis with MIC exceeding $1 \mathrm{mg} / \mathrm{mL}$. The petroleum ether fraction; however, was active against $P$. aeruginosa, $S$. aureus, and $C$. freundei. Fractions obtained from the roots had a narrower spectrum of activity, but the petroleum ether fraction exhibited a stronger antimicrobial potential against $S$. aureus with a MIC of $500 \mu \mathrm{g} / \mathrm{mL}$.

Bactericidal properties of compounds isolated from a Scorzonera divaricata aerial parts petroleum ether/diethyl ether/methanol extract were evaluated in a study by $\mathrm{Wu}$ and colleagues in 2018 [34]. Sulfoscorzonin D, a new, rare pyrrolidine salt alkaloid obtained in the study, exhibited more potent activity against Clostridium perfingens than ampicillin, its activity was similar to the activity of erythromycin and streptomycin. Sacrolide A was more effective against Newman WT than streptomycin $(12.5$ to $25 \mu \mathrm{g} / \mathrm{mL})$ and similarly effective to levofloxacin $(12.5 \mu \mathrm{g} / \mathrm{mL})$. Other compounds which were reported to possess antibacterial properties (B. megaterum, C. perfingens, Newman WT, and E. coli) were oleanolic acid, lup-20(29)-ene3 $\beta, 28$-diol, (22E)- $5 \alpha, 8 \alpha$-epidioxyergosta-6,22-dien-3 $\beta$-ol, ergosta-3 $\beta, 5 \alpha, 6 \beta$-trialcohol, and diosmetin (MIC values were $12.5-100 \mu \mathrm{g} / \mathrm{mL}$ ).

A comprehensive study on endemic to Lebanon species Scorzonera mackmeliana in terms of the plant's antibacterial and antibiofilm properties was carried out by Sweidan and colleagues in 2020 [92]. The authors conducted a phytoanalysis of the constituents of water and ethanol extracts of the whole plant as well as its particular parts (flowers, 
stems, leaves, roots) and determined their activity against Gram-positive (Staphylococcus epidermidis, Staphylococcus aureus, Enterococcus faecalis) and Gram-negative (Escherichia coli, Pseudomonas aeruginosa) bacterial strains. The highest activities in inhibition of the bacteria strains were observed in two water extracts: the ones obtained from the stems and the whole plant. For the stem water extract, the inhibitory effect was observed in four out of five tested strains, the lowest MIC values were reported in P. aeruginosa $(48.98 \mathrm{mg} / \mathrm{mL})$, S. aureus $(48.98 \mathrm{mg} / \mathrm{mL})$, and S. epidermidis $(48.98 \mathrm{mg} / \mathrm{mL})$. For the water extract from the whole plant, it was proven active against S. epidermidis, P. aeruginosa, and E. coli in the concentration of $122.25 \mathrm{mg} / \mathrm{mL}$ for each strain. MBC was determined for only two extracts and those were root ethanol extract (for S. epidermidis $\mathrm{MBC}=284.35 \mathrm{mg} / \mathrm{mL}$ ) and water flower extract (for P. aeruginosa, $\mathrm{MBC}=160.85 \mathrm{mg} / \mathrm{mL}$ ). Those values; however, are notably high, compared to the literature data available for other plant water and ethanol extracts. MIC and MBC values for Cinnamomum impressicostatum stem bark extract against MRSA strain were 19.53 and $39.06 \mu \mathrm{g} / \mathrm{mL}$, respectively. For Cinnamomum porrectum stem bark extract, it was 2.5 (MIC) and $5 \mathrm{mg} / \mathrm{mL}$ (MBC) [93]. In the Asteraceae family, water extracts from Sonchus erzincanicus aerial parts exhibit antibacterial properties against Staphylococcus aureus, Escherichia coli, and Proteus mirabilis with the MIC value of $1.25 \mathrm{mg} / \mathrm{mL}$ [94]. In the mentioned study on S. mackmeliana; however, an interesting pattern of the activity against biofilm formation was observed-with the concentration decrease, greater destruction of the biofilm occurred. It was also observed that flower and stem water extracts and ethanol leaf, flower, and whole plant extracts exhibited the most potent activity in eradicating bacterial biofilm, with MBEC (minimal biofilm eradication concentration) values of $0.1-2.2 \mathrm{mg} / \mathrm{mL}$, causing $84-98 \%$ biofilm eradication. For water extracts, the presence of coumarin was suspected to be the active factor and in ethanol extracts, terpenoids were major constituents and; therefore, they are thought to be responsible for the antibiofilm effect [92].

The antibacterial and antifungal activity of the aerial parts and root extracts from $S$. papposa was investigated in a recent study by Mohammed and colleagues [95]. The extracts were toxic for bacteria at the concentrations of $50-800 \mu \mathrm{g} / \mathrm{mL}$ and their antifungal effectiveness was observed at 50-100 $\mu \mathrm{g} / \mathrm{mL}$. The reference compounds (ampicillin, amikacin, ciprofloxacin, fluconazole, and amphotericin B) on the other hand were active at notably lower concentrations (1.56-3.12 $\mu \mathrm{g} / \mathrm{mL})$. In general, samples collected in Turkey were slightly more effective against both bacteria and fungi $(50 \mu \mathrm{g} / \mathrm{mL}$ against Pseudomonas aeruginosa), although all tested extracts can potentially be used as mild nature-derived antimicrobial agents.

In a study on antimicrobial activities of compounds isolated from aerial parts of Scorzonera aucheriana it was observed that scorzoaucherioside II, iso-scorzopygmaecoside, and 3,4-dihydroxyphenyl caffeate possess strong anti-tuberculosis activity with MIC of $21.2 \mu \mathrm{g} / \mathrm{mL}, 25.6 \mu \mathrm{g} / \mathrm{mL}$, and $145 \mu \mathrm{g} / \mathrm{mL}$, respectively. 3,4-Dihydroxyphenyl caffeate and scorzoaucherioside I were reported active against Gram-negative bacteria strain Pseudomonas aeruginosa (MIC $=290$ and $377.5 \mu \mathrm{g} / \mathrm{mL}$, respectively). Enterococcus faecalis, a Gram-positive strain, was reported sensitive to scorzopygmaecoside and scorzocreticoside II, where MIC values were 135 and $200 \mu \mathrm{g} / \mathrm{mL}$, respectively [7].

\subsection{Wound Healing Activity}

Wounds defined as a disruption of tissue do not pose a threat unless the blood loss is significant. They can; however, be a gateway for pathogenic infections which is much more dangerous for the patient. The acceleration of wound recovery includes infection prevention and the promotion of the natural healing process. Medicinal plants are suspected to possess both those qualities and are considered potentially effective in the therapy of wound healing [96].

\subsubsection{In Vitro Assays}

A study carried out by Küpeli Akkol and colleagues in 2019 [4] reports that ethyl acetate and chloroform fractions of a methanol extract from aerial parts of S. latifolia have 
wound healing properties in vitro, which are a result of the inhibitory effect on collagenase and elastase enzymes activity. None of the fractions influenced the activity of hyaluronidase to any considerable degree.

In a study on the extract from S. cana var. jacquiniana aerial parts (stems, leaves, and flowers), their activity against matrix metalloproteinases (collagenase, hyaluronidase, and elastase) was evaluated. A methanol extract was partitioned into chloroform, petroleum ether, ethyl acetate, and water fraction. From the ethyl acetate fraction, eleven compounds were isolated (3,5-dicaffeoylquinic acid methyl ester, 4-hydroxy-benzoic acid 4-(6-O- $\alpha$-rhamnopyranosyl- $\beta$-glucopyranosyl) benzyl ester, $6^{\prime}$-O-caffeoylarbutin, apigenin 7-O- $\beta$-glucoside, apigenin 7-O- $\beta$-rutinoside, arbutin, cichoriin, isoorientin, luteolin 7-O$\beta$-glucoside, orientin, protocatechuic acid, and vitexin) and all obtained samples were tested for their enzyme-inhibitory properties. In the hyaluronidase inhibition assay, the extracts, fractions, and isolated compounds exhibited only a mild inhibitory effect at the concentration of $100 \mu \mathrm{g} / \mathrm{mL}$ (not exceeding $30 \%$ for luteolin $7-O-\beta$-glucoside), compared to the positive control-Tannic acid-Which inhibited the hyaluronidase activity by approximately $75 \%$. The methanol extract was observed to possess a potent inhibitory effect on both elastase and collagenase activity $(51.7 \%$ inhibition for elastase and $35.7 \%$ for collagenase, in the concentration of $100 \mu \mathrm{g} / \mathrm{mL}$ ). Moreover, several compounds isolated from the extract were significantly active against collagenase (apigenin 7-O- $\beta$-glucoside, apigenin 7-O- $\beta$-rutinoside, and isoorientin) and elastase (apigenin 7-O- $\beta$-glucoside, luteolin 7-O- $\beta$-glucoside, and apigenin 7-O- $\beta$-rutinoside) as well. In light of those results, the authors suggested that flavonoids present in methanol extract were the main woundhealing agents and that the synergy between components of the extract contributes to the inhibitory effect [72].

\subsubsection{In Vivo Assays}

In 2011, a study by Küpeli Akkol et al. [5] on the promotion of the process of wound healing in mice took place. Researchers obtained hydroethanolic extracts from the aerial parts and roots of several Scorzonera species (S. cinerea, S. latifolia, S. incisa, S. mobilis, S. mollis ssp. szowitsii, S. tomentosa). Most promising results in the assays carried out with wound models were observed with ointments made with the extracts obtained from S. latifolia, S. mollis ssp. Szowitsii, and S. tomentosa aerial parts. Those three extracts were the most active in the hydroxyproline level enhancement as wells as in terms of skin remodeling. Moreover, the S. latifolia aerial part extracts were reported to have anti-inflammatory properties in vivo, with an inhibitory value of $23.5 \%$ at the dose of $100 \mathrm{mg} / \mathrm{kg}$.

A study from 2012, carried out on mice, reports wound healing properties of several Scorzonera species. Aqueous methanolic extracts from the aerial parts of $S$. cana var. jacquiniana, S. eriophora, and S. acuminata caused the contraction of wound area by up to $46.27 \%$ on day 12 in the circular excision wound model. Ointments containing extracts of S. cana (C.A. Mey.) Hoffm. var. jacquiniana (W. Koch) Chamb. and S. eriophora DC. aerial parts, when applied topically on the linear incision wound models, caused an increase in the activity of anti-hyaluronidase and significant enhancement of hydroxyproline level in the regenerated tissue [9].

Figure 2 summarizes literature data regarding the biological activity of Scorzonera species in vivo.

\subsection{Antioxidant Capacity}

Exposure to reactive species in the environment may have a negative impact on humans and animals. The balance between oxidants and antioxidants is becoming more difficult to maintain [97].; therefore, the need for antioxidant agents is growing and, because of the potentially harmful effect of synthetic antioxidants, the attention seems to be currently directed towards naturally occurring antioxidants found in plants [98]. The products from species belonging to the genus Scorzonera have been assayed as antioxidant agents as well. 


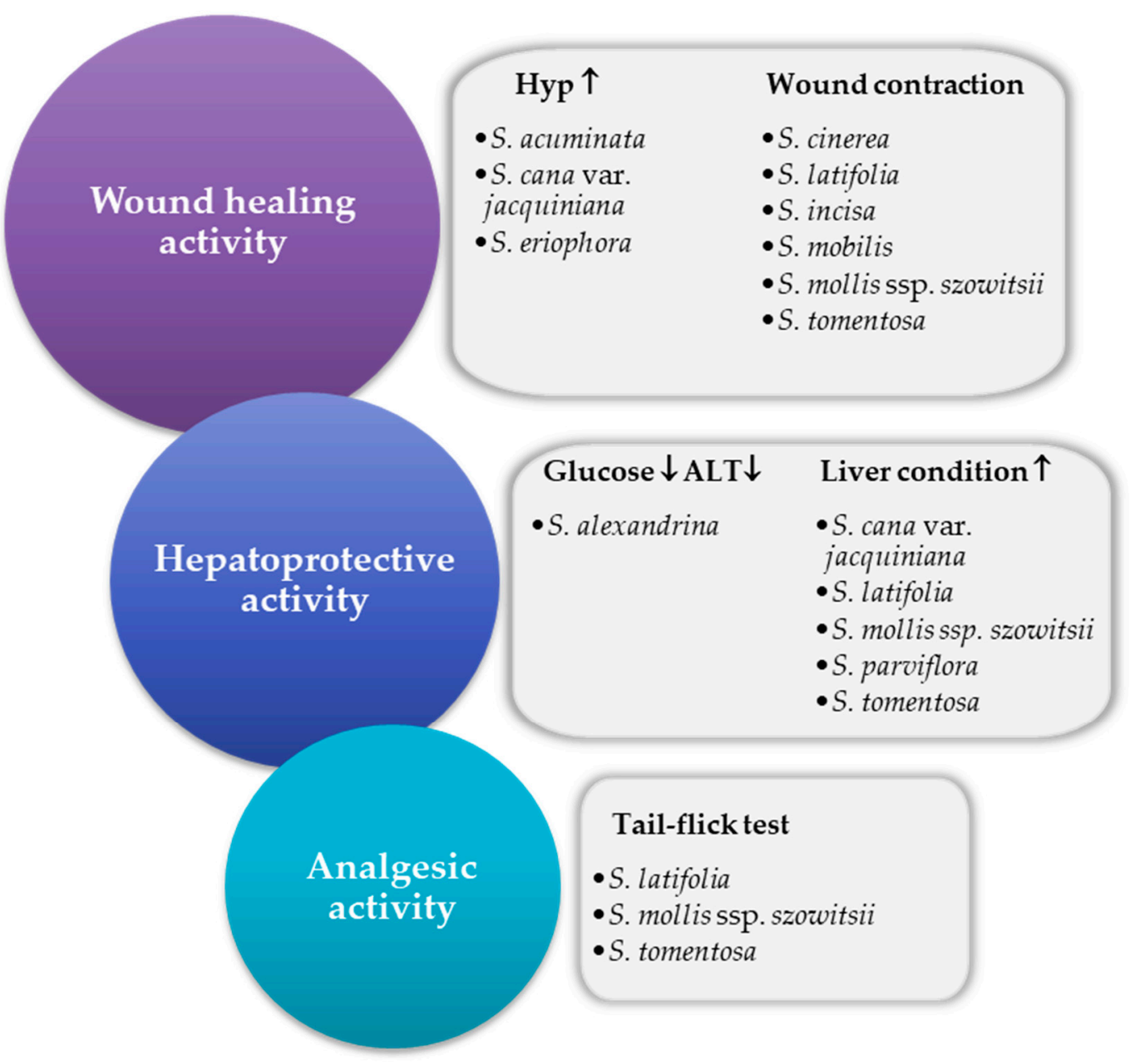

Figure 2. Biological activity of species within the genus Scorzonera evaluated in vivo. Hyp $\uparrow:$ Increase in the hydroxyproline level; Glucose $\downarrow$ : Decrease in the glucose level; ALT $\downarrow$ : Decrease in the alanine transaminase level; Liver condition $\uparrow:$ Improvement in the liver condition.

In the DPPH radical scavenging assay compounds isolated from the ethyl acetate fraction of the methanol extract from Scorzonera divaricata and Scorzonera pseudodivaricata aerial parts (ferulopodospermic acid A and B) did exhibit a strong antioxidant activity, more potent than chlorogenic acid used as a reference compound in the study. The $\mathrm{IC}_{50}$ values ferulopodospermic acid A and B were 36.36 and $34.24 \mu \mathrm{mol} / \mathrm{mL}$, respectively, compared to the $\mathrm{IC}_{50}$ of chlorogenic acid, which was $67.92 \mu \mathrm{mol} / \mathrm{mL}$ [14]. The study was continued with five compounds obtained from a Scorzonera radiata aerial part $\mathrm{MeOH}$ extract were tested for their radical-scavenging activity in the DPPH assay. Scorzodihydrostilbenes A and E exhibited a higher activity level than resveratrol, well-known for its antioxidant activity, used as a reference in the study. The $\mathrm{IC}_{50}$ values were $105.51 \mu \mathrm{M}$ for scorzodihydrostilbene A, $102.60 \mu \mathrm{M}$ for scorzodihydrostilbene B, and $149.52 \mu \mathrm{M}$ for resveratrol [15]. Although the difference in the scale of $\mathrm{IC}_{50}$ values might seem interesting, as $\mathrm{IC}_{50}$ in $S$. divaricata and S. pseudodivaricata was given in $\mu \mathrm{mol} / \mathrm{mL}$, whereas for $S$. radiata it was presented in $\mu \mathrm{M}$ $(\mu \mathrm{mol} / \mathrm{L})$, more informative is how those results correspond to reference compounds used in both studies.

In the assessment of antioxidant activity, acteoside isolated from a methanolic extract from Scorzonera undulata ssp. deliciosa roots was reported to possess similar antiradical power to Trolox used as a standard in the DPPH test $\left(\mathrm{IC}_{50}\right.$ values were $0.16 \pm 0.02 \mathrm{mg} / \mathrm{mg}$ DPPH for acteoside and $0.2 \pm 0.01 \mathrm{mg} / \mathrm{mg}$ DPPH for Trolox). The Trolox Equivalent Antioxidant Capacity (TEAC) value of acteoside in the DPPH assay was 1.25. In the CUPRAC (cupric 
reducing antioxidant capacity) assay acteoside was slightly less active than the reference compound, rutin (TEAC $=3.16$ for rutin compared to TEAC $=2.4$ for acteoside) [49].

Nasseri et al. [52] evaluated the chemical composition and the radical scavenging activity of Scorzonera paradoxa root and leaf ethanol/water extracts. Leaf extracts turned out to be a more potent antioxidant with an $\mathrm{IC}_{50}$ value of $18.81 \mathrm{mg} / \mathrm{mL}$, compared to the roots $\left(\mathrm{IC}_{50}=88.9 \mathrm{mg} / \mathrm{mL}\right)$. This may be due to higher levels of phenolic compounds, flavonoids and tannins reported in the study. The authors also made an assessment of the fatty acids composition of the plant samples and based on the data obtained in the study of chemical composition and antioxidant properties, it was suggested that $S$. paradoxa might be successful as an antidiabetic agent. Those results and IC $_{50}$ values correspond with a study from 2013, when Erden and colleagues [22] investigated the antioxidant properties of methanol extracts obtained from the leaves of three Scorzonera species (S. suberosa, $S$. laciniata, and S. latifolia). Those properties were examined in the DPPH assay and exhibited a concentration-dependent antioxidant activity with $\mathrm{IC}_{50}$ values of $29.36 \mathrm{mg} / \mathrm{mL}$ for $S$. latifolia, $42.33 \mathrm{mg} / \mathrm{mL}$ for S. suberosa, and $77.07 \mathrm{mg} / \mathrm{mL}$ for S. laciniata.

In 2013 Milella et al. [62] measured the antioxidant properties of pure compounds isolated from methanol extracts from aerial parts and roots of Scorzonera papposa. The authors assessed the antioxidant activity of the compounds obtained from S. judaica in Bader's previous study from 2011 [25] as well. Four out of nine compounds isolated from S. papposa extracts were previously unknown. In the study, the antioxidant activity was measured in four different assays: the DPPH assay, the FRAP (ferric reducing antioxidant power) assay, the BCB ( $\beta$-Carotene bleaching) assay, and the TPC (total phenolic content) assay. It has been observed that the antioxidant capacity of particular compounds depends on the method. The authors suggested that the antioxidant activity of the compounds found in the extract is a result of the synergistic effect of their combination. In the study, a new concept for presenting the antioxidant capacity of compounds-Relative Antioxidant Capacity Index (RACI) - Was applied [62]. Briefly, the parameter is used to integrate the data from several methods for the assessment of the antioxidant activity, where each method is assigned equal weight. RACI can take positive or negative values [99].

Yang and colleagues [59] assessed the antioxidant properties of several compounds isolated from an ethanol Scorzonera divaricata root extract. In the ABTS antioxidant capacity assay, two compounds obtained in the study- $(1 R, 5 S, 6 S, 7 R, 8 S)$-8-sulfoxyguaia4(15),10(14),11(13)-trine-6,12-olide (sulfoscorzonin A) and (1R,5S,6S,7R,8S,13S)-8-sulfoxy13-L-prolineyl-guaia-4(15),10(14)-dien-6a,12-olide (sulfoscorzonin C)-Were reported to be moderately active in radical scavenging. The $\mathrm{SC}_{50}$ (half-maximal scavenging concentration) values were equal to $32.88 \mu \mathrm{M}$ for sulfoscorzonin A and $24.86 \mu \mathrm{M}$ for sulfoscorzonin C.

A recent study on Scorzonera papposa was a comparison of the antioxidant and antimicrobial activity of ethanol extracts from aerial parts and roots of S. papposa from Iraq and Turkey. It has been observed that samples from Iraq exhibited a higher level of TAS (Total Antioxidant Status) and a lower level of TOS (Total Oxidant Status) than samples collected in Turkey. Therefore, the OSI (Oxidative Stress Index-The TAS/TOS ratio) parameter in the samples from Iraq was lower than in the samples from Turkey. Compared to the reported TAS and TOS values of other plant species (i.e., Calendula officinalis L., Rhus coriaria L. var. zebaria, Shahbaz and Mentha longifolia L.), the extracts from the aerial parts of $S$. papposa obtained in the study exhibited a notable antioxidant activity [95].

\section{Conclusions}

Aerial and subaerial parts of species within the Scorzonera genus have been the subject of research regarding their phytochemical composition as well as their therapeutic potential. In many European and Asian cultures, Scorzonera species are commonly used in folk medicine,; therefore, modern phytoanalyses and biological studies have been carried out to verify the bioactive activities of the plants. Due to the presence of numerous bioactive compounds, including flavonoid aglycones and glycosides, triterpenoids, sesquiterpenoids, quinic acid, and caffeic acid derivatives, in the studied plant material, Scorzonera species 
are considered a potential source of antioxidant agents. Although the reported cytotoxicity of Scorzonera extract against cancer cell lines so far was insignificant, they exhibit other bioactive properties, potentially applicable not only in the therapy of pain, inflammation, and microbial infections, but also as an enhancement of the effectiveness of the wound healing process. It should be pointed out that a promising direction of further research on the genus Scorzonera is the investigation of their activity towards normal cell lines, especially skin cells, to assess their potential as wound-healing and skincare active agents.

Funding: This research was funded by Medical University of Bialystok, grant numberSUB/2/DN/ 21/002/2229 (approval date: 4 January 2021).

Institutional Review Board Statement: Not applicable.

Informed Consent Statement: Not applicable.

Data Availability Statement: Not applicable.

Conflicts of Interest: The authors declare no conflict of interest.

\section{Abbreviations}

\begin{tabular}{|c|c|}
\hline ALT & alanine transaminase \\
\hline AST & aspartate transaminase \\
\hline $\mathrm{BCB}$ & beta-carotene bleaching \\
\hline $\mathrm{CCl}_{4}$ & carbon tetrachloride \\
\hline $\mathrm{CHCl}_{3}$ & chloroform \\
\hline COX-1 & cyclooxygenase 1 \\
\hline COX-2 & cyclooxygenase 2 \\
\hline CUPRAC & cupric reducing antioxidant capacity \\
\hline FRAP & ferric reducing antioxidant activity \\
\hline $\mathrm{H}_{2} \mathrm{O}_{2}$ & hydrogen peroxide \\
\hline $\mathrm{H}_{2} \mathrm{SO}_{4}$ & sulfuric acid \\
\hline $\mathrm{HNO}_{3}$ & nitric acid \\
\hline Hyp & hydroxyproline \\
\hline $\mathrm{IC}_{50}$ & half-maximal inhibitory concentration \\
\hline IL-1 $\beta$ & interleukin $1 \beta$ \\
\hline $\mathrm{MBC}$ & minimal bactericidal concentration \\
\hline MIC & minimal inhibitory concentration \\
\hline$N F-\kappa B$ & nuclear factor kappa B \\
\hline OSI & oxidative stress index \\
\hline RACI & relative antioxidant capacity index \\
\hline $\mathrm{SC}_{50}$ & half-maximal scavenging concentration \\
\hline TAS & total antioxidant status \\
\hline TEAC & Trolox equivalent antioxidant capacity \\
\hline TNF- $\alpha$ & tumor necrosis factor $\alpha$ \\
\hline TOS & total oxidant status \\
\hline TPC & total phenolic content \\
\hline
\end{tabular}

\section{References}

1. Duran, A.; Hamzaoğlu, E. A new species of Scorzonera L. (Asteraceae) from South Anatolia, Turkey. Biologia 2004, 59, 47-50.

2. Karaer, F.; Celep, F. Rediscovery of Scorzonera amasiana Hausskn. and Bornm.-A threatened endemic species in Turkey. Bangladesh J. Bot. 2007, 36, 139-144. [CrossRef]

3. Bahadir-Acikara, Ö.; Citoğlu-Gülçin, S.; Dall'Acqua, S.; Özbek, H.; Cvačka, J.; Žemlička, M.; Šmejkal, K. Bioassay-guided isolation of the antinociceptive compounds motiol and $\beta$-sitosterol from Scorzonera latifolia root extract. Pharmazie 2014, 69, 711-714. [CrossRef]

4. Küpeli-Akkol, E.; Šmejkal, K.; Kurtul, E.; Ilhan, M.; Güragac, F.T.; Çitoğlu, G.S.; Acıkara, Ö.B.; Cvačka, J.; Buděšínský, M. Inhibitory activity of Scorzonera latifolia and its components on enzymes connected with healing process. J. Ethnopharmacol. 2019, 245. [CrossRef] 
5. Küpeli-Akkol, E.; Acikara, O.B.; Süntar, I.; Citolu, G.S.; Kele, H.; Ergene, B. Enhancement of wound healing by topical application of Scorzonera species: Determination of the constituents by HPLC with new validated reverse phase method. J. Ethnopharmacol. 2011, 137, 1018-1027. [CrossRef]

6. Bahadır, Ö.; Citoğlu, G.S.; Smejkal, K.; Dall'Acqua, S.; Ozbek, H.; Cvacka, J.; Zemlicka, M. Analgesic compounds from Scorzonera latifolia (Fisch. and Mey.) DC. J. Ethnopharmacol. 2010, 131, 83-87. [CrossRef] [PubMed]

7. Erik, İ.; Yaylı, N.; Coşkunçelebi, K.; Makbul, S.; Karaoğlu, Ş.A. Three new dihydroisocoumarin glycosides with antimicrobial activities from Scorzonera aucheriana. Phytochem. Lett. 2021, 43, 45-52. [CrossRef]

8. Sarı, A.; Şahin, H.; Özsoy, N.; Özbek Çelik, B. Phenolic compounds and in vitro antioxidant, anti-inflammatory, antimicrobial activities of Scorzonera hieraciifolia Hayek roots. S. Afr. J. Bot. 2019, 125, 116-119. [CrossRef]

9. Süntar, I.; Bahadır-Acıkara, Ö.; Saltan-Çitoğlu, G.; Keleş, H.; Ergene, B.; Küpeli Akkol, E. In vivo and in vitro evaluation of the therapeutic potential of some Turkish Scorzonera species as wound healing agent. Curr. Pharm. Des. 2012, 18, 1421-1433. [CrossRef]

10. Bahadır-Acikara, Ö.; Hošek, J.; Babula, P.; Cvačka, J.; Budešínský, M.; Dračinský, M.; Saltan İşcan, G.; Kadlecová, D.; Ballová, L.; Šmejkal, K. Turkish Scorzonera species Extracts attenuate cytokine secretion via inhibition of NF-kB activation, showing anti-inflammatory effect in vitro. Molecules 2016, 21, 43. [CrossRef]

11. Coşkunçelebi, K.; Makbul, S.; Gültepe, M.; Okur, S.; Güzel, M.E. A conspectus of Scorzonera s.l. in Turkey. Turk. J. Bot. 2015, 39, 76-87. [CrossRef]

12. Tsevegsuren, N.; Proksch, P.; Wang, Y.; Davaakhuu, G. Bioactive phenolic acids from Scorzonera radiata Fisch. Mong. J. Chem. 2014, 12, 78-84. [CrossRef]

13. Wang, Y.; Wray, V.; Tsevegsuren, N.; Lin, W.; Proksch, P. Phenolic compounds from the Mongolian medicinal plant Scorzonera radiata. Z. Naturforsch. Sect. C J. Biosci. 2012, 67, 135-143. [CrossRef]

14. Tsevegsuren, N.; Edrada, R.A.; Lin, W.; Ebel, R.; Torre, C.; Ortlepp, S.; Wray, V.; Proksch, P. Biologically active natural products from Mongolian medicinal plants Scorzonera divaricata and Scorzonera pseudodivaricata. J. Nat. Prod. 2007, 70, 962-967. [CrossRef]

15. Wang, Y.; Edrada-Ebel, R.; Tsevegsuren, N.; Sendker, J.; Braun, M.; Wray, V.; Lin, W.; Proksch, P. Dihydrostilbene derivatives from the mongolian medicinal plant Scorzonera radiata. J. Nat. Prod. 2009, 72, 671-675. [CrossRef]

16. Li, J.; Wu, Q.X.; Shi, Y.P.; Zhu, Y. A new sesquiterpene lactone from Scorzonera austriaca. Chin. Chem. Lett. 2004, 15, 1309-1310.

17. Zhu, Y.; Wu, Q.X.; Hu, P.Z.; Wu, W.S. Biguaiascorzolides A and B: Two novel dimeric guaianolides with a rare skeleton, from Scorzonera austriaca. Food Chem. 2009, 114, 1316-1320. [CrossRef]

18. Wang, B.; Li, G.Q.; Qiu, P.J.; Guan, H.S. Two new olean-type triterpene fatty esters from Scorzonera mongolica. Chin. Chem. Lett. 2007, 18, 708-710. [CrossRef]

19. Zaika, M.A.; Kilian, N.; Jones, K.; Krinitsina, A.A.; Nilova, M.V.; Speranskaya, A.S.; Sukhorukov, A.P. Scorzonera sensu lato (Asteraceae, Cichorieae)-Taxonomic reassessment in the light of new molecular phylogenetic and carpological analyses. PhytoKeys 2020, 137, 1-85. [CrossRef]

20. Granica, S.; Lohwasser, U.; Jöhrer, K.; Zidorn, C. Qualitative and quantitative analyses of secondary metabolites in aerial and subaerial of Scorzonera hispanica L. (black salsify). Food Chem. 2015, 173, 321-331. [CrossRef]

21. Buranov, A.U.; Elmuradov, B.J. Extraction and characterization of latex and natural rubber from rubber-bearing plants. J. Agric. Food Chem. 2010, 58, 734-743. [CrossRef] [PubMed]

22. Erden, Y.; Kırbağ, S.; Yılmaz, Ö. Phytochemical composition and antioxidant activity of some Scorzonera species. Proc. Natl. Acad. Sci. India Sect. B Biol. Sci. 2013, 83, 271-276. [CrossRef]

23. Benabdelaziz, I.; Haba, H.; Lavaud, C.; Benkhaled, M. Triterpenoids and flavonoid from Scorzonera undulata ssp. alexandrina. Int. J. Chem. Biochem. Sci. 2014, 5, 1-5.

24. Xie, Y.; Guo, Q.S.; Wang, G.S. Flavonoid glycosides and their derivatives from the herbs of Scorzonera austriaca Willd. Molecules 2016, 21, 803. [CrossRef]

25. Bader, A.; de Tommasi, N.; Cotugno, R.; Braca, A. Phenolic compounds from the roots of jordanian viper's grass, Scorzonera judaica. J. Nat. Prod. 2011, 74, 1421-1426. [CrossRef]

26. Granica, S.; Zidorn, C. Phenolic compounds from aerial parts as chemosystematic markers in the Scorzonerinae (Asteraceae). Biochem. Syst. Ecol. 2015, 58, 102-113. [CrossRef]

27. Bahadır-Acıkara, Ö.; Özbilgin, S.; Saltan-İşcan, G.; Dall’Acqua, S.; Rjašková, V.; Özgökçe, F.; Suchý, V.; Šmejkal, K. Phytochemical analysis of Podospermum and Scorzonera $n$-hexane extracts and the HPLC quantitation of triterpenes. Molecules 2018, 23, 1813. [CrossRef]

28. Wu, Q.X.; Su, Y.B.; Zhu, Y. Triterpenes and steroids from the roots of Scorzonera austriaca. Fitoterapia 2011, 82, 493-496. [CrossRef] [PubMed]

29. Erik, I.; Coşkunçelebi, K.; Makbul, S.; Yayli, N. New chlorogenic acid derivatives and triterpenoids from Scorzonera aucheriana. Turk. J. Chem. 2021, 45, 199-209. [CrossRef]

30. Çetin, B.; Şahin, H.; Sarı, A. Triterpenoids from Scorzonera veratrifolia Fenzl. Istanbul J. Pharm. 2019, 48, 23-27. [CrossRef]

31. Wang, B.; Li, G.-Q.; Guan, H.; Yang, L.; Tong, G. A new erythrodiol triterpene fatty ester from Scorzonera mongolica. Yao Xue Xue Bao 2009, 44, 1258-1261. [PubMed]

32. Zidorn, C.; Ellmerer-Müller, E.P.; Stuppner, H. Sesquiterpenoids from Scorzonera hispanica L. Pharmazie 2000, 55, 550-551. [PubMed] 
33. Zhu, Y.; Hu, P.Z.; He, Z.W.; Wu, Q.X.; Li, J.; Wu, W.S. Sesquiterpene lactones from Scorzonera austriaca. J. Nat. Prod. 2010, 73, 237-241. [CrossRef]

34. Wu, Q.X.; He, X.F.; Jiang, C.X.; Zhang, W.; Shi, Z.N.; Li, H.F.; Zhu, Y. Two novel bioactive sulfated guaiane sesquiterpenoid salt alkaloids from the aerial parts of Scorzonera divaricata. Fitoterapia 2017, 124, 113-119. [CrossRef] [PubMed]

35. Şahin, H.; Sarı, A.; Özsoy, N.; Özbek Çelik, B.; Koyuncu, O. Two new phenolic compounds and some biological activities of Scorzonera pygmaea Sibth. and Sm. subaerial parts. Nat. Prod. Res. 2020, 34, 621-628. [CrossRef] [PubMed]

36. Sarı, A.; Zidorn, C.; Ellmerer, E.P.; Özgökçe, F.; Ongania, K.H.; Stuppner, H. Phenolic compounds from Scorzonera tomentosa L. Helv. Chim. Acta 2007, 90, 311-317. [CrossRef]

37. Paraschos, S.; Magiatis, P.; Kalpoutzakis, E.; Harvala, C.; Skaltsounis, A.L. Three new dihydroisocoumarins from the Greek endemic species Scorzonera cretica. J. Nat. Prod. 2001, 64, 1585-1587. [CrossRef]

38. Karakaya, S.; Polat, A.; Aksakal, Ö.; Sümbüllü, Y.Z.; İncekara, Ü. Ethnobotanical study of medicinal plants in aziziye district (Erzurum, Turkey). Turk. J. Pharm. Sci. 2020, 17, 211-220. [CrossRef]

39. Yaldiz, G.; Koca Çalişkan, U.; Aka, C. In vitro screening of natural drug potentials for mass production. Not. Bot. Horti Agrobot. Cluj-Napoca 2017, 45, 292-300. [CrossRef]

40. De Smet, P.A.G.M. The Role of Plant-Derived Drugs and Herbal Medicines in Healthcare. Drugs 1997, 54, 801-840. [CrossRef]

41. Saklani, A.; Kutty, S.K. Plant-derived compounds in clinical trials. Drug Discov. Today 2008, 13. [CrossRef] [PubMed]

42. Li, F.S.; Weng, J.K. Demystifying traditional herbal medicine with modern approaches. Nat. Plants 2017, 3, 1-7. [CrossRef] [PubMed]

43. Patridge, E.; Gareiss, P.; Kinch, M.S.; Hoyer, D. An analysis of FDA-approved drugs: Natural products and their derivatives. Drug Discov. Today 2016, 21, 204-207. [CrossRef] [PubMed]

44. Rees, S.; Harborne, J. Flavonoids and other phenolics of Cichorium and related members of the Lactuceae (Compositae). Bot. J. Linn. Soc. 1984, 89, 313-319. [CrossRef]

45. Bryanskii, O.V.; Tolstikhina, V.V.; Zinchenko, S.V.; Semenov, A.A. A sesquiterpene glucoside from cultivated cells of Scorzonera hispanica. Chem. Nat. Compd. 1992, 28, 556-560. [CrossRef]

46. Tolstikhina, V.V.; Bryanskii, O.V.; Syrchina, A.I.; Semenov, A.A. Chemical composition of a culture of tissue of Scorzonera hispanica. Chem. Nat. Compd. 1988, 24, 655. [CrossRef]

47. Bahadır-Acıkara, Ö.; Saltan-Çitoğlu, G.; Dall'Acqua, S.; Šmejkal, K.; Cvačka, J.; Žemlička, M. A new triterpene from Scorzonera latifolia (Fisch. and Mey.) DC. Nat. Prod. Res. 2012, 26, 1892-1897. [CrossRef]

48. Yildirim, B.; Terzioglu, Ö.; Özgökçe, F.; Türközü, D. Ethnobotanical and pharmacological uses of some plants in the districts of Karpuzalan and Adigüzel (Van-Turkey). J. Anim. Vet. Adv. 2008, 7, 873-878.

49. Harkati, B.; Salah, A.; Bayet, C.; Laouer, H.; Dijoux-Franca, M.-G. Evaluation of antioxidant activity, free radical scavenging and CUPRAC of two compounds isolated from Scorzonera undulata ssp. deliciosa. Adv. Environ. Biol. 2013, 7, 591-594.

50. Sharma, J.; Gairola, S.; Gaur, R.D.; Painuli, R.M. The treatment of jaundice with medicinal plants in indigenous communities of the Sub-Himalayan region of Uttarakhand, India. J. Ethnopharmacol. 2012, 143, 262-291. [CrossRef]

51. Auzi, A.R.A.; Hawisa, N.T.; Sherif, F.M.; Sarker, S.D. Neuropharmacological properties of Launaea resedifolia. Rev. Bras. Farmacogn. 2007, 17, 160-165. [CrossRef]

52. Nasseri, M.A.; Bigy, S.S.; Allahresani, A.; Malekaneh, M. Assessment of antioxidant activity, chemical characterization and evaluation of fatty acid compositions of Scorzonera paradoxa Fisch and C. A. Mey. Jundishapur J. Nat. Pharm. Prod. 2015, 10. [CrossRef]

53. Zidorn, C.; Spitaler, R.; Ellmerer-Müller, E.P.; Perry, N.B.; Gerhäuser, C.; Stuppner, H. Structure of tyrolobibenzyl D and biological activity of tyrolobibenzyls from Scorzonera humilis. Z. Naturforsch. Sect. C J. Biosci. 2002, 57, 614-619. [CrossRef]

54. Zidorn, C.; Ellmerer-Müller, E.P.; Stuppner, H. Tyrolobibenzyls-Novel secondary metabolites from Scorzonera humilis. Helv. Chim. Acta 2000, 83, 2920-2925. [CrossRef]

55. Zidorn, C.; Ellmerer, E.P.; Sturm, S.; Stuppner, H. Tyrolobibenzyls E and F from Scorzonera humilis and distribution of caffeic acid derivatives, lignans and tyrolobibenzyls in European taxa of the subtribe Scorzonerinae (Lactuceae, Asteraceae). Phytochemistry 2003, 63, 61-67. [CrossRef]

56. Jehle, M.; Bano, J.; Ellmerer, E.; Zidorn, C. Natural products from Scorzonera aristata (Asteraceae). Nat. Prod. Commun. 2010, 5, 725-727. [CrossRef]

57. Meng, X.H.; Yang, Y.J.; Gong, Y.; Zhu, Y. Chemical constituents of the roots of Scorzonera divaricata and their chemotaxonomic significance. Biochem. Syst. Ecol. 2020, 93, 104135. [CrossRef]

58. Yang, Y.-J.; Liu, X.; Wu, H.-R.; He, X.-F.; Bi, Y.-R.; Zhu, Y.; Liu, Z.-L. Radical scavenging activity and cytotoxicity of active quinic acid derivatives from Scorzonera divaricata roots. Food Chem. 2013, 138, 2057-2063. [CrossRef]

59. Yang, Y.-J.; Yao, J.; Jin, X.-J.; Shi, Z.-N.; Shen, T.-F.; Fang, J.-G.; Yao, X.-J.; Zhu, Y. Sesquiterpenoids and tirucallane triterpenoids from the roots of Scorzonera divaricata. Phytochemistry 2016, 124, 86-98. [CrossRef] [PubMed]

60. Petkova, N. Characterization of inulin from black salsify (Scorzonera hispanica L.) for food and pharmaceutical purposes. Asian J. Pharm. Clin. Res. 2018, 11, 221-225. [CrossRef]

61. Zidorn, C.; Gottschlich, G.; Stuppner, H. Chemosystematic investigations on phenolics from flowerheads of Central European taxa of Hieracium sensu lato (Asteraceae). Plant Syst. Evol. 2002, 231, 39-58. [CrossRef] 
62. Milella, L.; Bader, A.; de Tommasi, N.; Russo, D.; Braca, A. Antioxidant and free radical-scavenging activity of constituents from two Scorzonera species. Food Chem. 2014, 160, 298-304. [CrossRef] [PubMed]

63. Harkati, B.; Akkal, S.; Bayat, C.; Laouer, H.; Dijoux Franca, M.G. Secondary metabolites from Scorzonera undulata ssp. deliciosa (Guss.) Maire (Asteraceae) and their antioxidant activities. Rec. Nat. Prod. 2010, 4, 171-175.

64. Sar1, A. Two new 3-benzylphthalides from Scorzonera veratrifolia Fenzl. Nat. Prod. Res. 2010, 24, 56-62. [CrossRef]

65. Scarano, A.; Chieppa, M.; Santino, A. Looking at flavonoid biodiversity in horticultural crops: A colored mine with nutritional benefits. Plants 2018, 7, 98. [CrossRef]

66. Agati, G.; Azzarello, E.; Pollastri, S.; Tattini, M. Flavonoids as antioxidants in plants: Location and functional significance. Plant Sci. 2012, 196, 67-76. [CrossRef]

67. Sar1, A. Phenolic compounds from Scorzonera latifolia (Fisch. and Mey.) DC. Nat. Prod. Res. 2012, 26, 50-55. [CrossRef]

68. Bahadır-Acıkara, Ö.; Küpeli-Akkol, E.; Süntar, I.; Ergene, B.; Saltan-Çitoğlu, G.; Çoban, T. Assessment of anti-inflammatory and free radical scavenger activities of selected Scorzonera species and determination of active components. Int. J. Pharmacogn. Phytochem. Res. 2014, 6, 492-498.

69. Turan, M.; Kordali, S.; Zengin, H.; Dursun, A.; Sezen, Y. Macro and micro mineral content of some wild edible leaves consumed in Eastern Anatolia. Acta Agric. Scand. Sect. B Soil Plant Sci. 2003, 53, 129-137. [CrossRef]

70. Bahadır, Ö.; Saltan, H.G.; Özbek, H. Antinociceptive activity of some Scorzonera L. species. Turk. J. Med. Sci. 2012, 42, 861-866. [CrossRef]

71. Bahadır-Acıkara, Ö.; Smejkal, K.; Cvačka, J.; Buděšínský, M.; Dračínský, M.; Saltan, G. Secondary metabolites from Scorzonera latifolia roots. Planta Med. 2015, 81. [CrossRef]

72. Bahadır-Acıkara, Ö.; Ilhan, M.; Kurtul, E.; Šmejkal, K.; Küpeli Akkol, E. Inhibitory activity of Podospermum canum and its active components on collagenase, elastase and hyaluronidase enzymes. Bioorg. Chem. 2019, 93. [CrossRef] [PubMed]

73. Özbek, H.; Bahadir, O.; Keskin, I.; Kırmızı, N.İ; Yigitbasi, T.; Sayin Sakul, A.; Iscan, G. Preclinical evaluation of Scorzonera sp. root extracts and major compounds against acute hepatotoxicity induced by carbon tetrachloride. Indian J. Pharm. Sci. 2017, 79. [CrossRef]

74. Erden, Y.; Kırbağ, S. Chemical and biological activities of some Scorzonera species: An in vitro study. Proc. Natl. Acad. Sci. India Sect. B Biol. Sci. 2015, 85, 319-326. [CrossRef]

75. Donia, A.E.R.M. Phytochemical and pharmacological studies on Scorzonera alexandrina Boiss. J. Saudi Chem. Soc. 2016, 20, S433-S439. [CrossRef]

76. Mukherjee, P.K. Bioassay-Guided Isolation and Evaluation of Herbal Drugs. In Quality Control and Evaluation of Herbal Drugs; Elsevier: Amsterdam, The Netherlands, 2019; pp. 515-537.

77. Zhang, W.G.; Zhao, R.; Ren, J.; Ren, L.X.; Lin, J.G.; Liu, D.L.; Wu, Y.L.; Yao, X.S. Synthesis and anti-proliferative in vitro activity of two natural dihydrostilbenes and their analogues. Arch. Pharm. 2007, 340, 244-250. [CrossRef]

78. Csupor-Löffler, B.; Hajdú, Z.; Réthy, B.; Zupkó, I.; Máthé, I.; Rédei, T.; Falkay, G.; Hohmann, J. Antiproliferative activity of Hungarian Asteraceae species against human cancer cell lines. Part II. Phyther. Res. 2009, 23, 1109-1115. [CrossRef]

79. Perveen, S.; Al-Taweel, A.M.; Yusufoglu, H.S.; Fawzy, G.A.; Foudah, A.; Abdel-Kader, M.S. Hepatoprotective and cytotoxic activities of Anvillea garcinii and isolation of four new secondary metabolites. J. Nat. Med. 2018, 72, 106-117. [CrossRef]

80. Alshehri, K.M.; Ghobashy, M.O.I. Antitumor, antimicrobial activities and phytochemicals constituent of different extracts of Pulicaria undulata (Forssk.) Oliver. grown naturally in Saudi Arabia. Int. J. Res. Pharm. Sci. 2020, 11, 4889-4901. [CrossRef]

81. Park, B.Y.; Oh, S.R.; Ahn, K.S.; Kwon, O.K.; Lee, H.K. (-)-Syringaresinol inhibits proliferation of human promyelocytic HL-60 leukemia cells via G1 arrest and apoptosis. Int. Immunopharmacol. 2008, 8, 967-973. [CrossRef]

82. Jeong, Y.H.; Chung, S.Y.; Han, A.R.; Sung, M.K.; Jang, D.S.; Lee, J.; Kwon, Y.; Lee, H.J.; Seo, E.K. P-glycoprotein inhibitory activity of two phenolic compounds, (-)-syringaresinol and tricin from Sasa borealis. Chem. Biodivers. 2007, 4, 12-16. [CrossRef] [PubMed]

83. Tasneem, S.; Liu, B.; Li, B.; Choudhary, M.I.; Wang, W. Molecular pharmacology of inflammation: Medicinal plants as antiinflammatory agents. Pharmacol. Res. 2019, 139, 126-140. [CrossRef] [PubMed]

84. Wal, P.; Wal, A.; Sharma, G.; Rai, A. Biological activities of lupeol. Syst. Rev. Pharm. 2011, 2, 96-103. [CrossRef]

85. Lucetti, D.L.; Lucetti, E.C.P.; Bandeira, M.A.M.; Veras, H.N.H.; Silva, A.H.; Leal, L.K.A.M.; Lopes, A.A.; Alves, V.C.C.; Silva, G.S.; Brito, G.A.; et al. Anti-inflammatory effects and possible mechanism of action of lupeol acetate isolated from Himatanthus drasticus (Mart.) Plumel. J. Inflamm. 2010, 7, 60. [CrossRef] [PubMed]

86. Shilpi, J.A.; Uddin, S.J. Analgesic and antipyretic natural products. In Annual Reports in Medicinal Chemistry; Elsevier: Amsterdam, The Netherlands, 2020; Volume 55, pp. 435-458.

87. Köngül-Şafak, E. Plant extracts with putative hepatoprotective activity. In Influence of Nutrients, Bioactive Compounds, and Plant Extracts in Liver Diseases; Elsevier: Amsterdam, The Netherlands, 2021; pp. 227-257.

88. Lal, M.; Chandraker, S.K.; Shukla, R. Antimicrobial properties of selected plants used in traditional Chinese medicine. In Functional and Preservative Properties of Phytochemicals; Elsevier: Amsterdam, The Netherlands, 2020; pp. 119-143.

89. Gutiérrez-del-Río, I.; Fernández, J.; Lombó, F. Plant nutraceuticals as antimicrobial agents in food preservation: Terpenoids, polyphenols and thiols. Int. J. Antimicrob. Agents 2018, 52, 309-315. [CrossRef]

90. Boussaada, O.; Saidana, D.; Chriaa, J.; Chraif, I.; Mahjoub, M.A.; Mighri, Z.; Daami, M.; Helal, A.N. Chemical composition and antimicrobial activity of volatile components of Scorzonera undulata. J. Essent. Oil Res. 2008, 20, 358-362. [CrossRef] 
91. Abdelkader, H.B.; Salah, K.B.H.; Liouane, K.; Boussaada, O.; Gafsi, K.; Mahjoub, M.A.; Aouni, M.; Hellal, A.N.; Mighri, Z. Antimicrobial activity of Rhaponticum acaule and Scorzonera undulata growing wild in Tunisia. Afr. J. Microbiol. Res. 2010, 4, 1954-1958.

92. Sweidan, A.; El-Mestrah, M.; Kanaan, H.; Dandache, I.; Merhi, F.; Chokr, A. Antibacterial and antibiofilm activities of Scorzonera mackmeliana. Pak. J. Pharm. Sci. 2020, 33, 199-206.

93. Buru, A.S.; Pichika, M.R.; Neela, V.; Mohandas, K. In vitro antibacterial effects of Cinnamomum extracts on common bacteria found in wound infections with emphasis on methicillin-resistant Staphylococcus aureus. J. Ethnopharmacol. 2014, 153, 587-595. [CrossRef]

94. Mavi, A.; Yiğit, N.; Yiğit, D.; Kandemir, A. Antioxidant and antimicrobial activity of Turkish endemic Sonchus erzincanicus extracts Turk. J. Biol. 2011, 35, 243-250. [CrossRef]

95. Mohammed, F.; Şabik, A.; Akgül, H.; Sevindik, M. Antioxidant and Antimicrobial activity of Scorzonera papposa collected from Iraq and Turkey. Kahramanmaraş Sütçü İmam Üniv. Tarım Doğa Dergisi 2020, 23, 1114-1118. [CrossRef]

96. Yadav, S.; Mishra, A.P.; Kumar, S.; Negi, A.; Maurya, V.K. Herbal wound healing agents. In Preparation of Phytopharmaceuticals for the Management of Disorders; Elsevier: Amsterdam, The Netherlands, 2021; pp. 169-184.

97. Khuda-Bukhsh, A.R.; Saha, S.K.; Das, S.; Saha, S.S. Molecular approaches toward targeted cancer therapy with some food plant products: On the role of antioxidants and immune microenvironment. In Cancer; Elsevier: Amsterdam, The Netherlands, 2021; pp. 191-202.

98. Olszowy, M. What is responsible for antioxidant properties of polyphenolic compounds from plants? Plant Physiol. Biochem. 2019, 144, 135-143. [CrossRef] [PubMed]

99. Petrovic, M.; Suznjevic, D.; Pastor, F.; Veljovic, M.; Pezo, L.; Antic, M.; Gorjanovic, S. Antioxidant capacity determination of complex samples and individual phenolics-multilateral approach. Comb. Chem. High Throughput Screen. 2016, 19, 58-65. [CrossRef] [PubMed] 